\title{
LIE ALGEBRAS AND TORSION GROUPS WITH IDENTITY
}

\author{
E. ZELMANOV \\ To my teacher Leonid A. Bokut on his 80th birthday.
}

\begin{abstract}
We prove that a finitely generated Lie algebra $L$ such that (i) every commutator in generators is ad-nilpotent, and (ii) $L$ satisfies a polynomial identity, is nilpotent. As a corollary we get that a finitely generated residually- $p$ torsion group whose pro- $p$ completion satisfies a pro- $p$ identity is finite.
\end{abstract}

\section{INTRODUCTION}

In 1941 A.G Kurosh formulated a Burnside-type problem for algebras Kur. Let $A$ be an associative algebra over a field $F$. An element $a \in A$ is said to be nilpotent if $a^{n(a)}=0$ for some $n(a) \geq 1$. An algebra $A$ is said to be nil if every element of $A$ is nilpotent.

The Kurosh Problem: Is it true that a finitely generated nil algebra is nilpotent?

Examples by E. S. Golod Gol (see also the far reaching examples from [LS]) showed that this is not always the case. However the Kurosh Problem has positive solution in the class of algebras satisfying a polynomial identity (PI-algebras).

Let $f\left(x_{1}, x_{2} \cdots, x_{m}\right)$ be a nonzero element of the free associative $F$-algebra. We say that an algebra $A$ satisfies the polynomial identity $f=0$ if $f\left(a_{1}, a_{2}, \cdots, a_{m}\right)=0$ for arbitrary elements $a_{1}, a_{2}, \cdots, a_{m} \in A$.

2010 Mathematics Subject Classification. Primary: 20E18, 20F50, 20F40, 16R99.

Key words and phrases. the Burnside problem, pro-p groups, PI-algebras, Lie algebras. 
One of the high points of the theory of PI-algebras was the solution of the Kurosh Problem (I. Kaplansky Kap, J. Levitzki [Lev], A. I. Shirshov [Shi]) in the following form:

Let $A$ be an associative algebra generated by elements $a_{1}, \cdots, a_{m}$. Let $S$ be the multiplicative semigroup generated by the elements $a_{1}, \cdots, a_{m}$. Suppose that an arbitrary element of $S$ is nilpotent. Then the algebra $A$ is nilpotent.

Now let $L$ be a Lie algebra over a field $F$. As above, for a nonzero element $f\left(x_{1}, x_{2}, \cdots, x_{m}\right)$ of the free Lie algebra we say that $L$ satisfies the identity $f=0$ if $f\left(a_{1}, a_{2}, \cdots, a_{m}\right)=0$ for arbitrary elements $a_{1}, a_{2}, \cdots, a_{m} \in A$, see $\mathrm{Bah}$.

An element $a \in L$ is said to be ad-nilpotent if the linear operator

$$
\operatorname{ad}(a): L \rightarrow L, x \rightarrow[x, a]
$$

is nilpotent.

A subset $S \subset L$ is called a Lie set if, for arbitrary elements $a, b \in S$, we have $[a, b] \in S$. For a subset $X \subset L$, the Lie set generated by $X$ is the smallest Lie set $S\langle X\rangle$ containing $X$. It consists of $X$ and of all iterated commutators in elements from $X$.

Theorem 1.1. Let $L$ be a Lie algebra satisfying a polynomial identity and generated by elements $a_{1}, \cdots, a_{m}$. If an arbitrary element $s \in$ $S\left\langle a_{1}, \cdots, a_{m}\right\rangle$ is ad-nilpotent then the Lie algebra $L$ is nilpotent.

This theorem has implications in group theory:

Let $p$ be a prime number. A group $G$ is said to be residually-p if there exists a family of homomorphisms $\phi_{i}: G \rightarrow G_{i}$ into finite $p$-groups $G_{i}$ such that $\bigcap_{i} \operatorname{Ker}\left(\phi_{i}\right)=(1)$.

Let $\mathbb{Z}_{p}$ be the field of order $p$. Consider the group algebra $\left(\mathbb{Z}_{p}\right)[G]$ and its fundamental ideal $w$ spanned by all elements $1-g, g \in$ $G$. It is easy to see that the group $G$ is residually- $p$ if and only if $\bigcap_{i \geq 1} w^{i}=(0)$. The Zassenhaus filtration is defined as

$$
G=G_{1}>G_{2}>\cdots
$$


where $G_{i}=\left\{g \in G \mid 1-g \in w^{i}\right\}$. Then $\left[G_{i}, G_{j}\right] \subseteq G_{i+j}$ and each factor $G_{i} / G_{i+1}$ is an elementary abelian $p$-group. Hence

$$
L_{p}(G)=\bigoplus_{i \geq 1} G_{i} / G_{i+1}
$$

is a Lie algebra over $\mathbb{Z}_{p}$.

Theorem 1.2. Let $G$ be a residually-p finitely generated torsion group such that the Lie algebra $L_{p}(G)$ satisfies a polynomial identity. Then $G$ is a finite group.

Let $g\left(x_{1}, x_{2}, \cdots, x_{m}\right)$ be a nonidentical element of the free pro- $p$ group (see [Ser], DdSMS]) on the set of free generators $x_{1}, x_{2}, \cdots, x_{m}$. We say that a pro- $p$ group $G$ satisfies the identity $g=1$ if $g\left(a_{1}, a_{2}, \cdots, a_{m}\right)=1$ for arbitrary elements $a_{1}, a_{2}, \cdots, a_{m} \in G$.

Theorem 1.3. Let $G$ be a residually-p finitely generated torsion group such that its pro-p completion $G_{\hat{p}}$ satisfies a nontrivial identity. Then $G$ is a finite group.

Remark: the examples of infinite residually- $p$ groups due to E.S. Golod Gol, R. I. Grigorchuk Gri1, and N. Gupta-S. Sidki GS] are finitely generated and torsion.

The results above significantly extend the positive solution of the Restricted Burnside Problem [Zel4, Zel5] and the work of [Zel6] on compact torsion groups. They were announced in [Zel8, Zel7] but no detailed proof followed. Meanwhile they were used in numerous papers. Therefore I feel compelled to present a detailed proof.

The proof essentially uses the ideas and techniques from [Zel4, Zel5].

\section{Acknowledgments}

The author is grateful to the referees of this paper and to A. Fernandez Lopez for numerous valuable comments.

\section{The CASE OF ZERO CHARACTERISTIC}

In this section we assume that $\operatorname{char} F=0$. This assumption allows us to avoid major difficulties but also miss major applications. 
The following lemma is due to A. I. Kostrikin [Kos1; Kos2, Lemma 2.1.1].

Kostrikin Lemma. Let $L$ be a Lie algebra, $a \in L, \operatorname{ad}(a)^{n}=0$. If $4 \leq n<\operatorname{char} F$ (here zero characteristic is viewed as $\infty$ ), then

$$
\operatorname{ad}\left(b \operatorname{ad}(a)^{n-1}\right)^{n-1}=0
$$

for an arbitrary element $b \in L$.

Choose a nonzero element $s \in S=S\left\langle a_{1}, \cdots, a_{m}\right\rangle$. The element $s$ is ad-nilpotent. Repeatedly using the Kostrikin lemma we can assume that $\operatorname{ad}(s)^{3}=0$.

Recall that a linear algebra over a field $F$ of characteristic $\neq 2$ is called a Jordan algebra if it satisfies the identities

$$
\begin{aligned}
& (\mathrm{J} 1) x \circ y=y \circ x \\
& (\mathrm{~J} 2)\left(x^{2} \circ y\right) \circ x=x^{2} \circ(y \circ x) .
\end{aligned}
$$

If $\mathrm{A}$ is an associative algebra then $A^{(+)}=\left\{A, a \circ b=\frac{1}{2}(a b+b a)\right\}$ is a Jordan algebra. For more information on Jordan algebras see Jac1, ZSSS, McC2].

We will use a construction of a Jordan algebra from [FLGGL which is a refined version of the Tits-Kantor-Koecher construction [Tit1, Tit2, Kan, Koe.

Let $L$ be a Lie algebra over a field of characteristic $\neq 2,3$. Let $s \in L$, $\operatorname{ad}(s)^{3}=0$. Define a new operation $a \circ b=[a,[s, b]], a, b \in L$. Then the vector space $K=\left\{a \in L \mid a \operatorname{ad}(s)^{2}=0\right\}$ is an ideal of the algebra $(L, \circ)$.

Theorem 2.1 ([FLGGL]). The factor algebra $(L, \circ) / K$ is a Jordan algebra.

For a set $X=\left\{x_{1}, x_{2}, \cdots\right\}$, let $F J\langle X\rangle$ denote the free Jordan algebra (see [Jac1, ZSSS, McC2]). Consider also the free associative algebra $F\langle X\rangle$. Let $\phi$ be the homomorphism $\phi: F J\langle X\rangle \rightarrow F\langle X\rangle^{(+)}$, $x \rightarrow x, x \in X$. An element lying in the kernal ker $\phi$, is called an $S$-identity. A Jordan algebra $J$ is said to be $P I$ if there exists an 
element $f\left(x_{1}, \cdots, x_{n}\right) \in F J\langle X\rangle$ that is not an $S$-identity such that $f\left(a_{1}, \cdots, a_{n}\right)=0$ for all elements $a_{1}, \cdots, a_{n} \in J$.

For elements $x, y, z$ of a Jordan algebra $J$, define their triple product $\{x, y, z\}=(x y) z+x(y z)-y(x z)$. An element $a \in J$ is called an

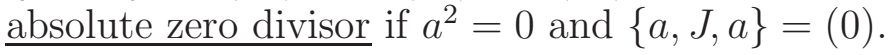

A Jordan algebra that does not contain nonzero absolute zero divisors is called nondegenerate. The smallest ideal $M c(J)$ such that the factor algebra $J / M c(J)$ is nondegenerate is called the McCrimmon radical of $J$.

Lemma 2.2. Let $J$ be a Jordan algebra with PI such that every element of $J$ is a sum of nilpotent elements. Then $J=M c(J)$.

Proof. Let $J \neq M c(J)$. Then without loss of generality we will assume that the algebra $J$ is nondegenerate. Moreover, since a nondegenerate Jordan algebra is a subdirect product of prime nondegenerate Jordan algebras (see [Zel3]), we will assume that the algebra $J$ is prime and nondegenerate.

In [Zel2 it was shown that a prime nondegenerate PI-algebra $J$ has nonzero center

$$
Z(J)=\{z \in J \mid(z a) b=z(a b) \text { for arbitrary elements } a, b \in J\}
$$

and the ring of fractions $\widetilde{J}=(Z(J) \backslash\{0\})^{-1} J$ is either a simple finite dimensional algebra over the field $\widetilde{Z}=(Z(J) \backslash\{0\})^{-1} Z(J)$ or else an algebra of a symmetric nondegenerate bilinear form. In both cases the algebra $\widetilde{J}$ has a nonzero linear trace $t: \widetilde{J} \rightarrow \widetilde{Z}$ such that the trace of a nilpotent element is zero. Since every element of $\widetilde{J}$ is a sum of nilpotent elements it follows that $t(\widetilde{J})=(0)$, a contradiction that finishes the proof of the lemma.

Lemma 2.3. The Jordan algebra $J=(L, \circ) / K$ is $M c$ Crimmon radical, i.e. $J=M c(J)$.

Proof. By our assumption, the Lie algebra $L$ satisfies a nontrivial polynomial identity. Passing to the full linearization of this identity (see [ZSSS] ) we can assume that the identity looks like

$$
\sum_{\sigma \in S_{n}} \alpha_{\sigma} x_{0} \operatorname{ad}\left(x_{\sigma(1)}\right) \cdots \operatorname{ad}\left(x_{\sigma(n)}\right)=0,
$$


where not all coefficients $\alpha_{\sigma} \in F$ are equal to 0 . This implies that

$$
\sum_{\sigma \in S_{n}} \alpha_{\sigma} a_{0} R\left(a_{\sigma(1)}\right) \cdots R\left(a_{\sigma(n)}\right)=0
$$

for arbitrary elements $a_{0}, a_{1} \cdot \cdots, a_{n} \in J$, where $R(a): x \rightarrow x a$ denotes the multiplication operator in $J$.

It is easy to see that the element $\sum_{\sigma \in S_{n}} \alpha_{\sigma} a_{0} R\left(a_{\sigma(1)}\right) \cdots R\left(a_{\sigma(n)}\right)$ is not an $S$-identity. Hence $J$ is a PI-algebra.

The Lie algebra $L$ is spanned by the Lie set $S=S\left\langle a_{1}, \cdots a_{m}\right\rangle$. For an arbitrary element $a \in S$ let $\bar{a}=a+K$ be its image in the Jordan algebra $J$. The $\mathrm{t}^{t h}$ power of $\bar{a}$ in $J$ is $a \operatorname{ad}([s, a])^{t-1}+K$, which implies that the element $\bar{a}$ is nilpotent. By Lemma $2.2 J=M c(J)$, which finishes the proof of Lemma 2.3.

Following A. I. Kostrikin Kos1, Kos3, we call an element $a$ of a Lie algebra $L$ a sandwich if (i) $\operatorname{ad}(a)^{2}=0$ and (ii) $\operatorname{ad}(a) \operatorname{ad}(b) \operatorname{ad}(a)=0$ for an arbitrary element $b \in L$.

If $\operatorname{char} F \neq 2$ then (i) implies (ii).

If $a$ is a nonzero absolute zero divisor of the Jordan algebra $J$ and char $F \neq 2,3$ then for the nonzero element $b=a \operatorname{ad}(s)^{2}$ we have $\operatorname{ad}(b)^{2}=\operatorname{ad}(s)^{2} \operatorname{ad}(a)^{2} \operatorname{ad}(s)^{2}$. Hence $L \operatorname{ad}(b)^{2} \subseteq\{a, J, a\} \operatorname{ad}(s)^{2}=$ $(0)$. Hence, $b$ is a sandwich of the Lie algebra $L$.

To summarize, we showed that if $L=\left\langle a_{1}, \cdots, a_{m}\right\rangle$ is a nonzero Lie algebra over a field $F$ of zero characteristic, every element of the Lie set $S=S\left\langle a_{1}, \cdots, a_{m}\right\rangle$ is ad-nilpotent, and if $L$ satisfies a nontrivial polynomial identity, then $L$ contains a nonzero sandwich.

Lemma 2.4. Let $L=\left\langle a_{1}, \cdots, a_{m}\right\rangle$ be a finitely generated Lie algebra such that an arbitrary element of the Lie set $S\left\langle a_{1}, \cdots, a_{m}\right\rangle$ is adnilpotent. Let I be an ideal of $L$ of finite codimension. Then I is finitely generated as a Lie algebra.

Proof. The finite dimensional Lie algebra $L / I$ is spanned by a Lie set for which every element in the set is ad-nilpotent. By the Engel-Jacobson theorem Jac2 the Lie algebra $L / I$ is nilpotent. In other words, there exists $k \geq 1$ such that $L^{k} \subseteq I$. 
Suppose that every commutator $\rho$ in $a_{1} \cdots, a_{m}$ of length $<k$ is adnilpotent of degree at most $t$, i.e. $\operatorname{ad}(\rho)^{t}=0$. Let $N=\mathrm{ktm}^{k}$. In [Zel7, Lemma 2.5] it is shown that every product $\operatorname{ad}\left(a_{i_{1}}\right) \cdots \operatorname{ad}\left(a_{i_{N}}\right)$, $1 \leq i_{1}, \cdots, i_{N} \leq m$, can be represented as

$$
\operatorname{ad}\left(a_{i_{1}}\right) \cdots \operatorname{ad}\left(a_{i_{N}}\right)=\sum_{j} v_{j} \operatorname{ad}\left(\rho_{j}\right)
$$

where the $v_{j}$ 's are (possibly empty) products of the $a d\left(a_{i}\right)$ 's and the $\rho_{j}$ 's are commutators in $a_{1}, \cdots, a_{m}$ of length $\geq \mathrm{k}$. Each summand on the right hand side has the same degree in each $a_{i}$ as the left hand side.

It follows now that the algebra $L^{k}$ is generated by commutators $\rho$ in $a_{1}, \cdots, a_{m}$ such that $k \leq \operatorname{length}(\rho)<2 N$.

We have $\operatorname{dim}_{F}\left(L / L^{k}\right)<\infty$. Let $b_{1}, \cdots, b_{r} \in I$ be a basis of $I$ modulo $L^{k}$. Now the algebra $I$ is generated by $b_{1}, \cdots, b_{r}$ and by all commutators $\rho$ in $a_{1}, \cdots, a_{m}$ such that $k \leq \operatorname{length}(\rho)<2 N$, which proves the lemma.

Recall that an algebra $L$ is called just infinite if it is infinite dimensional but every nonzero ideal of $L$ is of finite codimension.

Lemma 2.5. Let $L$ be an infinite dimensional Lie algebra generated by elements $a_{1}, \cdots, a_{m}$ such that an arbitrary element from $S\left\langle a_{1}, \cdots, a_{m}\right\rangle$ is ad-nilpotent. Then $L$ has a just infinite homomorphic image.

Proof. Let $I_{1} \subseteq I_{2} \subseteq$. . be an ascending chain of ideals of infinite codimension. We claim that the union $I=\bigcup_{i} I_{i}$ also has infinite codimension. Indeed, if $\operatorname{dim}_{F}(L / I)<\infty$ then by Lemma 2.4 the ideal $I$ is generated by a finite collection of elements, hence $I$ is equal to one of the terms in the ascending chain, a contradiction.

By Zorn's Lemma the algebra $L$ has a maximal ideal $J$ of infinite codimension. The factor algebra $L / J$ is just infinite, which proves the lemma.

Now we are ready to finish the proof of Theorem 1.1 in the case of $\operatorname{char} F=0$. 
Let $L$ be a Lie algebra satisfying the assumptions of the theorem. In view of Lemma 2.5 without loss of generality we will assume the algebra $L$ to be just infinite.

We proved that $L$ contains a nonzero sandwich. Recall that an algebra is called locally nilpotent if every finitely generated subalgebra is nilpotent. A. N. Grishkov Gri2 proved that in a Lie algebra over a field of zero characteristic, an arbitrary sandwich generates a locally nilpotent ideal. Since the Lie algebra $L$ is just infinite it follows that $L$ contains a locally nilpotent ideal $I$ of finite codimension. By Lemma 2.2 the algebra $I$ is finitely generated, hence nilpotent and finite dimensional. This contradicts the assumption that the algebra $L$ is infinite dimensional and proves the theorem.

\section{Divided POLYNOMials}

The main Theorem 1.1 is valid for Lie algebras over an arbitrary ground field $F$. The applications to Theorems [1.2, [1.3 use only the case when the ground field $F$ is finite.

We will show that without loss of generality, we can assume that the field $F$ is infinite. Indeed, let $F^{\prime}$ be an infinite field extension of $F$. The Lie algebra $L^{\prime}=L \otimes_{F} F^{\prime}$ is generated by the same elements $a_{1}, \cdots, a_{m}$ as $L$ and an arbitrary element $s \in S\left\langle a_{1}, \cdots, a_{m}\right\rangle$ is ad-nilpotent in $L^{\prime}$. Since the Lie algebra $L$ satisfies a polynomial identity, it satisfies a nontrivial multilinear identity $f\left(x_{1}, \cdots, x_{n}\right)=0$ (see [Bah]). Then the Lie algebra $L^{\prime}$ also satisfies the identity $f=0$.

From now on we assume that $\operatorname{char} F=p>0$ and the field $F$ is infinite. Let $L\langle X\rangle$ be the free Lie $F$-algebra on the set of free generators $X=\left\{x_{1}, \cdots, x_{m}\right\}$ in the variety of algebras satisfying the identity $f=0$ (see [Bah]). Let $P$ be the set of all commutators in $X$ and let $n: P \rightarrow N$ be a function. Let $J$ be the ideal of $L\langle X\rangle$ generated by $\bigcup_{\rho \in P} L\langle X\rangle \operatorname{ad}(\rho)^{n(\rho)}$.

Our aim is to show that the algebra $L^{\prime}=L\langle X\rangle / J$ is nilpotent. Suppose that this is not true. Letting $\operatorname{deg}\left(x_{i}\right)=1,1 \leq i \leq m$, we define a gradation of $L^{\prime}$ by positive integers. 
We say that a graded infinite dimensional algebra is graded just infinite if every nonzero graded ideal of it is of finite codimension.

Lemma 3.1. The algebra $L^{\prime}$ has a graded just infinite homomorphic image $L$.

The proof follows the proof of Lemma 2.5 (verbatim).

Consider the adjoint embedding $L \rightarrow \operatorname{End}_{F}(L), a \rightarrow \operatorname{ad}(a)$. Let $A^{\prime}$ be the associative subalgebra of $\operatorname{End}_{F}(L)$ generated by the image of $L$. The algebra $A^{\prime}$ is graded and we assume that $L \subseteq A^{\prime(-)}$. Let $I$ be a maximal graded ideal of the algebra $A^{\prime}$ such that $L \cap I=(0), A=A^{\prime} / I$, $L \subseteq A^{(-)}$. If $J$ is a nonzero graded ideal of the algebra $A$ then the ideal $J \cap L$ has finite codimension in $L$. From the Poincare-Birkhoff-Witt theorem it follows that the factor algebra $A / J$ is nilpotent and finite dimensional. We have proved that the algebra $A$ is graded just infinite.

To summarize, we assume that

(1) the graded Lie algebra $L$ is generated by elements $s_{1}, \cdots, s_{m}$ of degree 1; every element from the Lie set $S=S\left\langle s_{1}, \cdots, s_{m}\right\rangle$ is ad-nilpotent;

(2) $L$ satisfies a polynomial identity;

(3) $L$ is graded just infinite.

We fix also a graded just infinite associative enveloping algebra $A$ of $L$. The algebra $A$ is a homomorphic image of the subalgebra $\langle\operatorname{ad}(L)\rangle \subseteq$ $\operatorname{End}_{F}(L)$

For elements $a_{1}, \cdots, a_{k} \in L$ let $\left[a_{1}, a_{2}, \cdots, a_{k}\right]$ denote their leftnormed commutator by $\left.\left[\cdots\left[a_{1}, a_{2}\right], a_{3}\right], \cdots, a_{k}\right]$ We also denote $[a, \underbrace{b, b \cdots, b}_{\mathrm{k}}]=\left[a b^{k}\right]$.

Lemma 3.2. Let $I$ be an ideal of $L, s \in S, k \geq 2,\left[I s^{k}\right]=(0)$. Suppose that the Lie algebra $L$ satisfies an identity of degree $n$. Then the subalgebra $\left[\mathrm{Is}^{k-1}\right]$ satisfies an identity of degree $<n$.

Proof. Let $L$ satisfy an identity

$$
\sum_{\sigma \in S_{n-1}} \alpha_{\sigma}\left[x_{0}, x_{\sigma(1)}, \cdots, x_{\sigma(n-1)}\right]=0
$$


where $\alpha_{\sigma} \in F, \alpha_{1}=1$. For the variables $x_{0}, x_{1}, \cdots, x_{n-1}$ choose values $x_{0}=s, x_{1}=a \in I, x_{i}=a_{i} \in\left[I s^{k-1}\right], 2 \leq i \leq n-1$. Then

$$
\sum_{\sigma \in H} \alpha_{\sigma}\left[[s, a], a_{\sigma(2)}, \cdots, a_{\sigma(n-1)}\right]=0
$$

where $\sigma$ runs over the stabilizer $H$ of 1 in $S_{n-1}$. It follows now that the Lie algebra $\left[I s^{k-1}\right]$ satisfies the identity

$$
\sum_{\sigma \in H} \alpha_{\sigma}\left[x_{0}, x_{\sigma(2)}, \cdots, x_{\sigma(n-1)}\right]=0
$$

of degree $n-1$. This finishes the proof of the lemma.

Lemma 3.3. Let $I$ be an ideal of $L, s \in L,\left[I s^{k}\right]=(0), k \geq 2$. Then for any integer $t \geq 2$ and any elements $a_{1}, \cdots, a_{N} \in I, N=k t-1$, we can write the operator ad $\left(\left[a_{1} s^{k-1}\right]\right) \cdots a d\left(\left[a_{N} s^{k-1}\right]\right)$ as a linear combination of operators of the type

$$
P^{\prime} a d(s)^{k-1} \prod_{j=0}^{t-2}\left(a d\left(a_{i+j}\right) a d(s)^{k-1}\right) P^{\prime \prime}
$$

where $P^{\prime}, P^{\prime \prime}$ are products of ad $\left(a_{1}\right), \cdots$, ad $\left(a_{n}\right)$, ad $(s)$, which may be empty.

Proof. By the Jacobi identity

$$
\begin{gathered}
\operatorname{ad}\left(\left[a_{1} s^{k-1}\right]\right) \cdots \operatorname{ad}\left(\left[a_{N} s^{k-1}\right]\right. \\
=\sum \pm \operatorname{ad}(s)^{j_{0}} \operatorname{ad}\left(a_{1}\right) \operatorname{ad}(s)^{j_{1}} \cdots \operatorname{ad}(s)^{j_{N-1}} \operatorname{ad}\left(a_{N}\right) \operatorname{ad}(s)^{j_{N}},
\end{gathered}
$$

and in each summand $0 \leq j_{0}, j_{1}, \cdots, j_{N} \leq k-1, j_{0}+\cdots+j_{N}=(k-1) N$. If, in each segment $j_{\mu}, j_{\mu+1} \cdots, j_{\mu+t-1}$ of length $t$, at least one term is $\leq k-2$, then $j_{\mu}+\cdots \jmath_{\mu+t-1} \leq(k-1) t-1$. Summing all $k$ segments we get $j_{0}+\cdots+\jmath_{N} \leq k((k-1) t-1)<(k-1)(k t-1)$, a contradiction that proves the lemma.

Lemma 3.4. There exist elements $c_{1}, \cdots, c_{r} \in S$ and integers $m \geq$ $1, N \geq 1$ such that

(1) $\left[L^{i}, c_{1}, c_{2}, \cdots, c_{r}\right] \neq(0)$ for arbitrary $i \geq 1$,

(2) $\left[L^{m}, c_{1}, c_{2}, \cdots, c_{r}, c_{i}\right]=(0)$ for $1 \leq i \leq r$,

(3) for arbitrary elements $a_{1}, \cdots, a_{N} \in\left[L^{m}, c_{1}, \cdots, c_{r}\right]$ we have $\operatorname{ad}\left(a_{1}\right) \cdots \operatorname{ad}\left(a_{N}\right)=0$. 
Proof. Choose a nonzero element $s \in S$. For an arbitrary $i \geq 1$ choose a minimal integer $k(i) \geq 1$ such that $\left[L^{i} s^{k(i)}\right]=(0)$. Since the Lie algebra $L$ is graded just infinite it follows that each power of $L$ has zero centralizer. Hence $k(i) \geq 2$. We have $k(1) \geq k(2) \geq \cdots$. There exists a sufficiently large integer $m_{1}$ such that

$$
k_{1}:=k\left(m_{1}\right)=k\left(m_{1}+1\right)=\cdots .
$$

In other words, $\left[L^{m_{1}} s^{k_{1}}\right]=(0),\left[L^{i} s^{k_{1}-1}\right] \neq(0)$ for any $i \geq 1$.

Now suppose that we have found $l$ elements $s_{1}=s, s_{2}, \cdots, s_{l} \in S$ and $2 l$ integers $k_{1}, \cdots, k_{l} \geq 2 ; 1 \leq m_{1} \leq m_{2} \leq \cdots \leq m_{l}$ with the following properties:

(1) $s_{i} \in\left[L^{m_{i-1}} s_{1}^{k_{1}-1} \cdots s_{i-1}^{k_{i-1}-1}\right], 2 \leq i \leq l$,

(2) $\left[L^{m_{i}} s_{1}^{k_{1}-1} \cdots s_{i-1}^{k_{i-1}-1} s_{i}^{k_{i}}\right]=(0), 1 \leq i \leq l$,

(3) for an arbitrary $i \geq 1$ we have $\left[L^{i} s_{1}^{k_{1}-1} \cdots s_{l}^{k_{l}-1}\right] \neq(0)$.

Claim 3.5. For arbitrary $1 \leq i, j \leq l$ we have $\left[s_{i}, s_{j}\right]=0$.

Proof. Indeed, let $i<j$. Then $s_{j} \in\left[L^{m_{j-1}} s_{1}^{k_{1}-1} \cdots s_{j-1}^{k_{j-1}-1}\right]$. We will show that

$$
\left[L^{m_{j-1}} s_{1}^{k_{1}-1} \cdots s_{j-1}^{k_{j-1}-1} s_{i}\right]=(0) .
$$

By the inductive assumption on $i+j$ the element $s_{i}$ commutes with $s_{i+1}, \cdots, s_{j-1}$. Hence

$$
\left[L^{m_{j-1}} s_{1}^{k_{1}-1} \cdots s_{j-1}^{k_{j-1}-1} s_{i}\right]=\left[L^{m_{j-1}} s_{1}^{k_{1}-1} \cdots s_{i}^{k_{i}} \cdots\right]=0,
$$

which proves the claim.

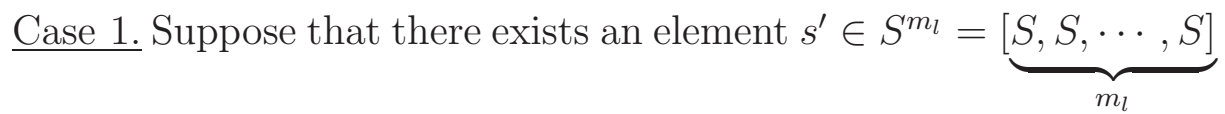
such that

$$
\left[\left[L^{i} s_{1}^{k_{1}-1} \cdots s_{l}^{k_{l}-1}\right],\left[s^{\prime} s_{1}^{k_{1}-1} \cdots s_{l}^{k_{l}-1}\right]\right] \neq(0)
$$

for any $i \geq 1$. Denote $s_{l+1}=\left[s^{\prime} s_{1}^{k_{1}-1} \cdots s_{l}^{k_{l}-1}\right]$. As above we find integers $m_{l+1} \geq m_{l}$ and $k_{l+1} \geq 2$ such that

$$
\begin{gathered}
{\left[\left[L^{m_{l+1}} s_{1}^{k_{1}-1} \cdots s_{l}^{k_{l}-1}\right] s_{l+1}^{k_{l+1}}\right]=(0),} \\
{\left[L^{i} s_{1}^{k_{1}-1} \cdots s_{l}^{k_{l}-1} s_{l+1}^{k_{l+1}-1}\right] \neq(0)}
\end{gathered}
$$

for any $i \geq 1$. The elements $s_{1}, \cdots, s_{l+1}$ and the integers $m_{1}, \cdots, m_{l+1}$; $k_{1}, \cdots k_{l+1}$ satisfy the conditions 1), 2), and 3) above. 
$\underline{\text { Case } 2}$ Now suppose that for an arbitrary element $s^{\prime} \in S^{m_{l}}$ there exists an integer $i\left(s^{\prime}\right) \geq 1$ such that

$$
\left[\left[L^{i\left(s^{\prime}\right)} s_{1}^{k_{1}-1} \cdots s_{l}^{k_{l}-1}\right],\left[s^{\prime} s_{1}^{k_{1}-1} \cdots s_{l}^{k_{l}-1}\right]\right]=(0) .
$$

Since $S^{m_{l}}$ spans $L^{m_{l}}$ it follows that for an arbitrary element $a \in$ $\left[L^{m_{l}} s_{1}^{k_{1}-1} \cdots s_{l}^{k_{l}-1}\right]$ there exists $i(a) \geq 1$ such that

$$
\left[\left[L^{i(a)} s_{1}^{k_{1}-1} \cdots s_{l}^{k_{l}-1}\right], a\right]=(0) .
$$

Let $t=2 k_{1} \cdots k_{l}-1$. Choose $2 t-1$ elements $a_{1}, \cdots, a_{2 t-1} \in$ $\left[L^{m_{l}} s_{1}^{k_{1}-1} \cdots s_{l}^{k_{l}-1}\right]$. Let $q=\max \left\{i\left(a_{\mu}\right), 1 \leq \mu \leq 2 t-1, m_{l}\right\}$.

Our immediate aim will be to show that

$$
\left[L^{q}, a_{1}, \cdots, a_{t}\right]=(0) .
$$

Denote $t_{j}=2 k_{j+1} \cdots k_{l}-1$, so $t_{0}=t$. We let $t_{l}=1$. From Claim 3.5 it follows that for any $1 \leq i \leq l, L_{j}:=\left[L^{q} s_{1}^{k_{1}-1} \cdots s_{j}^{k_{j}-1}\right]$ is a subalgebra of $L$. Let $L_{0}=L^{q}$.

Claim 3.6. For $t_{j}$ arbitrary elements $b_{1}, \cdots, b_{t_{j}} \in\left\{a_{1}, \cdots, a_{t}\right\}$ we have

$$
\left[L_{j}, b_{1}, \cdots, b_{t_{j}}\right]=(0) \text {. }
$$

Proof. To prove the claim, we will use reverse induction on $j=0, \cdots, l$. For $j=l$ we have $t_{l}=1$ and $\left[L_{l}, a_{i}\right]=\left[L^{q} s_{1}^{k_{1}-1} \cdots s_{l}^{k_{l}-1}, a_{i}\right]=(0)$ by the choice of $q$. Now suppose that the assertion is true for $j$, $1 \leq j \leq l$. We have $t_{j-1}=k_{j}\left(t_{j}+1\right)-1$. By Claim 3.5 an arbitrary element $a \in\left[L^{q} s_{1}^{k_{1}-1} \cdots s_{l}^{k_{l}-1}\right]$ can be represented as $a=\left[a^{\prime} s_{j}^{k_{j}-1}\right]$, where $a^{\prime} \in\left[L^{q} s_{1}^{k_{1}-1} \cdots s_{j-1}^{k_{1}-1}\right]=L_{j-1}$. Let $b_{\mu}=\left[b_{\mu}^{\prime} s_{j}^{k_{j}-1}\right], b_{\mu}^{\prime} \in L_{j-1}$. We apply Lemma 3.3 to the algebra $L_{j-1}+F s_{j}$ and its ideal $L_{j-1}$. By Lemma $3.3 \mathrm{ad}\left(b_{1}\right) \cdots \operatorname{ad}\left(b_{t_{j-1}}\right)$ is a linear combination of operators $P^{\prime} \operatorname{ad}\left(s_{j}\right)^{k_{j}-1}\left(\prod_{\mu=0}^{t_{j}-1} \operatorname{ad}\left(b_{i+\mu}^{\prime} \operatorname{ad}\left(s_{j}\right)^{k_{j}-1}\right)\right) P^{\prime \prime}$. By the induction assumption

$$
\begin{gathered}
L_{j-1} P^{\prime} \operatorname{ad}\left(s_{j}\right)^{k_{j}-1}\left(\prod_{\mu=0}^{t_{j}-1} \operatorname{ad}\left(b_{i+\mu}^{\prime}\right) \operatorname{ad}\left(s_{j}\right)^{k_{j}-1}\right) \\
\subseteq L_{j-1} \operatorname{ad}\left(s_{j}\right)^{k_{j}-1}\left(\prod_{\mu=0}^{t_{j}-1} \operatorname{ad}\left(b_{i+\mu}^{\prime}\right) \operatorname{ad}\left(s_{j}\right)^{k_{j}-1}\right) \\
=L_{j} \prod_{\mu=0}^{t-1} \operatorname{ad}\left(b_{i+\mu}\right),
\end{gathered}
$$

which finishes the proof of Claim 3.6 . 
In particular, for $j=0$ we have

$$
\left[L^{q}, a_{1}, a_{2}, \cdots, a_{t}\right]=(0) .
$$

Now,

$$
\left[L^{q},\left[L, a_{1}, a_{2}, \cdots, a_{2 t-1}\right]\right] \subseteq \sum\left[L^{q}, a_{i_{1}}, \cdots, a_{i_{\mu}}, L, a_{j_{1}}, \cdots, a_{j_{\nu}}\right],
$$

where in each summand $\mu+\nu=2 t-1$. If $\mu \geq t$ then

$$
\left.\left[L^{q}, a_{i_{1}}, \cdots, a_{i_{\mu}}\right]=(0)\right] \text {. }
$$

If $\nu \geq t$ then

$$
\left[L^{q}, a_{i}, \cdots, a_{i_{\mu}}, L, a_{j_{1}} \cdots, a_{j \nu}\right] \subseteq\left[L^{q}, a_{j_{1}}, \cdots, a_{j_{\nu}}\right]=(0) .
$$

Since the power $L^{q}$ has zero centralizer it follows that

$$
\left[L, a_{1}, a_{2}, \cdots, a_{2 t-1}\right]=(0) .
$$

We showed that in case 2 , the elements

$$
c_{1}, \cdots, c_{r}=\underbrace{s_{1}, \cdots, s_{1}}_{k_{1}-1}, \underbrace{s_{2}, \cdots, s_{2}}_{k_{2}-1}, \cdots, \underbrace{s_{l}, \cdots, s_{l}}_{k_{l}-1}
$$

and the integers $m=m_{l}, N=4 k_{1} \cdots k_{l}-3$ satisfy the conditions of the lemma.

Let the algebra $L$ satisfy an identity of degree $n$. We will show that enlarging the system $s_{1}, \cdots, s_{l} ; k_{1} \cdots, k_{l} \geq 2 ; 1 \leq m_{1} \leq \cdots \leq m_{l}$ we will encounter case 2 in $\leq n-2$ steps.

The subalgebra $I_{i}=\left[L^{m_{i}} s_{1}^{k_{1}-1} \cdots, s_{i-1}^{k_{i-1}-1}\right]$ is an ideal of

$$
\left[L^{m_{i-1}} s_{1}^{k_{1}-1} \cdots, s_{i-1}^{k_{i-1}-1}\right]
$$

and $\left[I_{i} s_{i}^{k_{i}}\right]=(0)$. If $\left[L^{m_{i-1}} s_{1}^{k_{1}-1} \cdots s_{i-1}^{k_{i-1}-1}\right]$ satisfies an identity of degree $n_{i-1}$ then by Lemma 3.2 the algebra $\left[L^{m_{i}} s_{1}^{k_{1}-1} \cdots, s_{i-1}^{k_{i-1}-1}\right]$ satisfies an identity of degree $<n_{i-1}$. This implies that $l \leq n-2$ and finishes the proof of the lemma.

Let $E$ be the associative commutative $F$-algebra presented by the countable set of generators $e_{i}, i \geq 1$, and relations $e_{i}^{2}=0, i \geq 1$. Ordered products $e_{\pi}=e_{i_{1}} \cdots e_{i_{r}}, \pi=\left\{i_{1}<i_{2}<\cdots<i_{r}\right\}$ form a basis of the algebra $E$. Notice that we don't consider empty products and therefore the algebra $E$ does not have 1 . 
We will start with a short overview of the rest of the proof of Theorm 1.1. The crucial role is played by the "linearized" Lie algebra $\widetilde{L}=L \otimes_{F} E$. In section 3 , we define divided polynomials: a generalization of usual Lie polynomials that make sense in the context of the Lie algebra $\widetilde{L}$. A divided polynomial is regular if it is not identically zero on any ideal $\widetilde{L^{m}}=L^{m} \otimes_{F} E, m \geq 1$. We use Lemma 3.4 to establish existence of a regular divided polynomial whose every value is divided ad-nilpotent of degree $k \geq 3$. Then we use Kostrikin-type arguments ([Kos1], Kos2], [Zel8]) to reduce $k$ to 3.

In section 4, we show how such regular divided polynomials give rise to a family of quadratic Jordan algebras. This result is new only for $p=2$ or 3 . For $p \geq 5$, it follows from [FLGGL. Using structure theory of quadratic Jordan algebras [MZ, we establish existence of a regular Jordan polynomial, every value of which is an absolute zero divisor. These references are an essential (hidden) part of the proof. This Jordan polynomial gives rise to a regular divided polynomial whose every value is divided ad-nilpotent of degree 2, i.e., is a sandwich (see Kos2, $[\mathrm{KZ}]$ ).

In sections 5 and 6, we further push the envelope and construct a regular divided polynomial whose every value generates a nilpotent ideal in an associative enveloping algebra to reduce the problem to the case when the associative enveloping algebra $\widetilde{A}$ satisfies a polynomial identity and finish the proof using structure theory of PI-algebras.

For an arbitrary (not necessarily associative) $F$-algebra $A$ and its Lie algebra of derivations $D=\operatorname{Der}(A)$ denote $\widetilde{A}=A \otimes_{F} E, \widetilde{D}=D \otimes_{F} E$. Clearly, $\widetilde{D} \subseteq \operatorname{Der}(\widetilde{A})$. Let $i \in N$ and let $\widetilde{D_{i}}=\sum_{\pi} D \otimes e_{\pi}$, where the sum is taken over all ordered subsets of $\pi$ that contain $i$. Clearly, $\widetilde{D_{i}} \triangleleft \widetilde{D}$, $\widetilde{D}_{i}^{2}=(0),\left(A D_{i}\right)\left(A D_{i}\right)=(0)$, and $\widetilde{D}=\sum_{i} \widetilde{D_{i}}$.

Let $\Omega$ be a finite family of elements of $\widetilde{D}$ such that

(U1) every element $d \in \Omega$ lies in some ideal $\widetilde{D_{i}}$, (U2) $\left[d_{1}, d_{2}\right]=0$ for arbitrary elements $d_{1}, d_{2} \in \Omega$. 
Consider the following linear operator on $\widetilde{A}$ :

$$
U_{k}(\Omega)=\sum d_{1} \cdots d_{k}
$$

where the summation runs over all $k$-element subsets of $\Omega$.

We further define $U_{0}(\Omega)=$ Id. Clearly, $U_{1}(\Omega)=\sum_{d \in \Omega} d$.

We have $k ! U_{k}(\Omega)=\left(\sum_{d \in \Omega} d\right)^{k}$ and if the characteristic of the field exceeds $k$ then

$$
U_{k}(\Omega)=\frac{1}{k !}\left(\sum_{d \in \Omega} d\right)^{k} .
$$

Hence the operators $U_{k}$ play the role of divided powers.

The following properties of the operators $U_{k}(\Omega)$ are straightforward (see also [Zel5, Zel7]).

\section{Lemma 3.7.}

(1) $(a b) U_{m}(\Omega)=\sum_{i=0}^{m}\left(a U_{i}(\Omega)\right)\left(b U_{m-i}(\Omega)\right)$ for arbitrary elements $a, b \in \widetilde{A}, m \geq 0 ;$

(2) The operator $\widetilde{A} \rightarrow \widetilde{A}, a \rightarrow \sum_{i=0}^{\infty} a U_{i}(\Omega)$ is an automorphism of the algebra $\widetilde{A}$. We remark that the sum $\sum_{i=0}^{\infty} a U_{i}(\Omega)$ is finite;

(3) For an element $a \in \widetilde{A}$ let $R(a)$ denote the operator of right multiplication by a. Then

$$
R\left(a U_{m}(\Omega)\right)=\sum_{i=0}^{m}(-1)^{i} U_{i}(\Omega) R(a) U_{m-i}(\Omega) ;
$$

(4) $U_{i}(\Omega) U_{j}(\Omega)=\left(\begin{array}{c}i+j \\ i\end{array}\right) U_{i+j}(\Omega)$.

Remark. This lemma will be primarily applied to Lie algebras where the operator $R(a)$ of right multiplication by an element $a$ is the adjoint operator $\operatorname{ad}(a)$.

An arbitrary element $a \in \widetilde{A}$ can be uniquely represented as $a=\sum a_{\pi}$ where $a_{\pi} \in A \otimes e_{\pi}$. We call it the standard decomposition of $a$. 
Let $X$ be a countable set and let $I$ be an ideal of a Lie algebra $L$. Consider the set $\operatorname{Map}(X, \widetilde{I})$ of mappings $X \rightarrow \widetilde{I}=I \otimes_{F} E$. Consider also $M(I)=\operatorname{Map}(\operatorname{Map}(X, \widetilde{I}), \widetilde{I})$. In other words, if $f \in M(I)$ and we assign values from $\widetilde{I}$ to variables from $X$ then $f$ takes values in $\widetilde{I}$.

Let $\operatorname{Lie}\langle X\rangle$ be the free Lie algebra on the set of free generators $X$. Consider the free product $L * \operatorname{Lie}\langle X\rangle$. Let $(X)$ be the ideal of the algebra $L * \operatorname{Lie}\langle X\rangle$ generated by $X$. An arbitrary element from $(X)$ gives rise to an element from $M(I)$.

We will define a subset $U(I) \subseteq M(I)$ that we will call the set of divided polynomials defined on $I$ :

(DP1) All elements from $(X)$ lie in $U(I)$;

(DP2) suppose that a divided polynomial $w$ does not depend on any variables except $x_{1}, \cdots, x_{r}$. We represent this fact as $w=$ $w\left(x_{1}, \cdots, x_{r}\right)$. If $v_{1}, \cdots, v_{r} \in U(I)$, then $w\left(v_{1}, \cdots, v_{r}\right) \in U(I)$ as well.

If $w=w\left(x_{1}, \cdots, x_{r}\right)$ and $v_{i}=v_{i}\left(y_{1}, \cdots, y_{m}\right), 1 \leq i \leq r$, are homogeneous divided polynomials of degrees $\operatorname{deg}_{x_{i}}(w), \operatorname{deg}_{y_{k}}\left(v_{i}\right)$ in each variable, then $w\left(v_{1}, \cdots, v_{r}\right)$ is a homogeneous divided polynomial of degrees $\sum_{i=1}^{r} \operatorname{deg}_{x_{i}}(w) \cdot \operatorname{deg}_{y_{k}}\left(v_{i}\right)$ in $y_{k}, 1 \leq k \leq m$;

(DP3) let $w=w\left(x_{1}, \cdots, x_{r}\right) \in U(I)$. Suppose that

i) for arbitrary elements $a, b, a_{2}, \cdots, a_{r} \in \widetilde{I}$, we have

$$
\left[w\left(a, a_{2}, \cdots, a_{r}\right), w\left(b, a_{2}, \cdots, a_{r}\right)\right]=0 ;
$$

ii) $w$ is linear in $x_{1}$, which means that $w\left(\alpha a+\beta b, a_{2}, \cdots, a_{r}\right)=$ $\alpha w\left(a, a_{2}, \cdots, a_{r}\right)+\beta w\left(b, a_{2}, \cdots, a_{r}\right)$ for arbitrary $\alpha, \beta \in E$; $a, b, a_{2}, \cdots, a_{r} \in \widetilde{I}$ and

$$
w\left(I \otimes e_{\pi}, a_{2}, \cdots, a_{r}\right) \subseteq I \otimes e_{\pi}+I \otimes e_{\pi} E .
$$

Then for an arbitary $k \geq 0$ the function $w^{\prime}=x_{0} \operatorname{ad}_{x_{1}}^{[k]}(w)$, where $w^{\prime}$ is defined as

$$
\begin{aligned}
& w^{\prime}\left(a_{0}, a_{1}, \cdots, a_{r}\right)=a_{0} U_{k}(\Omega), \\
& \Omega=\left\{\operatorname{ad}\left(w\left(a_{1 \pi}, a_{2}, \cdots, a_{r}\right)\right\}_{\pi},\right.
\end{aligned}
$$


where $a_{1}=\sum_{\pi} a_{1 \pi}$ in the standard decomposition of the element $a_{1}$, is a divided polynomial defined on $I$.

If $w$ is a homogeneous divided polynomial of degrees $\operatorname{deg}_{x_{i}}(w)$ in $x_{1}, \cdots, x_{r}$, then $w^{\prime}$ is a homogeneous divided polynomial of degrees $1, \kappa \cdot \operatorname{deg}_{x_{i}}(w), 1 \leq i \leq r$, in $x_{0}, x_{1}, x_{2}, \cdots, x_{r}$.

An element of $M(I)$ lies in $U(I)$ if and only if starting with elements from $(X)$ and using rules DP2-DP3, it can be shown to be a divided polynomial.

A divided polynomial from $U(I)$ is a homogeneous divded polynomial if and only if starting with homogeneous elements from $(X)$ and applying rules DP2-DP 3 to homogeneous polynomials, it can be shown to be a homogeneous divided polynomial.

Let's recall the definition of a polynomial map of vector spaces.

Let $V, W$ be vector spaces over an infinite field $F$ and let $f: \underbrace{V \times \cdots \times V}_{m} \rightarrow W,\left(v_{1}, \cdots, v_{m}\right) \rightarrow f\left(v_{1}, \cdots, v_{m}\right) \in W$. If $f$ is multilinear then it is said to be a polynomial of degrees $(1,1, \cdots, 1)$ in $v_{1}, \cdots, v_{m}$. Let $d_{i} \geq 1, i=1, \cdots, m$. We say that $f$ is a homogeneous polynomial map of degrees $\left(d_{1}, \cdots, d_{m}\right)$ in $v_{1}, \cdots, v_{m}$ if

(1) for $d_{i}=1 f$ is linear in $v_{i}$;

(2) for $d_{i} \geq 2$ we have

$$
\begin{gathered}
f\left(v_{1}, \cdots, v_{i-1}, v_{i}^{\prime}+v_{i}^{\prime \prime}, v_{i+1}, \cdots, v_{m}\right)-f\left(v_{1}, \cdots, v_{i-1}, v_{i}^{\prime}, v_{i+1}, \cdots, v_{m}\right) \\
\quad-f\left(v_{1}, \cdots, v_{i-1}, v_{i}^{\prime \prime}, v_{i+1}, \cdots, v_{m}\right) \\
=\sum_{k=1}^{d_{i}-1} f_{k}\left(v_{1}, \cdots, v_{i-1}, v_{i}^{\prime}, v_{i}^{\prime \prime}, v_{i+1}, \cdots, v_{m}\right),
\end{gathered}
$$

where $f_{k}\left(v_{1}, \cdots, v_{i-1}, v_{i}^{\prime}, v_{i}^{\prime \prime}, v_{i+1}, \cdots, v_{m}\right)$ is a homogeneous polynomial map of degrees $d_{1}, \cdots, d_{i-1}, k, d_{i}-k, d_{i+1}, \cdots, d_{m}$ in $v_{1}, \cdots, v_{i-1}, v_{i}^{\prime}, v_{i}^{\prime \prime}, v_{i+1}, \cdots, v_{m}$.

Now recall the definition of a full linearization of a homogeneous polynomial map $f$ of degrees $d_{1} \geq 1, \cdots, d_{m} \geq 1$ in $v_{1}, \cdots, v_{m}$. For every $1 \leq i \leq m$ choose $d_{i}$ elements $v_{i 1}, \cdots, v_{i d_{i}} \in V$. Let $\pi \subseteq$ 
$\left\{v_{i 1}, \cdots, v_{i d_{i}}\right\}$ be a nonempty subset. Denote

$f_{\pi}=f\left(v_{1}, \cdots, v_{i-1}, \sum_{v \in \pi} v, v_{i+1}, \cdots, v_{m}\right)$. The mapping

$$
\Delta_{i}(f)=\sum_{\varnothing \neq \pi \subseteq\left\{v_{i 1}, \cdots, v_{i d_{i}}\right\}}(-1)^{\left(d_{i}-|\pi|\right)} f_{\pi}
$$

is called the linearization of $f$ with respect to $v_{i}$. The mapping $\Delta_{i}(f)\left(v_{1}, \cdots, v_{i-1}, v_{i 1}, \cdots, v_{i d_{i}}, v_{i+1}, \cdots, v_{m}\right)$ is multilinear in $v_{i 1}, \cdots, v_{i d_{i}}$.

Consecutively applying linearizations with respect to all variables, we get the full linearization $\tilde{f}: \underbrace{V \times \cdots \times V}_{\sum_{i=1}^{m} d_{i}} \rightarrow W$. Clearly $\tilde{f}$ is a multilinear map.

Lemma 3.8. For an arbitrary homogeneous divided polynomial $w \in$ $U(I)$

1) the full linearization $\widetilde{w}$ of $w$ lies in $L *$ Lie $\langle X\rangle$;

2) the span of all values of $w$ on $\widetilde{I}$ is equal to the span of all values of $\widetilde{w}$ on $\widetilde{I}$.

Proof. 1) We will use induction on the number of steps DP2-DP3 needed to construct the divided polynomial $w$.

If $v_{1}, \cdots, v_{r}, w$ are homogeneous divided polynomials, then the full linearization of $w\left(v_{1}, \cdots, v_{r}\right)$ is a linear combination of values $\widetilde{w}\left(\widetilde{v_{1}}, \cdots, \widetilde{v_{r}}\right)$ in appropriate variables.

Let $w=x_{0} \operatorname{ad}_{x_{1}}^{[k]}(v)$, where $v=v\left(x_{1}, \cdots, x_{r}\right)$ in a homogeneous divided polynomial satisfying the conditions DP3 (i), (ii). Since we can linearize variables in an arbitrary order, let's start with the variable $x_{1}$. Then

$$
\begin{gathered}
\Delta_{x_{1}}(w)\left(x_{0}, y_{1}, \cdots, y_{k}, x_{2}, \cdots, x_{r}\right) \\
=x_{0} \operatorname{ad}\left(v\left(y_{1}, x_{2}, \cdots, x_{r}\right)\right) \cdots \operatorname{ad}\left(v\left(y_{k}, x_{2}, \cdots, x_{r}\right)\right) .
\end{gathered}
$$

This completes the proof of assertion 1).

We will prove part 2) of the Lemma in a slightly more general context of polynomial maps of spaces. Consider again vector spaces $V, W$ and a 
homogeneous polynomial map $f: \underbrace{V \times \cdots \times V}_{m} \rightarrow W,\left(x_{1}, \cdots, x_{m}\right) \rightarrow$ $f\left(x_{1}, \cdots, x_{m}\right) \in W, x_{i} \in V$. Let $f$ have degrees $d_{1} \geq 1, \cdots, d_{m} \geq 1$ with respect to $x_{1}, \cdots, x_{m}$. Choose $x_{i}^{\prime}, x_{i}^{\prime \prime} \in V, 1 \leq i \leq m$. Consider

$$
\begin{gathered}
f\left(x_{1}, \cdots, x_{i-1}, x_{i}^{\prime}+x_{i}^{\prime \prime}, x_{i+1}, \cdots, x_{m}\right)-f\left(x_{1}, \cdots, x_{i-1}, x_{i}^{\prime}, x_{i+1}, \cdots, x_{m}\right) \\
\quad-f\left(x_{1}, \cdots, x_{i-1}, x_{i}^{\prime \prime}, x_{i+1}, \cdots, x_{m}\right) \\
=\sum_{j=1}^{d_{1}-1} f_{j}\left(x_{1}, \cdots, x_{i-1}, x_{i}^{\prime}, x_{i}^{\prime \prime}, x_{i+1}, \cdots, x_{m}\right),
\end{gathered}
$$

where the summand $f_{j}$ has degree $j$ in $x_{i}^{\prime}$ and degree $d_{i}-j$ in $x_{i}^{\prime \prime}$. The homogeneous polynomial mappings $f_{j}$ are called partial linearizations of $f$.

Consider the finite system $\mathcal{F}$ of homogeneous polynomial maps from $V$ to $W$ that are obtained from $f$ by repeated partial linearizations.

Let $\Omega \subset V$ be a family of elements with the following property:

$(*) \quad$ if $g\left(x_{1}, \cdots, x_{r}\right) \in \mathcal{F}$ and $g$ has degree $\geq 2$ in $x_{i}$, then for an arbitrary element $v \in \Omega$ we have

$$
g(\underbrace{V, \cdots, V}_{i-1}, v, \underbrace{V, \cdots, V}_{r-i})=(0) .
$$

We claim that for an arbitrary element $g\left(x_{1}, \cdots, x_{r}\right) \in \mathcal{F}$,

$$
g(\operatorname{span} \Omega, \cdots, \operatorname{span} \Omega) \subseteq \operatorname{span} \tilde{f}(\Omega, \cdots, \Omega) .
$$

Applying this inclusion to $f=w, \Omega=\bigcup_{i \geq 1}\left(I \otimes e_{i}+I \otimes e_{i} E\right)$, we will prove part 2) of Lemma 3.8 .

If $g$ is multilinear, then $g=\tilde{f}$. In any case, without loss of generality, we assume that the claim is true for all partial linearizations of $g$. But modulo partial linearizations the mapping $g$ is multilinear. More precisely,

$$
\begin{gathered}
g(\operatorname{span} \Omega, \cdots, \operatorname{span} \Omega) \subseteq \\
\operatorname{span}\left(g\left(v_{1}, \cdots, v_{r}\right), v_{i} \in \Omega\right)+\sum g^{\prime}(\operatorname{span} \Omega, \cdots, \operatorname{span} \Omega),
\end{gathered}
$$

where $g^{\prime}$ are partial linearizations of $g$. Since $g$ has degree $\geq 2$ with respect to at least one variable, we conclude that $g\left(v_{1}, \cdots, v_{r}\right)=0$. 
In particular,

$$
\operatorname{span} f(\operatorname{span} \Omega, \cdots, \operatorname{span} \Omega)=\operatorname{span}\left(\tilde{f}\left(v_{1}, v_{2}, \cdots\right), v_{i} \in \Omega\right) .
$$

If $f$ is a homogeneous divided polynomial and $\Omega=\left\{a \otimes e_{i}, a \in L, i \geq 1\right\}$, then condition (因) is clearly satisfied, which completes the proof of assertion 2).

The following lemma is a linearization version of the celebrated Kostrikin Lemma ([Kos1; Kos2, Lemma 2.1.1]).

Lemma 3.9. Let $L$ be a Lie algebra. Let $\Omega \subset \operatorname{Der}(L) \otimes E$ be a finite

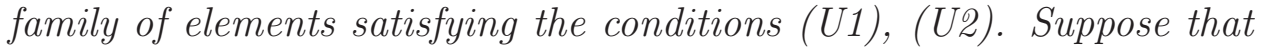
$m \geq 1$ and for an arbitary $k \geq m$, we have $U_{k}(\Omega)=0$.

1) Let $m \geq 2$. Then for arbitary elements $a, b \in L$, we have

$$
\left[a U_{m-1}(\Omega), b U_{m-1}(\Omega)\right]=0 .
$$

2) Now suppose that $m \geq 4$. Let $a \in \widetilde{L}, a=\sum_{\pi} a_{\pi}$ be a standard decomposition, and $\Omega^{\prime}=\left\{a_{\pi} U_{m-1}(\Omega)\right\}_{\pi}$. Then $U_{k}\left(\Omega^{\prime}\right)=0$ for $k \geq$ $m-1$.

Proof. 1) We have $2 m-2 \geq m$. Adjoint operators are right multiplications in Lie algebras. Hence Lemma 3.7 (3) is applicable. By Lemma $3.7(3)$,

$$
0=\operatorname{ad}\left(b U_{2 m-2}(\Omega)\right)=\sum_{i+j=2 m-2} \pm U_{i}(\Omega) \operatorname{ad}(b) U_{j}(\Omega) .
$$

It implies

$$
U_{m-1}(\Omega) \operatorname{ad}(b) U_{m-1}(\Omega)=\sum_{\substack{i \geq m \\ \text { or } j \geq m}} \pm U_{i}(\Omega) \operatorname{ad}(b) U_{j}(\Omega)=0 .
$$

By Lemma 3.7 (3), (4), we have

$$
\begin{aligned}
{\left[a U_{m-1}(\Omega), b U_{m-1}(\Omega)\right] } & =a U_{m-1}(\Omega) \operatorname{ad}\left(b U_{m-1}(\Omega)\right) \\
& =a U_{m-1}(\Omega) \operatorname{ad}(b) U_{m-1}(\Omega) \\
& =0
\end{aligned}
$$

which completes the proof of the assertion 1).

2) We will show that

$$
\operatorname{ad}\left(a_{1} U_{m-1}(\Omega)\right) \cdots \operatorname{ad}\left(a_{k} U_{m-1}(\Omega)\right)=0
$$


for arbitrary elements $a_{1}, \cdots, a_{k} \in L, k \geq m-1$. Without loss of generality we will assume $k=m-1$.

By Lemma 3.7 (3) and (4) the left hand side is a linear combination of generators

$$
U_{i_{0}}(\Omega) \operatorname{ad}\left(a_{1}\right) U_{i_{1}}(\Omega) \cdots U_{i_{m-2}}(\Omega) \operatorname{ad}\left(a_{m-1}\right) U_{i_{m-1}}(\Omega),
$$

where $0 \leq i_{0}, i_{1}, \cdots, i_{m-1} \leq m-1$ and $i_{0}+i_{1}+\cdots+i_{m-1}=(m-1)^{2}$.

Suppose that $U_{i_{0}}(\Omega) \operatorname{ad}\left(a_{1}\right) \cdots \operatorname{ad}\left(a_{m-1}\right) U_{i_{m-1}}(\Omega) \neq 0$ and the $m$ tuple $\left(i_{0}, i_{1}, \cdots, i_{m-1}\right)$ is lexicographically maximal with this property.

We claim that none of the indices $i_{0}, i_{1}, \cdots, i_{m-1}$ are equal to 0 . Indeed, if one of the indices is equal to 0 , then all other indices have to be equal to $m-1$. Since $m \geq 4$ it follows that there exists $t$, $0 \leq t \leq m-1$, such that $i_{t}=i_{t+1}=m-1$. Now from 1) it follows that $U_{i_{t}}(\Omega) \operatorname{ad}\left(a_{t+1}\right) U_{i_{t+1}}(\Omega)=U_{m-1}(\Omega) \operatorname{ad}\left(a_{t+1}\right) U_{m-1}(\Omega)=0$, a contradiction.

Since $(m-2) m<(m-1)^{2}$ it follows that at least one index $i_{t}$, $0 \leq t \leq m-1$, is equal to $m-1$. All of the indices $i_{1}, \cdots, i_{m-1}$ are smaller than $m-1$. Indeed, suppose that $i_{t}=m-1,1 \leq t \leq m-1$. We have $i_{t-1} \geq 1$ by the above. Now Lemma 3.7 (3) implies

$$
0=\operatorname{ad}\left(a_{t} U_{i_{t-1}+i_{t}}(\Omega)\right)=\sum_{i+j=i_{t-1}+i_{t}} \pm U_{i}(\Omega) \operatorname{ad}\left(a_{t}\right) U_{j}(\Omega)
$$

and therefore

$$
U_{i_{t-1}}(\Omega) \operatorname{ad}\left(a_{t}\right) U_{i_{t}}(\Omega)=\sum_{\substack{i>i_{t} \\ j<m-1}} \pm U_{i}(\Omega) \operatorname{ad}\left(a_{t}\right) U_{j}(\Omega),
$$

which contradicts lexicographical maximality of $\left(i_{0}, \cdots, i_{m-1}\right)$.

We have proved that $i_{0}=m-1, i_{1}=i_{2}=\cdots=i_{m-1}=m-2$. Now our aim will be to show that

$$
U_{m-1}(\Omega) \operatorname{ad}\left(a_{1}\right) U_{m-2}(\Omega)=0 .
$$

Since $(m-1)+(m-3) \geq m$, Lemma 3.7 (3) implies that

$$
\begin{gathered}
U_{m-1}(\Omega) \operatorname{ad}\left(a_{1}\right) U_{m-3}(\Omega)-U_{m-2}(\Omega) \operatorname{ad}\left(a_{1}\right) U_{m-2}(\Omega) \\
+U_{m-3}(\Omega) \operatorname{ad}\left(a_{1}\right) U_{m-1}(\Omega)=0 .
\end{gathered}
$$


Multiplying the left hand side by $U_{1}(\Omega)$ on the right and taking into account Lemma 3.7 (4), we get

$(m-2) U_{m-1}(\Omega) \operatorname{ad}\left(a_{1}\right) U_{m-2}(\Omega)-(m-1) U_{m-2}(\Omega) \operatorname{ad}\left(a_{1}\right) U_{m-1}(\Omega)=0$.

On the other hand Lemma 3.7 (3) implies that

$$
U_{m-1}(\Omega) \operatorname{ad}\left(a_{1}\right) U_{m-2}(\Omega)-U_{m-2}(\Omega) \operatorname{ad}\left(a_{1}\right) U_{m-1}(\Omega)=0 .
$$

The system of equations implies

$$
U_{m-1}(\Omega) \operatorname{ad}\left(a_{1}\right) U_{m-2}(\Omega)=0,
$$

which completes the proof of the lemma.

Definition 3.10. We say that a divided polynomial $w\left(x_{1}, \cdots, x_{r}\right)$ defined on $L^{s}, s \geq 1$, is regular if for an arbitrary $i \geq s$ we have $w\left(\widetilde{L}^{i}, \cdots, \widetilde{L}^{i}\right) \neq(0)$.

By Lemma 3.8 (2), a homogeneous divided polynomial $w$ is regular if and only if its full linearization is regular.

Lemma 3.11. There exist integers $m \geq 1, N \geq 1$ and a regular homogeneous divided polynomial $w\left(x_{1}, \cdots, x_{r}\right)$ defined on $L^{m}$ such that $w$ satisfies the conditions in (DP3) and $x_{0} \operatorname{ad}_{x_{1}}^{[t]}(w)=0$ holds identically on $\widetilde{L}^{m}$ for all $t \geq N$.

Proof. Consider the elements $c_{1}, \cdots, c_{r} \in S$ and integers $m \geq 1, N \geq 1$ of Lemma 3.4. By property 2), for an $i \geq m$ the subspace $L_{i}=$ $\left[L^{i}, c_{1}, \cdots, c_{r}\right]$ is a subalgebra of $L$. By 3$)$ this subalgebra is nilpotent, say, of degree $d(i), d(m) \geq d(m+1) \geq \cdots$. This sequence stabilizes at some step, $d=d(k)=d(k+1)=\cdots$. Thus $L_{k}^{d}=(0)$ and $L_{i}^{d-1} \neq(0)$ for any $i \geq m$. Let

$$
\begin{aligned}
w\left(x_{1}, \cdots, x_{d-1}\right)=\left[\left[x_{1}, c_{1}, \cdots, c_{r}\right],\left[x_{2}, c_{1}, \cdots, c_{r}\right], \cdots\right. \\
\left.\cdots,\left[x_{d-1}, c_{1}, \cdots, c_{r}\right]\right] \in L * \operatorname{Lie}\langle X\rangle .
\end{aligned}
$$

The divided polynomial $w$ is regular and linear in $x_{1}$. For arbitrary elements $a_{2}, \cdots, a_{d-1} \in L^{k}$ we have

$$
\left[w\left(L^{k}, a_{2}, \cdots, a_{d-1}\right), w\left(L^{k}, a_{2}, \cdots, a_{d-1}\right)\right]=(0)
$$

because the left hand side lies in $L_{k}^{d}$. Hence the divided polynomial $w$ satisfies the condition (DP3). Therefore the divided polynomial $x_{0} \operatorname{ad}_{x_{1}}^{[t]}(w)$ is defined on $L^{k}$ for any $t \geq 1$. For $t \geq N$ the polynomial $x_{0} \operatorname{ad}_{x_{1}}^{[t]}(w)$ is identically zero on $\widetilde{L}^{k}$ by Lemma 3.4 (3). This finishes the proof of the lemma. 
Let $q \geq 1$ be a minimal integer with the following property:

there exists an $m \geq 1$ and a regular homogeneous divided polynomial $w=w\left(x_{1}, \cdots, x_{r}\right)$ defined on $L^{m}$, linear in $x_{1}$, such that

i) for arbitrary elements $a, b, a_{2}, \cdots, a_{r} \in \widetilde{L}^{m}$ we have

$$
\left[w\left(a, a_{2}, \cdots, a_{r}\right), w\left(b, a_{2}, \cdots, a_{r}\right)\right]=0 ;
$$

ii) $\widetilde{L}^{m} \operatorname{ad}_{x 1}^{[t]}(w)=(0)$ holds identically on $\widetilde{L}^{m}$ for all $t \geq q$. Clearly, $q \leq N$.

Lemma 3.12. $q \leq 3$.

Proof. Suppose that $q \geq 4$. Consider the divided polynomial $v\left(x_{0}, x_{1}, \cdots, x_{r}\right)=x_{0} \operatorname{ad}_{x_{1}}^{[q-1]}(w)$ defined on $L^{m}$. In view of the minimality of $q$, the divided polynomial $v$ is regular.

By Lemma 3.9 (1) for arbitrary elements $a, b \in \widetilde{L}^{m} ; a_{1}, \cdots, a_{r} \in \widetilde{L}^{m}$ we have

$$
\left[v\left(a, a_{1}, \cdots, a_{r}\right), v\left(b, a_{1}, \cdots, a_{r}\right)\right]=0 .
$$

We proved that the divided polynomial $y \operatorname{ad}_{x_{0}}^{[q-1]}\left(v\left(x_{0}, \cdots, x_{r}\right)\right)$ is defined on $\widetilde{L}^{m}$. If $q \geq 4$, then by Lemma 3.9 (2), this divided polynomial is identically zero, which contradicts the minimality of $q$ and finishes the proof of the lemma.

Lemma 3.13. Let $L$ be a Lie algebra. Let $\Omega \subset \widetilde{L}$ be a finite family of

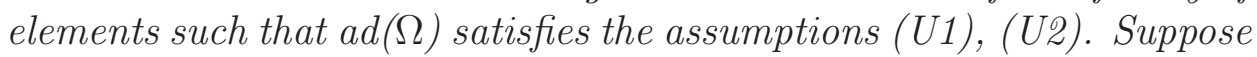
that $U_{2}(a d(\Omega))=0$. Then $a=\sum_{b \in \Omega} b$ is a sandwich of the Lie algebra $\widetilde{L}$.

Proof. We have $\operatorname{ad}(a)^{2}=U_{1}(\operatorname{ad}(\Omega)) U_{1}(\operatorname{ad}(\Omega))=2 U_{2}(\operatorname{ad}(\Omega))=0$. By Lemma 3.7 (3) for an arbitrary element $c \in \widetilde{L}$ we have

$$
\begin{aligned}
\operatorname{ad}\left(c U_{2}(\operatorname{ad}(\Omega))\right)=\operatorname{ad}(c) U_{2}(\operatorname{ad}(\Omega))-U_{1}(\operatorname{ad}(\Omega)) \operatorname{ad}(c) & U_{1}(\operatorname{ad}(\Omega)) \\
& +U_{2}(\operatorname{ad}(\Omega)) \operatorname{ad}(c),
\end{aligned}
$$

which implies $\operatorname{ad}(b) \operatorname{ad}(c) \operatorname{ad}(b)=0$ and completes the proof of the lemma.

In what follows, we will use the subsequent lemma. 
Lemma 3.14. Let $L$ be a Lie algebra. Let $\Omega=\left\{a_{1}, \cdots, a_{n}\right\} \subset L$ be a finite family of elements. Let $\Omega=\Omega_{1} \dot{\cup} \cdots \dot{\cup} \Omega_{s}=\Omega_{1}^{\prime} \dot{\cup} \cdots \dot{U} \Omega_{t}^{\prime}$ be two disjoint decompositions. Denote $b_{k}=\sum_{a_{i} \in \Omega_{k}} a_{i}, c_{\ell}=\sum_{a_{j} \in \Omega_{\ell}^{\prime}} a_{j}$. Denote also $\operatorname{ad}[\Omega, \Omega]=\operatorname{Span}\left(\operatorname{ad}\left[a_{i}, a_{j}\right], 1 \leq i, j \leq n\right)$.

Suppose that if $a_{i}, a_{j}$ lie in the same $\Omega_{k}$ or in the same $\Omega_{\ell}^{\prime}$, then $\operatorname{ad}\left(a_{i}\right) \operatorname{ad}\left(a_{j}\right)=0$. Then $\sum \operatorname{ad}\left(b_{k_{1}}\right) \operatorname{ad}\left(b_{k_{2}}\right)=\sum \operatorname{ad}\left(c_{\ell_{1}}\right) \operatorname{ad}\left(c_{\ell_{2}}\right) \bmod$ $\operatorname{ad}[\Omega, \Omega]$, where both sums run over all 2-element subsets $\left\{k_{1}, k_{2}\right\} \subseteq$ $\{1, \cdots, s\}$ and $\left\{\ell_{1}, \ell_{2}\right\} \subseteq\{1,2, \cdots, t\}$ respectively.

Proof. It is easy to see that

$$
\begin{aligned}
\sum \operatorname{ad}\left(b_{k_{1}}\right) \operatorname{ad}\left(b_{k_{2}}\right) & =\sum \operatorname{ad}\left(c_{\ell_{1}}\right) \operatorname{ad}\left(c_{\ell_{2}}\right) \\
& =\sum_{1 \leq i<j \leq n} \operatorname{ad}\left(a_{i}\right) \operatorname{ad}\left(a_{j}\right) \quad \bmod \operatorname{ad}[\Omega, \Omega] .
\end{aligned}
$$

\section{Jordan ALGEBRAS}

Let $J$ be a vector space over a field $F$ (of arbitrary characteristic) with two quadratic mappings $J \rightarrow J, x \rightarrow x^{2}$, and $Q: J \rightarrow \operatorname{End}_{F}(J)$. For elements $x, y, z \in J$ denote

$$
x \circ y=(x+y)^{2}-x^{2}-y^{2},\{x, y, z\}=y(Q(x+z)-Q(x)-Q(z)) .
$$

Following K. McCrimmon ([McC1]) we say that $\left(J, x \rightarrow x^{2}, Q\right)$ is a quadratic Jordan algebra if it satisfies the identities

(M1) $\{x, x, y\}=x^{2} \circ y$;

(M2) $(y Q(x)) \circ x=(y \circ x) Q(x)$;

(M3) $x^{2} Q(x)=\left(x^{2}\right)^{2}$;

(M4) $x^{2} Q(y) Q(x)=(y Q(x))^{2}$;

(M5) $Q\left(x^{2}\right)=Q(x)^{2}$;

(M6) $Q(y Q(x))=Q(x) Q(y) Q(x)$

and all their partial linearizations.

We reiterate the assumption made at the beginning of section 3: all algebras are considered over an infinite field $F$ of characteristic $p>0$. 
Let $w^{\prime}=w^{\prime}\left(x_{1}, \cdots, x_{r-1}\right)$ be a regular homogeneous divided polynomial defined on $L^{m}$ such that $w^{\prime}$ is linear in $x_{1}$ and satisfies all the assumptions of (DP[3). Moreover, assume that $\widetilde{L^{m}} \operatorname{ad}_{x_{1}}^{[k]}\left(w^{\prime}\right)=0$ holds identically for $k \geq 3$. If there exists $s \geq m$ such that $x_{r} \operatorname{ad}_{x_{1}}^{[2]}\left(w^{\prime}\right)$ holds identically on $\widetilde{L^{s}}$ then our goal of constructing a sandwich valued regular homogeneous divided polynomial has been achieved. We assume therefore that the divided polynomial $w\left(x_{1}, \cdots, x_{r}\right)=x_{r} \operatorname{ad}_{x_{1}}^{[2]}\left(w^{\prime}\right)$ is regular.

The divided polynomial $w$ satisfies both assumptions of (DP3): it is clearly linear in $x_{r}$ and for arbitrary elements $a, b, a_{1}, \cdots, a_{r-1} \in \widetilde{L^{m}}$, we have

$$
\left[a \operatorname{ad}_{x_{1}}^{[2]} w^{\prime}\left(a_{1}, \cdots, a_{r-1}\right), b \operatorname{ad}_{x_{1}}^{[2]} w^{\prime}\left(a_{1}, \cdots, a_{r-1}\right)\right]=0
$$

by Lemma 3.9 (1).

Choose $a_{1}, \cdots, a_{r} \in \widetilde{L^{m}}$ and denote $a^{\prime}=w^{\prime}\left(a_{1}, \cdots, a_{r-1}\right)$, $a=w\left(a_{1}, \cdots, a_{r}\right)$. Denote $\operatorname{ad}^{[k]}\left(a^{\prime}\right)=\left(\operatorname{ad}_{x_{1}}^{[k]} w^{\prime}\right)\left(a_{1}, \cdots, a_{r-1}\right)$. If $a_{r}=\sum_{\pi} a_{r \pi}$ is the standard decomposition, then we denote $\operatorname{ad}^{[k]}(a)=$ $\left(\operatorname{ad}_{x_{r}}^{[k]} w\right)\left(a_{1}, \cdots, a_{r}\right)=\sum \operatorname{ad}\left(a_{r \pi_{1}} \operatorname{ad}^{[2]}\left(a^{\prime}\right)\right) \cdots \operatorname{ad}\left(a_{r \pi_{k}} \operatorname{ad}^{[2]}\left(a^{\prime}\right)\right)$, where the sum runs over all $k$-element sets $\left(\pi_{1}, \cdots, \pi_{k}\right)$.

Notice that $\operatorname{ad}^{[k]}(a)=0$ for $k \geq 3$. Indeed, by Lemma 3.7 (3), the equalities $\operatorname{ad}\left(a_{r \pi_{i}} \operatorname{ad}^{[3]}\left(a^{\prime}\right)\right)=0$ and $\operatorname{ad}\left(a_{r \pi_{i}} \operatorname{ad}^{[4]}\left(a^{\prime}\right)\right)=0$ imply

$$
\operatorname{ad}^{[2]}\left(a^{\prime}\right) \operatorname{ad}\left(a_{r \pi_{i}}\right) \operatorname{ad}\left(a^{\prime}\right)=\operatorname{ad}\left(a^{\prime}\right) \operatorname{ad}\left(a_{r \pi_{i}}\right) \operatorname{ad}^{[2]}\left(a^{\prime}\right),
$$

$$
\operatorname{ad}^{[2]}\left(a^{\prime}\right) \operatorname{ad}\left(a_{r \pi_{i}}\right) \operatorname{ad}^{[2]}\left(a^{\prime}\right)=0
$$

respectively.

We have

$$
\operatorname{ad}^{[k]}(a)=\sum \pm \operatorname{ad}^{\left[i_{1}\right]}\left(a^{\prime}\right) \operatorname{ad}\left(a_{r \pi_{1}}\right) \operatorname{ad}^{\left[i_{2}\right]}\left(a^{\prime}\right) \cdots \operatorname{ad}\left(a_{r \pi_{k}}\right) \operatorname{ad}^{\left[i_{k+1}\right]}\left(a^{\prime}\right)
$$


E. ZELMANOV

where $0 \leq i_{1}, \cdots, i_{k+1} \leq 2, i_{1}+\cdots+i_{k+1}=2 k$. If at least one $i_{\mu}$, $1 \leq \mu \leq k+1$, is equal to 0 , then all other $i_{\nu}, \nu \neq \mu$, are equal to 2 . In this case, the product is equal to 0 by (**). Suppose that all $i_{\mu} \neq 0$. Then all $i_{\mu}$, except two, are equal to 2 . These two are equal to 1 . Since $k+1 \geq 4$, we have at least two degrees $i_{\mu}$ that are equal to 2 . Using

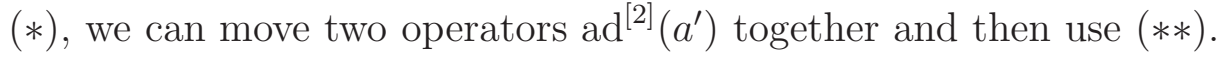

Consider the subspaces $K_{a}^{\prime}=\left\{x \in \widetilde{L}^{m} \mid x \operatorname{ad}^{[2]}(a)=0\right\}$ and $K_{a}=$ $\sum_{i}\left(L^{m} \otimes e_{i}+\widetilde{L}^{m} e_{i}\right) \cap K_{a}^{\prime}$ and the factor space $J_{a}=\widetilde{L}^{m} / K_{a}$.

Let $x=\sum_{\pi} x_{\pi}$ be the standard decomposition of an element $x \in \widetilde{L}^{m}$. Define $x^{2}=a \sum \operatorname{ad}\left(x_{\pi_{1}}\right) \operatorname{ad}\left(x_{\pi_{2}}\right)+K_{a}$, where the sum runs over all 2-element sets $\left(\pi_{1}, \pi_{2}\right)$. The order of the factors in $\operatorname{ad}\left(x_{\pi_{1}}\right) \operatorname{ad}\left(x_{\pi_{2}}\right)$ is irrelevant since $\left[a, \widetilde{L}^{m}\right] \subseteq K_{a}$. Define further

$$
y Q(x)=y \operatorname{ad}^{[2]}(a) \sum \operatorname{ad}\left(x_{\pi_{1}}\right) \operatorname{ad}\left(x_{\pi_{2}}\right)+K_{a}
$$

Again, the order of factors in $\operatorname{ad}\left(x_{\pi_{1}}\right) \operatorname{ad}\left(x_{\pi_{2}}\right)$ is irrelevant since $y \operatorname{ad}^{[2]}(a) \operatorname{ad}\left(\widetilde{L}^{m}\right) \subseteq K_{a}$.

Linearizing the above operations, we get $x \circ y=[[a, x], y]+K_{a}$ for $x, y \in J_{a}$, and $\{x, y, z\}=\left[y \operatorname{ad}^{[2]}(a), x, z\right]+K_{a}$ for $x, y, z \in J$.

\section{Lemma 4.1.}

(1) the element $u=y_{1} \operatorname{ad}^{\left[i_{1}\right]}(a) \operatorname{ad}\left(y_{2}\right) \operatorname{ad}^{\left[i_{2}\right]}(a) \cdots \operatorname{ad}\left(y_{s}\right) \operatorname{ad}^{\left[i_{s}\right]}(a)$, where $y_{1}, \cdots, y_{s} \in \widetilde{L}^{m} ; i_{1}+\cdots+i_{s} \geq s+2$, is equal to 0 ;

(2) an operator $a d^{\left[i_{1}\right]}(a) \operatorname{ad}\left(y_{1}\right) \cdots \operatorname{ad}\left(y_{s}\right) \operatorname{ad}^{\left[i_{s+1}\right]}(a)$, for $y_{1}, \cdots, y_{s} \in$ $\widetilde{L}^{m} ; i_{1}+\cdots, i_{s+1} \geq s+3$, is equal to 0 on $\widetilde{L}^{m}$

(3) consider an operator

$$
v=\operatorname{ad}^{\left[i_{1}\right]}(a) \operatorname{ad}\left(y_{1}\right) \cdots \operatorname{ad}\left(y_{s}\right) \operatorname{ad}^{\left[i_{s+1}\right]}(a),
$$

where $y_{1}, \cdots, y_{s} \in \widetilde{L}^{m}, i_{1}+\cdots+i_{s+1} \geq s+2$. Suppose that there exists $1 \leq k \leq s-2$ such that $i_{k+1}=i_{k+2}=0$, in other words $v=\sum \cdots \operatorname{ad}\left(y_{k}\right) \operatorname{ad}\left(y_{k+1}\right) \operatorname{ad}\left(y_{k+2}\right) \cdots$. Then $v$ is zero on $\widetilde{L}^{m}$.

Proof. To prove (1) we will use induction on $s$. If $s=1$ then $u=$ $y_{1} \operatorname{ad}^{\left[i_{1}\right]}(a), i_{1} \geq 3$. Hence $u=0$. 
Let $s \geq 2$. If $i_{1} \geq 3$ then again $u=0$. If $i_{1} \leq 1$ then choosing $y_{1}^{\prime}=$ $y_{1} \operatorname{ad}^{\left[i_{1}\right]}(a) \operatorname{ad}\left(y_{2}\right)$ we can use the induction assumption. Therefore we let $i_{1}=2$. If $i_{2}=0$ then choosing $y_{1}^{\prime}=i_{1} \operatorname{ad}^{[2]}(a) \operatorname{ad}\left(y_{2}\right) \operatorname{ad}\left(y_{3}\right)$ we again use the induction assumption. Let $i_{2}=1$. Then by Lemma 3.7(3) we have

$$
\operatorname{ad}^{[2]}(a) \operatorname{ad}\left(y_{2}\right) \operatorname{ad}(a)=\operatorname{ad}(a) \operatorname{ad}\left(y_{2}\right) \operatorname{ad}^{[2]}(a),
$$

the case that has already been considered. Finally, if $i_{2}=2$ then $\operatorname{ad}^{[2]}(a) \operatorname{ad}\left(y_{2}\right) \operatorname{ad}^{[2]}(a)=0$, again by Lemma 3.7(3), which finishes the proof of part (1).

To prove (2) we consider the element

$$
y_{0} \operatorname{ad}^{\left[i_{1}\right]}(a) \operatorname{ad}\left(y_{1}\right) \cdots \operatorname{ad}\left(y_{s}\right) \operatorname{ad}^{\left[i_{s+1}\right]}(a)
$$

and use part (1).

Consider now an operator $v=\operatorname{ad}^{\left[i_{1}\right]}(a) \operatorname{ad}\left(y_{1}\right) \cdots \operatorname{ad}\left(y_{s}\right) \operatorname{ad}^{\left[i_{s+1}\right]}(a)$ and suppose that

$$
v=v^{\prime} \operatorname{ad}\left(y_{k}\right) \operatorname{ad}\left(y_{k+1}\right) \operatorname{ad}\left(y_{k+2}\right) v^{\prime \prime}
$$

where

$$
\begin{gathered}
v^{\prime}=\operatorname{ad}^{\left[i_{1}\right]}(a) \operatorname{ad}\left(y_{1}\right) \cdots \operatorname{ad}\left(y_{k-1}\right) \operatorname{ad}^{\left[i_{k}\right]}(a), \\
v^{\prime \prime}=\operatorname{ad}^{\left[i_{k+3}\right]}(a) \cdots \operatorname{ad}^{\left[i_{s+1}\right]}(a) .
\end{gathered}
$$

By part (2) if $v \neq 0$ on $\widetilde{L}^{m}$ then $i_{1}+\cdots+i_{k} \leq(k-1)+2=$ $k+1, i_{k+1}+\cdots+i_{s+1} \leq(s-k-2)+2=s-k$. However, $i_{1}+\cdots+$ $i_{k}+i_{k+3}+\cdots+i_{s+1} \geq s+2$, a contradiction that finishes the proof of the lemma.

Lemma 4.2. Let $\Omega$ be a finite family of commuting elements from $\widetilde{L}$ such that every element from $\Omega$ lies in one of the ideals $L \otimes e_{\pi}+\widetilde{L} e_{\pi}$. Denote for brevity $U_{k}(\operatorname{ad}(\Omega))=\operatorname{ad}^{[k]}(\Omega)$ and suppose that $\operatorname{ad}^{[3]}(\Omega)=$ $\operatorname{ad}^{[4]}(\Omega)=0$. Then for arbitrary elements $y_{1}, y_{2} \in \widetilde{L}$ we have

$$
\operatorname{ad}\left(y_{1} \operatorname{ad}^{[2]}(\Omega)\right) \operatorname{ad}\left(y_{2} \operatorname{ad}^{[2]}(\Omega)\right)=\operatorname{ad}^{[2]}(\Omega) \operatorname{ad}\left(y_{1}\right) \operatorname{ad}\left(y_{2}\right) \operatorname{ad}^{[2]}(\Omega) .
$$

Proof. By Lemma 3.7(3) we have

$$
\begin{aligned}
& \operatorname{ad}\left(y_{i} \operatorname{ad}^{[2]}(\Omega)\right)=\operatorname{ad}\left(y_{i}\right) \operatorname{ad}^{[2]}(\Omega)- \\
& \quad \operatorname{ad}^{[1]}(\Omega) \operatorname{ad}\left(y_{i}\right) \operatorname{ad}^{[1]}(\Omega)+\operatorname{ad}^{[2]}(\Omega) \operatorname{ad}\left(y_{i}\right),
\end{aligned}
$$


$i=1,2$. By Lemma 3.7(4) we have also

$$
\begin{gathered}
\operatorname{ad}^{[1]}(\Omega) \operatorname{ad}^{[1]}(\Omega)=2 \operatorname{ad}^{[2]}(\Omega), \\
\operatorname{ad}^{[1]}(\Omega) \operatorname{ad}^{[2]}(\Omega)=\operatorname{ad}^{[2]}(\Omega) \operatorname{ad}^{[1]}(\Omega)=3 \operatorname{ad}^{[3]}(\Omega)=0 .
\end{gathered}
$$

Again by Lemma 3.7(3) we have

$$
\begin{aligned}
& \operatorname{ad}\left(y_{i} \operatorname{ad}^{[3]}(\Omega)\right)=\operatorname{ad}\left(y_{i}\right) \operatorname{ad}^{[3]}(\Omega)-\operatorname{ad}^{[1]}(\Omega) \operatorname{ad}\left(y_{i}\right) \operatorname{ad}^{[2]}(\Omega)+ \\
& \operatorname{add}^{[2]}(\Omega) \operatorname{ad}\left(y_{i}\right) \operatorname{ad}^{[1]}(\Omega)-\operatorname{ad}^{[3]}(\Omega) \operatorname{ad}\left(y_{i}\right)=0,
\end{aligned}
$$

which implies

$$
\operatorname{ad}^{[1]}(\Omega) \operatorname{ad}\left(y_{i}\right) \operatorname{ad}^{[2]}(\Omega)=\operatorname{ad}^{[2]}(\Omega) \operatorname{ad}\left(y_{i}\right) \operatorname{ad}^{[1]}(\Omega) .
$$

Similarly, $\operatorname{ad}\left(y_{i} \operatorname{ad}^{[4]}(\Omega)\right)=0$ implies ad ${ }^{[2]}(\Omega) \operatorname{ad}\left(y_{1}\right) \operatorname{ad}^{[2]}(\Omega)=0$. Hence,

$$
\begin{aligned}
& \operatorname{ad}\left(y_{1} \operatorname{ad}^{[2]}(\Omega)\right) \operatorname{ad}\left(y_{2} \operatorname{ad}^{[2]}(\Omega)\right)=\left(\operatorname{ad}\left(y_{i}\right) \operatorname{ad}^{[2]}(\Omega)-\right. \\
& \left.\operatorname{ad}^{[1]}(\Omega) \operatorname{ad}\left(y_{1}\right) \operatorname{ad}^{[1]}(\Omega)+\operatorname{ad}^{[2]}(\Omega) \operatorname{ad}\left(y_{1}\right)\right)\left(\operatorname{ad}\left(y_{2}\right) \operatorname{ad}^{[2]}(\Omega)-\right. \\
& \left.\operatorname{ad}^{[1]}(\Omega) \operatorname{ad}\left(y_{2}\right) \operatorname{ad}^{[1]}(\Omega)+\operatorname{ad}^{[2]}(\Omega) \operatorname{ad}\left(y_{2}\right)\right)= \\
& -\operatorname{ad}^{[1]}(\Omega) \operatorname{ad}\left(y_{1}\right) \operatorname{ad}^{[1]}(\Omega) \operatorname{ad}\left(y_{2}\right) \operatorname{ad}^{[2]}(\Omega)+ \\
& 2 \operatorname{ad}^{[1]}(\Omega) \operatorname{ad}\left(y_{1}\right) \operatorname{ad}^{[2]}(\Omega) \operatorname{ad}\left(y_{2}\right) \operatorname{ad}^{[1]}(\Omega)+ \\
& \operatorname{ad}^{[2]}(\Omega) \operatorname{ad}\left(y_{1}\right) \operatorname{ad}\left(y_{2}\right) \operatorname{ad}^{[2]}(\Omega)- \\
& \operatorname{ad}^{[2]}(\Omega) \operatorname{ad}\left(y_{1}\right) \operatorname{ad}^{[1]}(\Omega) \operatorname{ad}\left(y_{2}\right) \operatorname{ad}^{[1]}(\Omega)= \\
& \operatorname{ad}^{[2]}(\Omega) \operatorname{ad}\left(y_{1}\right) \operatorname{ad}\left(y_{2}\right) \operatorname{ad}^{[2]}(\Omega),
\end{aligned}
$$

which proves the lemma.

Lemma 4.3. (1) The operations $x \rightarrow x^{2}$ and $Q$ are well defined on $J_{a}$

(2) let $f: \widetilde{L}^{m} \times \cdots \times \widetilde{L}^{m} \rightarrow \widetilde{L}^{m}$ be a homogeneous polynomial map, and let $\widetilde{f}\left(x_{1}, \cdots, x_{n}\right)$ be its full linearization. Suppose that $\tilde{f}\left(L^{m} \otimes\right.$ $\left.e_{i}+\widetilde{L}^{m} e_{i}, \widetilde{L}^{m}, \cdots, \widetilde{L}^{m}\right) \subseteq L^{m} \otimes e_{i}+\widetilde{L}^{m} e_{i}$ for all $i$.

Then, if an arbitrary value of $f$ lies in $K_{a}^{\prime}$, then an arbitrary value of $f$ lies in $K_{a}$.

Proof. (1) Choose arbitrary elements $x, y \in \widetilde{L}^{m}$ and $z^{\prime}, z \in K_{a}$. We need to show that $\left(y+z^{\prime}\right) Q(x+z)=y Q(x)$ and $(x+z)^{2}=x^{2}$. Let $x=\sum_{\pi} x_{\pi}$ be the standard decomposition of the element $x$. We have 
$z^{\prime} Q(x)=z^{\prime} \operatorname{ad}^{[2]}(a) \sum \operatorname{ad}\left(x_{\pi_{1}}\right) \operatorname{ad}\left(x_{\pi_{2}}\right)+K_{a}=0$ since $z^{\prime} \in K_{a} \subseteq K_{a}^{\prime}$. Hence $\left(y+z^{\prime}\right) Q(x+z)=y Q(x+z)$. Furthermore, it is easy to see that

$$
y Q(x+z)=y Q(x)+y \operatorname{ad}^{[2]}(a) \operatorname{ad}(x) \operatorname{ad}(z)+y Q(z) \bmod K_{a} .
$$

By Lemma 4.2, for an arbitrary standard component $x_{\pi}$ of the element $x$, we have

$$
\operatorname{ad}^{[2]}(a) \operatorname{ad}\left(x_{\pi}\right) \operatorname{ad}(z) \operatorname{ad}^{[2]}(a)=\operatorname{ad}\left(x_{\pi} \operatorname{ad}^{[2]}(a)\right) \operatorname{ad}\left(z \operatorname{ad}^{[2]}(a)\right)=0,
$$

since $z \in K_{a}^{\prime}$. Hence $y \operatorname{ad}^{[2]}(a) \operatorname{ad}\left(x_{\pi}\right) \operatorname{ad}(z) \in K_{a}$ and $y \operatorname{ad}^{[2]}(a) \operatorname{ad}(x) \operatorname{ad}(z) \in K_{a}$.

Let us show that $y Q(z)=0$. We have $z=z_{1}+\cdots+z_{s}$, where $z_{i} \in\left(L^{m} \otimes e_{i}+\widetilde{L}^{m} e_{i}\right) \cap K_{a}^{\prime}$. Let $z_{i}=\sum_{\pi} z_{i \pi}$ be the standard decomposition of the element $z_{i}$. Then $z=\sum_{\pi} z_{\pi}, z_{\pi}=\sum_{i} z_{i \pi}$, is the standard decomposition of the element $z$. Consider the family of elements $\Omega=\left\{z_{i \pi}\right\}_{i, \pi}$ and two decompositions $\Omega=\bigcup \Omega_{i}, \Omega_{i}=\left\{z_{i \pi}\right\}_{\pi}$, and $\Omega=\bigcup_{\pi} \Omega_{\pi}^{\prime}, \Omega_{\pi}^{\prime}=\left\{z_{i \pi}\right\}_{i}$. By Lemma 3.14, we have

$$
\begin{aligned}
y \operatorname{ad}^{[2]}(a) & \sum \operatorname{ad}\left(z_{\pi_{1}}\right) \operatorname{ad}\left(z_{\pi_{2}}\right) \\
& =y \operatorname{ad}^{[2]}(a) \sum_{1 \leq i<j \leq s} \operatorname{ad}\left(z_{i}\right) \operatorname{ad}\left(z_{j}\right) \quad \bmod y \operatorname{ad}^{[2]}(a) \operatorname{ad}\left(\widetilde{L}^{m}\right) .
\end{aligned}
$$

Recall that $y \operatorname{ad}^{[2]}(a) \operatorname{ad}\left(\widetilde{L}^{m}\right) \subseteq K_{a}$. The element $y \operatorname{ad}^{[2]}(a) \operatorname{ad}\left(z_{i}\right) \operatorname{ad}\left(z_{j}\right)$ lies in $L^{m} \otimes e_{i}+\widetilde{L}^{m} e_{i}$ and

$$
y \operatorname{ad}^{[2]}(a) \operatorname{ad}\left(z_{i}\right) \operatorname{ad}\left(z_{j}\right) \operatorname{ad}^{[2]}(a)=y \operatorname{ad}\left(z_{i} \operatorname{ad}^{[2]}(a)\right) \operatorname{ad}\left(z_{j} \operatorname{ad}^{[2]}(a)\right)=0
$$

by Lemma 4.2. Hence, $y \operatorname{ad}^{[2]}(a) \operatorname{ad}\left(z_{i}\right) \operatorname{ad}\left(z_{j}\right) \in K_{a}$. This implies $y Q(z)=0$.

Now let us show that $(x+z)^{2}=x^{2}$. We have $(x+z)^{2}=x^{2}+$ $a \operatorname{ad}(z) \operatorname{ad}(x)+z^{2} \bmod K_{a}$. For an arbitrary standard component $x_{\pi}$,

$$
\begin{aligned}
a \operatorname{ad}(z) \operatorname{ad}\left(x_{\pi}\right) \operatorname{ad}^{[2]}(a) & =-z \operatorname{ad}(a) \operatorname{ad}\left(x_{\pi}\right) \operatorname{ad}^{[2]}(a) \\
& =-z \operatorname{ad}^{[2]}(a) \operatorname{ad}\left(x_{\pi}\right) \operatorname{ad}(a) \\
& =0
\end{aligned}
$$

by Lemma $3.7(3)$. Hence $a \operatorname{ad}(z) \operatorname{ad}\left(x_{\pi}\right) \in K_{a}$ and $a \operatorname{ad}(z) \operatorname{ad}(x) \in K_{a}$. 
Let us show that $z^{2}=0$. We have $z^{2}=a \sum \operatorname{ad}\left(z_{\pi_{1}}\right) \operatorname{ad}\left(z_{\pi_{2}}\right)+K_{a}$. By Lemma 3.14,

$a \sum \operatorname{ad}\left(z_{\pi_{1}}\right) \operatorname{ad}\left(z_{\pi_{2}}\right)=a \sum_{1 \leq i<j \leq s} \operatorname{ad}\left(z_{i}\right) \operatorname{ad}\left(z_{j}\right) \quad \bmod a \operatorname{ad}\left(\widetilde{L}^{m}\right) \subseteq K_{a}$.

As above,

$$
\begin{aligned}
a \operatorname{ad}\left(z_{i}\right) \operatorname{ad}\left(z_{j}\right) \operatorname{ad}^{[2]}(a) & =-z_{i} \operatorname{ad}(a) \operatorname{ad}\left(z_{j}\right) \operatorname{ad}^{[2]}(a) \\
& =z_{i} \operatorname{ad}^{[2]}(a) \operatorname{ad}\left(z_{j}\right) \operatorname{ad}(a) \\
& =0
\end{aligned}
$$

by Lemma 3.7 (3). Hence $a \operatorname{ad}\left(z_{i}\right) \operatorname{ad}\left(z_{j}\right) \in K_{a}$ and $a \sum \operatorname{ad}\left(z_{\pi_{1}}\right) \operatorname{ad}\left(z_{\pi_{2}}\right) \in K_{a}$. This completes the proof of part (1). (2) Now let $f: \widetilde{L}^{m} \times \cdots \times \widetilde{L}^{m} \rightarrow \widetilde{L}^{m}$ be a homogeneous polynomial map with the full linearization $\tilde{f}$. By Lemma 3.8 (2), for polynomial maps, the $F$-linear span of all values of $f$ is equal to the $F$-linear span of all values of $\tilde{f}$. Hence, we need to show that $\widetilde{f}\left(\widetilde{L}^{m}, \cdots, \widetilde{L}^{m}\right) \subseteq K_{a}$. Since $\widetilde{L}^{m}=\sum_{i}\left(L^{m} \otimes e_{i}+\widetilde{L}^{m} e_{i}\right)$, it follows that $\widetilde{f}\left(\widetilde{L}^{m}, \cdots, \widetilde{L}^{m}\right)=$ $\sum_{i} \tilde{f}\left(L^{m} \otimes e_{i}+\widetilde{L}^{m} e_{i}, \widetilde{L}^{m}, \cdots, \widetilde{L}^{m}\right)$. By our assumption, $\widetilde{f}\left(L^{m} \otimes e_{i}+\right.$ $\left.\widetilde{L}^{m} e_{i}, \widetilde{L}^{m}, \cdots, \widetilde{L}^{m}\right) \subseteq K_{a}^{\prime} \cap\left(L^{m} \otimes e_{i}+\widetilde{L}^{m} e_{i}\right) \subseteq K_{a}$. This completes the proof of assertion (2).

The following proposition is a linearized an quadratic version of the construction in [FLGGL].

Proposition 4.4. $J_{a}=\left(J_{a}, x \rightarrow x^{2}, Q\right)$ is a quadratic Jordan algebra.

Since the ground field is infinite partial linearizations of the identities (M1)-(M6) follows from these identities (see [Jac1], [ZSSS]).

We will translate the identities (M1)-(M6) into the language of Lie algebras. The identities (M1)-(M6) translate as

(M1) $x \operatorname{ad}^{[2]}(a) \operatorname{ad}(x) \operatorname{ad}(y)=a \operatorname{ad}\left(a \operatorname{ad}^{[2]}(x)\right) \operatorname{ad}(y) \bmod K_{a}$,

(M2) $y \operatorname{ad}^{[2]}(a) \operatorname{ad}^{[2]}(x) \operatorname{ad}(a) \operatorname{ad}(x)=-a \operatorname{ad}(x) \operatorname{ad}(y) \operatorname{ad}^{[2]}(a) \operatorname{ad}^{[2]}(x)$ $=y \operatorname{ad}([a, x]) \operatorname{ad}^{[2]}(a) \operatorname{ad}^{[2]}(x) \bmod K_{a}$,

(M3) $a \operatorname{ad}^{[2]}(x) \operatorname{ad}^{[2]}(a) \operatorname{ad}^{[2]}(x)=a \operatorname{ad}^{[2]}\left(a \operatorname{ad}^{[2]}(x)\right) \bmod K_{a}$,

(M4) $a \operatorname{ad}^{[2]}(x) \operatorname{ad}^{[2]}(a) \operatorname{ad}^{[2]}(y) \operatorname{ad}^{[2]}(a) \operatorname{ad}^{[2]}(x)$

$=a \operatorname{ad}^{[2]}\left(y \operatorname{ad}^{[2]}(a) \operatorname{ad}^{[2]}(x)\right) \bmod K_{a}$, 
$(\mathrm{M} 5) y \operatorname{ad}^{[2]}(a) \operatorname{ad}^{[2]}\left(a \operatorname{ad}^{[2]}(x)\right)=y \operatorname{ad}^{[2]}(a) \operatorname{ad}^{[2]}(x) \operatorname{ad}^{[2]}(a) \operatorname{ad}^{[2]}(x)$ $\bmod K_{a}$,

(M6) $z \operatorname{ad}^{[2]}(a) \operatorname{ad}^{[2]}\left(y \operatorname{ad}^{[2]}(a) \operatorname{ad}^{[2]}(x)\right)$ $=z \operatorname{ad}^{[2]}(a) \operatorname{ad}^{[2]}(x) \operatorname{ad}^{[2]}(a) \operatorname{ad}^{[2]}(y) \operatorname{ad}^{[2]}(a) \operatorname{ad}^{[2]}(x) \bmod K_{a}$.

Remark. In the formulas above, we have operators $\operatorname{ad}^{[2]}(x)$, $\operatorname{ad}^{[2]}\left(a \operatorname{ad}^{[2]}(x)\right), \operatorname{ad}^{[2]}\left(y \operatorname{ad}^{[2]}(a) \operatorname{ad}^{[2]}(x)\right)$ acting on elements from the space $F a+\widetilde{L^{m}}$ ad $^{[2]}(a)$. In the definition of Jordan operations on $J_{a}=\widetilde{L^{m}} / K_{a}$ above, we noticed that $\left(F a+\widetilde{L^{m}} \operatorname{ad}^{[2]}(a)\right)$ ad $\left(\widetilde{L^{m}}\right) \subseteq K_{a}$. Hence for an arbitrary element $u \in\left\{x, a \operatorname{ad}^{[2]}(x), y \operatorname{ad}^{[2]}(a) \operatorname{ad}^{[2]}(x)\right\}$, the operator $\operatorname{ad}^{[2]}(u)$ is understood as $\sum \operatorname{ad}\left(u_{i}\right) \operatorname{ad}\left(u_{j}\right)$, where $u=\sum u_{j}$ is the standard decomposition, the sum runs over all 2 -element sets $(i, j)$ and the order of factors in $\operatorname{ad}\left(u_{i}\right) \operatorname{ad}\left(u_{j}\right)$ is irrelevant modulo $K_{a}$.

Let $x, y \in \widetilde{L^{m}} ; x=\sum_{\pi} x_{\pi}, y=\sum_{\tau} y_{\tau}$ the standard decompositions. At first, we will prove the identities (M1)-(M6) under the additional assumption that $\left[x_{\pi_{i}}, x_{\pi_{j}}\right]=\left[y_{\tau_{i}}, y_{\tau_{j}}\right]=0$,

$$
\operatorname{ad}\left(x_{\pi_{i}}\right) \operatorname{ad}\left(x_{\pi_{j}}\right) \operatorname{ad}\left(x_{\pi_{k}}\right)=\operatorname{ad}\left(y_{\tau_{1}}\right) \operatorname{ad}\left(y_{\tau_{2}}\right) \operatorname{ad}\left(y_{\tau_{3}}\right)=0
$$

for all $i, j, k$.

More precisely, let $L_{0}^{\prime}$ be the Lie algebra presented by generators $a_{1}, \cdots, a_{n}, x_{1}, \cdots, x_{s}, y_{1}, \cdots, y_{t}$ and the following relations:

$$
\left[\operatorname{Id}\left(a_{i}\right), \operatorname{Id}\left(a_{i}\right)\right]=\left[\operatorname{Id}\left(x_{j}\right), \operatorname{Id}\left(x_{j}\right)\right]=\left[\operatorname{Id}\left(y_{k}\right), \operatorname{Id}\left(y_{k}\right)\right]=(0),
$$

where $\operatorname{Id}\left(a_{i}\right), \operatorname{Id}\left(x_{j}\right), \operatorname{Id}\left(y_{k}\right)$ denote the ideals generated by $a_{i}, x_{j}, y_{k}$ respectively, $1 \leq i \leq n, 1 \leq j \leq s, 1 \leq k \leq t ;\left[a_{i}, a_{j}\right]=0,1 \leq i, j \leq n$; the operators $\operatorname{ad}^{[k]}(a)=\sum \operatorname{ad}\left(a_{i_{1}}\right) \cdots \operatorname{ad}\left(a_{i_{k}}\right)$, where the sum is taken over all $k$-element subsets of $\{1,2, \cdots, n\}$ is equal to 0 for $k \geq 3$.

$$
\text { Denote } a=\sum_{i=1}^{n} a_{i}, x=\sum_{j=1}^{s} x_{j} .
$$

Remark. The generators $a_{1}, \cdots, a_{n}$ should not be confused with elements $a_{1}, \cdots, a_{r} \in \widetilde{L^{m}}$ used to define $a^{\prime}=w^{\prime}\left(a_{1}, \cdots, a_{r-1}\right)$, $a=w\left(a_{1}, \cdots, a_{r}\right)$ above.

In the algebra $L_{0}^{\prime}$, define linear operators

$$
\operatorname{ad}^{[2]}(a)=\sum_{1 \leq i<j \leq n} \operatorname{ad}\left(a_{i}\right) \operatorname{ad}\left(a_{j}\right),
$$




$$
\begin{gathered}
\operatorname{ad}^{[2]}(x)=\sum_{1 \leq i<j \leq s} \operatorname{ad}\left(x_{i}\right) \operatorname{ad}\left(x_{j}\right), \\
\operatorname{ad}^{[2]}(y)=\sum_{1 \leq i<j \leq t} \operatorname{ad}\left(y_{i}\right) \operatorname{ad}\left(y_{j}\right), \\
\operatorname{ad}^{[2]}\left(a \operatorname{ad}^{[2]}(x)\right)=\sum_{1 \leq i<j \leq n} \operatorname{ad}\left(a_{i} \operatorname{ad}^{[2]}(x)\right) \operatorname{ad}\left(a_{j} \operatorname{ad}^{[2]}(x)\right), \\
\operatorname{ad}^{[2]}\left(y \operatorname{ad}^{[2]}(a) \operatorname{ad}^{[2]}(x)\right)=\sum_{1 \leq i<j \leq t} \operatorname{ad}\left(y_{i} \operatorname{ad}^{[2]}(a) \operatorname{ad}^{[2]}(x)\right) \operatorname{ad}\left(y_{j} \operatorname{ad}^{[2]}(a) \operatorname{ad}^{[2]}(x)\right)
\end{gathered}
$$

and consider the elements

$\left(\mathrm{M1}^{\prime}\right)\left(x \operatorname{ad}^{[2]}(a) \operatorname{ad}(x) \operatorname{ad}(y)-a \operatorname{ad}\left(a \operatorname{ad}^{[2]}(x)\right) \operatorname{ad}(y) \operatorname{ad}^{[2]}(a)\right.$,

$\left(\mathrm{M}^{\prime}\right)\left(y \operatorname{ad}^{[2]}(a) \operatorname{ad}^{[2]}(x) \operatorname{ad}(a) \operatorname{ad}(x)\right.$

$$
\left.+a \operatorname{ad}(x) \operatorname{ad}(y) \operatorname{ad}^{[2]}(a) \operatorname{ad}^{[2]}(x)\right) \operatorname{ad}^{[2]}(a) \text {, }
$$

$\left(\mathrm{M}^{\prime}\right)\left(a \operatorname{ad}^{[2]}(x) \operatorname{ad}^{[2]}(a) \operatorname{ad}^{[2]}(x)-a \operatorname{ad}^{[2]}\left(a \operatorname{ad}^{[2]}(x)\right)\right) \operatorname{ad}^{[2]}(a)$,

$\left(\mathrm{M}^{\prime}\right)\left(a \operatorname{ad}^{[2]}(x) \operatorname{ad}^{[2]}(a) \operatorname{ad}^{[2]}(y) \operatorname{ad}^{[2]}(a) \operatorname{ad}^{[2]}(x)\right.$

$\left.-a \operatorname{ad}^{[2]}\left(y \operatorname{ad}^{[2]}(a) \operatorname{ad}^{[2]}(x)\right)\right) \operatorname{ad}^{[2]}(a)$,

$\left(\mathrm{M5}^{\prime}\right)\left(y \operatorname{ad}^{[2]}(a) \operatorname{ad}^{[2]}\left(a \operatorname{ad}^{[2]}(x)\right)\right.$

$\left.-y \operatorname{ad}^{[2]}(a) \operatorname{ad}^{[2]}(x) \operatorname{ad}^{[2]}(a) \operatorname{ad}^{[2]}(x)\right) \operatorname{ad}^{[2]}(a)$,

$\left(\mathrm{M6}^{\prime}\right)\left(z \operatorname{ad}^{[2]}(a) \operatorname{ad}^{[2]}\left(y \operatorname{ad}^{[2]}(a) \operatorname{ad}^{[2]}(x)\right)\right.$

$-z \operatorname{ad}^{[2]}(a) \operatorname{ad}^{[2]}(x) \operatorname{ad}^{[2]}(a) \operatorname{ad}^{[2]}(y) \operatorname{ad}^{[2]}(a) \operatorname{ad}^{[2]}(x) \operatorname{ad}^{[2]}(a)$.

Now, consider the Lie algebra $L_{0}$ that is obtained from $L_{0}^{\prime}$ by imposing additional relations:

$\left[x_{i}, x_{j}\right]=0,1 \leq i, j \leq s ;\left[y_{i}, y_{j}\right]=0,1 \leq i, j \leq t ;\left[L_{0}, x_{i_{1}}, x_{i_{2}}, x_{i_{3}}\right]=$ $\left[L_{0}, y_{j_{1}}, y_{j_{2}}, y_{j_{3}}\right]=(0)$, for all $1 \leq i_{1}, i_{2}, i_{3} \leq s, 1 \leq j_{1}, j_{2}, j_{3} \leq t$.

We will show that the elements $\left(\mathrm{M}^{\prime}\right)-\left(\mathrm{M} 6^{\prime}\right)$ are equal to zero in the Lie algebra $L_{0}$.

Lemma 4.5. $\left[a \operatorname{ad}^{[2]}(x), a\right]+\left[x \operatorname{ad}^{[2]}(a), x\right] \in\left[L_{0}, a, a\right]$.

Proof. If $p \neq 2$ then $\operatorname{ad}^{[2]}(x)=\frac{1}{2} \operatorname{ad}(x)^{2}, \operatorname{ad}^{[2]}(a)=\frac{1}{2} \operatorname{ad}^{2}(a)$, which makes the assertion of the lemma obvious.

Let $p=2$. Denote $a^{\prime}=a_{i}, a^{\prime \prime}=a_{j}, x^{\prime}=x_{k}, x^{\prime \prime}=x_{e}$. We will show that

$$
\left[a^{\prime}, x^{\prime}, x^{\prime \prime}, a^{\prime \prime}\right]+\left[a^{\prime \prime}, x^{\prime}, x^{\prime \prime}, a^{\prime}\right]=\left[x^{\prime}, a^{\prime}, a^{\prime \prime}, x^{\prime \prime}\right]+\left[x^{\prime \prime}, a^{\prime}, a^{\prime \prime}, x^{\prime}\right] .
$$


Indeed, $\left[a^{\prime}, x^{\prime}, x^{\prime \prime}, a^{\prime \prime}\right]+\left[a^{\prime \prime}, x^{\prime}, x^{\prime \prime}, a^{\prime}\right]=\left[\left[a^{\prime}, x^{\prime}\right],\left[x^{\prime \prime}, a^{\prime \prime}\right]\right]+\left[a^{\prime}, x^{\prime}, a^{\prime \prime}, x^{\prime \prime}\right]+$ $\left[\left[a^{\prime \prime}, x^{\prime}\right],\left[x^{\prime \prime}, a^{\prime}\right]\right]+\left[a^{\prime \prime}, x^{\prime}, a^{\prime}, x^{\prime \prime}\right]=\left[\left[a^{\prime}, x^{\prime}\right],\left[x^{\prime \prime}, a^{\prime \prime}\right]\right]+\left[\left[a^{\prime \prime}, x^{\prime}\right],\left[x^{\prime \prime}, a^{\prime}\right]\right]$, since $\left[a^{\prime}, x^{\prime}, a^{\prime \prime}\right]+\left[a^{\prime \prime}, x^{\prime}, a^{\prime}\right]=\left[\left[a^{\prime}, a^{\prime \prime}\right], x^{\prime}\right]=0$.

Similarly,

$$
\left[x^{\prime}, a^{\prime}, a^{\prime \prime}, x^{\prime \prime}\right]+\left[x^{\prime \prime}, a^{\prime}, a^{\prime \prime}, x^{\prime}\right]=\left[\left[x^{\prime}, a^{\prime}\right],\left[a^{\prime \prime}, x^{\prime \prime}\right]\right]+\left[\left[x^{\prime \prime}, a^{\prime}\right],\left[a^{\prime \prime}, x^{\prime}\right]\right],
$$

which finishes the proof of the lemma.

Now (M1') immediately follows from Lemma 4.5 since $L_{0} \operatorname{ad}(a)^{2} \operatorname{ad}(y) \subseteq K_{a}$. The latter inclusion follows from the following argument. The equality (see Lemma $3.7(3)$ )

$$
\begin{aligned}
0= & \operatorname{ad}\left(y \operatorname{ad}^{[4]}(a)\right) \\
= & \operatorname{ad}(y) \operatorname{ad}^{[4]}(a)-\operatorname{ad}(a) \operatorname{ad}(y) \operatorname{ad}^{[3]}(a)+\operatorname{ad}^{[2]}(a) \operatorname{ad}(y) \operatorname{ad}^{[2]}(a) \\
& -\operatorname{ad}^{[3]}(a) \operatorname{ad}(y) \operatorname{ad}(a)+\operatorname{ad}^{[4]}(a) \operatorname{ad}(y)
\end{aligned}
$$

implies $\operatorname{ad}^{[2]}(a) \operatorname{ad}(y) \operatorname{ad}^{[2]}(a)=0$. Hence,

$$
L_{0} \operatorname{ad}(a)^{2} \operatorname{ad}(y) \operatorname{ad}^{[2]}(a) \subseteq L_{0} \operatorname{ad}^{[2]}(a) \operatorname{ad}(y) \operatorname{ad}^{[2]}(a)=0 .
$$

Let us prove $\left(\mathrm{M}^{\prime}\right)$. From $\operatorname{ad}^{[2]}(x) \operatorname{ad}(a) \operatorname{ad}(x)=\operatorname{ad}(x) \operatorname{ad}(a) \operatorname{ad}^{[2]}(x)$ and $\operatorname{ad}^{[2]}(a) \operatorname{ad}(x) \operatorname{ad}(a)=\operatorname{ad}(a) \operatorname{ad}(x) \operatorname{ad}^{[2]}(a)$ (see Lemma 3.7 (3)), it follows that

$$
\begin{aligned}
& y \operatorname{ad}^{[2]}(a) \operatorname{ad}^{[2]}(x) \operatorname{ad}(a) \operatorname{ad}(x)= \\
& y \operatorname{ad}^{[2]}(a) \operatorname{ad}(x) \operatorname{ad}(a) \operatorname{ad}^{[2]}(x)= \\
& y \operatorname{ad}(a) \operatorname{ad}(x) \operatorname{ad}^{[2]}(a) \operatorname{ad}^{[2]}(x)= \\
& y \operatorname{ad}([a, x]) \operatorname{ad}^{[2]}(a) \operatorname{ad}^{[2]}(x),
\end{aligned}
$$

since $\operatorname{ad}(a) \operatorname{ad}^{[2]}(a)=3 \operatorname{ad}^{[3]}(a)=0$.

Now we will prove $\left(\mathrm{M3}^{\prime}\right)$. We have

$$
\operatorname{ad}^{[2]}\left(a \operatorname{ad}^{[2]}(x)\right)=\sum \operatorname{ad}\left(a_{i} \operatorname{ad}^{[2]}(x)\right) \operatorname{ad}\left(a_{j} \operatorname{ad}^{[2]}(x)\right),
$$

where the sum is taken over all 2-element subsets $(i, j)$. By applying Lemma 4.2 to $\Omega=\left\{x_{1}, \cdots, x_{m}\right\}, y_{1}=a_{i}, y_{2}=a_{j}$, we get

$$
\operatorname{ad}\left(a_{i} \operatorname{ad}^{[2]}(x)\right) \operatorname{ad}\left(a_{j} \operatorname{ad}^{[2]}(x)\right)=\operatorname{ad}^{[2]}(x) \operatorname{ad}\left(a_{i}\right) \operatorname{ad}\left(a_{j}\right) \operatorname{ad}^{[2]}(x) .
$$


Hence,

$$
\begin{aligned}
& \sum \operatorname{ad}\left(a_{i} \operatorname{ad}^{[2]}(x)\right) \operatorname{ad}\left(a_{j} \operatorname{ad}^{[2]}(x)\right)= \\
& \operatorname{ad}^{[2]}(x)\left(\sum \operatorname{ad}\left(a_{i}\right) \operatorname{ad}\left(a_{j}\right)\right) \operatorname{ad}^{[2]}(x)= \\
& \operatorname{ad}^{[2]}(x) \operatorname{ad}^{[2]}(a) \operatorname{ad}^{[2]}(x)
\end{aligned}
$$

as claimed.

Let us prove $\left(\mathrm{M} 4^{\prime}\right)$. We have

$$
\begin{aligned}
& \operatorname{ad}^{[2]}\left(y \operatorname{ad}^{[2]}(a) \operatorname{ad}^{[2]}(x)\right)= \\
& \sum \operatorname{ad}\left(y_{i} \operatorname{ad}^{[2]}(a) \operatorname{ad}^{[2]}(x)\right) \operatorname{ad}\left(y_{j} \operatorname{ad}^{[2]}(a) \operatorname{ad}^{[2]}(x)\right) .
\end{aligned}
$$

By Lemma 4.2, with $\Omega=\left\{x_{1}, \cdots, x_{m}\right\}$, we get

$$
\begin{aligned}
& \operatorname{ad}\left(y_{i} \operatorname{ad}^{[2]}(a) \operatorname{ad}^{[2]}(x)\right) \operatorname{ad}\left(y_{j} \operatorname{ad}^{[2]}(a) \operatorname{ad}^{[2]}(x)\right)= \\
& \operatorname{ad}^{[2]}(x) \operatorname{ad}\left(y_{i} \operatorname{ad}^{[2]}(a)\right) \operatorname{ad}\left(y_{j} \operatorname{ad}^{[2]}(a)\right) \operatorname{ad}^{[2]}(x) .
\end{aligned}
$$

Again, by Lemma 4.2 with $\Omega=\left\{a_{1}, \cdots, a_{n}\right\}$

$$
\operatorname{ad}\left(y_{i} \operatorname{ad}^{[2]}(a)\right) \operatorname{ad}\left(y_{j} \operatorname{ad}^{[2]}(a)\right)=\operatorname{ad}^{[2]}(a) \operatorname{ad}\left(y_{i}\right) \operatorname{ad}\left(y_{j}\right) \operatorname{ad}^{[2]}(a) .
$$

Finally, we get

$$
\operatorname{ad}^{[2]}\left(y \operatorname{ad}^{[2]}(a) \operatorname{ad}^{[2]}(x)\right)=\operatorname{ad}^{[2]}(x) \operatorname{ad}^{[2]}(a) \operatorname{ad}^{[2]}(y) \operatorname{ad}^{[2]}(a) \operatorname{ad}^{[2]}(x),
$$

as claimed.

We will now prove $\left(\mathrm{M}^{\prime}\right)$. We have already shown above that by Lemma 4.2, we have

$$
\operatorname{ad}^{[2]}\left(a \operatorname{ad}^{[2]}(x)\right)=\operatorname{ad}^{[2]}(x) \operatorname{ad}^{[2]}(a) \operatorname{ad}^{[2]}(x),
$$

which implies the claim.

To prove $\left(\mathrm{M} 6^{\prime}\right)$, we need only to recall the equality

$$
\operatorname{ad}^{[2]}\left(y \operatorname{ad}^{[2]}(a) \operatorname{ad}^{[2]}(x)\right)=\operatorname{ad}^{[2]}(x) \operatorname{ad}^{[2]}(a) \operatorname{ad}^{[2]}(y) \operatorname{ad}^{[2]}(a) \operatorname{ad}^{[2]}(x)
$$

that was proved above.

Since the elements $\left(\mathrm{M}^{\prime}\right)-\left(\mathrm{M} 6^{\prime}\right)$ are equal to zero in $L_{0}$, it follows that in the algebra $L_{0}^{\prime}$, the elements of $\left(\mathrm{M}^{\prime}\right)-\left(\mathrm{M} 6^{\prime}\right)$ are linear combinations of

(1) expressions in $x_{i}$ 's,$y_{j}$ 's, $z, a_{1}, \cdots, a_{n}$ involving at least one commutator $\left[x_{i}, x_{j}\right], 1 \leq i, j \leq s$ or $\left[y_{i}, y_{j}\right], 1 \leq i, j \leq t$, 
(2) expressions involving $\operatorname{ad}\left(x_{i_{1}}\right) \operatorname{ad}\left(x_{i_{2}}\right) \operatorname{ad}\left(x_{i_{3}}\right) \operatorname{or} \operatorname{ad}\left(y_{j_{1}}\right) \operatorname{ad}\left(y_{j_{2}}\right) \operatorname{ad}\left(y_{j_{3}}\right)$, $1 \leq i_{1}, i_{2}, i_{3} \leq s, 1 \leq j_{1}, j_{2}, j_{3} \leq t$.

Moreover, since the relations of the algebra $L_{0}^{\prime}$ are homogeneous in $x_{i}$ 's, $y_{j}$ 's, and in the total number of generators $a_{1}, \cdots, a_{n}$, it follows that the presentations of $\left(\mathrm{M}^{\prime}\right)-\left(\mathrm{M} 6^{\prime}\right)$ as linear combinations of (1), (2) preserve the degrees in $x_{i}{ }^{\prime}$ s, $y_{j}{ }^{\prime}$ s, and the total degree in $a_{1}, \cdots, a_{n}$.

Now we consider arbitrary elements $x, y \in \widetilde{L^{m}}$ and drop the assumptions on components of standard decompositions of $x, y$. Let $x=x_{\pi_{1}}+\cdots+x_{\pi_{s}}, y=y_{\tau_{1}}+\cdots+y_{\tau_{s}}, a_{r}=a_{r 1}+\cdots+a_{r n}$ be the standard decompositions of $x, y, a_{r}$ respectively. Then $a=\sum_{i=1}^{n} a_{i}$, where $a_{i}=a_{r i} \operatorname{ad}^{[2]}\left(a^{\prime}\right)$.

The mapping $a_{i} \rightarrow a_{r i} \operatorname{ad}^{[2]}\left(a^{\prime}\right), x_{j} \rightarrow x_{\pi_{j}}, y_{k} \rightarrow y_{\tau_{k}}, 1 \leq i \leq$ $n, 1 \leq j \leq s, 1 \leq k \leq t$, extends to a homomorphism $L_{0}^{\prime} \rightarrow$ $\widetilde{L^{m}}$. Moreover, the operators $\operatorname{ad}^{[2]}(a), \operatorname{ad}^{[2]}(x), \operatorname{ad}^{[2]}(y), \operatorname{ad}^{[2]}\left(a \operatorname{ad}^{[2]}(x)\right)$, $\operatorname{ad}^{[2]}\left(y \operatorname{ad}^{[2]}(a) \operatorname{ad}^{[2]}(x)\right)$ project to the similar operators on $\widetilde{L^{m}}$, by Lemma 3.14 .

Hence, the elements $\left(\mathrm{M}^{\prime}\right)-\left(\mathrm{M} 6^{\prime}\right)$ of $\widetilde{L^{m}}$ are linear combinations of

(1) expressions in $x_{\pi_{i}}, y_{\tau_{j}}, a_{1}, \cdots, a_{n}$ involving at least one commutator $\left[x_{\pi_{i}}, x_{\pi_{j}}\right]$ or $\left[y_{\tau_{i}}, y_{\tau_{j}}\right]$

(2) expressions involving $\operatorname{ad}\left(x_{\pi_{i}}\right) \operatorname{ad}\left(x_{\pi_{j}}\right) \operatorname{ad}\left(x_{\pi_{k}}\right)$ or $\operatorname{ad}\left(y_{\tau_{i}}\right) \operatorname{ad}\left(y_{\tau_{j}}\right) \operatorname{ad}\left(y_{\tau_{k}}\right)$.

These presentations, as linear combinations of (1) and (2), preserve the degrees in $x_{\pi_{i}}$ 's, $y_{\tau_{j}}$ 's and the total degree in $a_{1}, \cdots, a_{n}$.

Replacing $\operatorname{ad}\left(a_{i}\right)$ in these expressions by

$\operatorname{ad}\left(a_{r i} \operatorname{ad}^{[2]}\left(a^{\prime}\right)\right)=\operatorname{ad}\left(a_{r i}\right) \operatorname{ad}^{[2]}\left(a^{\prime}\right)-\operatorname{ad}\left(a^{\prime}\right) \operatorname{ad}\left(a_{r i}\right) \operatorname{ad}\left(a^{\prime}\right)+\operatorname{ad}^{[2]}\left(a^{\prime}\right) \operatorname{ad}\left(a_{r i}\right)$

we get expressions whose degree in $a^{\prime}$ exceeds the total degree in the other variables $x_{\pi_{i}}, y_{\tau_{j}}, z, a_{r i}$ by 1 . In case (1), we merge two elements $x_{\pi_{i}}, x_{\pi_{j}}$ or $y_{\tau_{i}}, y_{\tau_{j}}$ together. Hence, the degree in $a^{\prime}$ exceeds the total degree in the other elements by 2 . The only property of the element $a$ that was used in Lemma 4.1 was $\operatorname{ad}^{[k]}(a)=0$ for $k \geq 3$. We have $\operatorname{ad}^{[k]}\left(a^{\prime}\right)=0, k \geq 3$. Hence, we can apply Lemma 4.1 to the element $a^{\prime}$. By Lemma 4.1 (1), these expressions are equal to zero. In case (2), 
we only need to refer to Lemma 4.1 (3). We proved that the expressions $\left(\mathrm{M}^{\prime}\right)-\left(\mathrm{M} 6^{\prime}\right)$ are equal to 0 , which means that the expressions (M1)-(M6) are equal to 0 modulo $K_{a}^{\prime}$. By Lemma 4.3 (2), they are equal to 0 modulo $K_{a}$, which finishes the proof of Proposition 4.4.

Let us consider basic examples of quadratic Jordan algebras.

Example 1. Let $A$ be an associative algebra. Let $y Q(x)=x y x ; x, y \in$ $A$. Then the vector space $A$ with the operators $x \rightarrow x^{2}$ and $x \rightarrow Q(x)$ is a quadratic Jordan algebra, which is denoted as $A^{(+)}$.

Example 2. Let $A$ be an associative algebra with an involution $*$ : $A \rightarrow A$. Then $H(A, *)=\left\{a \in A \mid a^{*}=a\right\}$ is a subalgebra of the quadratic Jordan algebra $A^{(+)}$.

Example 3. Let $V$ be a vector space and let $q: V \rightarrow F$ be a quadratic form with the associated bilinear form $q(v, w)=q(v+w)-q(v)-q(w)$. Fix an element of $V$ that we will denote as $\mathbb{1}$ (a base point) such that $q(\mathbb{1})=1$. For arbitrary elements $v, w \in V$ define

$$
v^{2}=q(v, \mathbb{1}) v-q(v) \mathbb{1}, w Q(v)=q(v, \bar{w}) v-q(v) \bar{w}
$$

where $\bar{w}=q(w, \mathbb{1}) \mathbb{1}-w$. These equations make $V$ a quadratic Jordan algebra. We will denote it as $J(q, \mathbb{1})$.

Example 4. Albert algebras of a nondegenerate admissible cubic form on a 27-dimensional space (see [Jac1, Jac4]).

Powers of elements in a quadratic Jordan algebra $J$ are defined inductively: we define $x^{1}=x$; for an even $n=2 k$ we define $x^{n}=\left(x^{k}\right)^{2}$; and for an odd $n=2 k+1$ we define $x^{n}=x Q\left(x^{k}\right)$. For arbitrary integers $i \geq 0, j \geq 0, k \geq 0$ we have $x^{i} Q\left(x^{j}\right)=x^{i+2 j}, x^{i} \circ x^{j}=$ $2 x^{i+j},\left\{x^{i}, x^{j}, x^{k}\right\}=2 x^{i+j+k}$.

A quadratic Jordan algebra $J$ is said to be nil of bounded degree $n$ if $x^{n}=0$ for an arbitrary element $x \in J$ and if $n$ is a minimal integer with this property.

Just as in $\S 2$ we call an element of the free quadratic Jordan algebra $F J\langle X\rangle$ an $S$-identity if it lies in the kernel of the homomorphism $F J\langle X\rangle \rightarrow F\langle X\rangle^{(+)}, x \rightarrow x$, where $F\langle X\rangle$ is the free associative algebra. 
We say that a quadratic Jordan algebra $J$ is PI if there exists an element $f\left(x_{1}, \cdots, x_{r}\right) \in F J\langle X\rangle$ that is not an $S$-identity and that is identically zero on $J$.

In this paper, we call an element $a$ of a quadratic Jordan algebra an absolute zero divisor if $Q(a)=0$. This terminology is not standard. (In the standard terminology, we should have also assumed $a \neq 0$ and $a^{2}=$ 0.) A quadratic Jordan algebra that does not contain nonzero absolute zero divisors is called nondegenerate. The smallest ideal $M(J)$ of a Jordan algebra $J$ such that the factor algebra $J / M(J)$ is nondegenerate is called the McCrimmon radical of the algebra $J$. The McCrimmon radical of an arbitrary quadratic Jordan algebra lies in the nil radical $\operatorname{Nil}(J)([$ Zel1, $\mathrm{McC} 2])$.

A nondegenerate quadratic Jordan algebra is said to be nondegenerate prime if two arbitrary nonzero ideals of $J$ have nonzero intersection.

In [Zel3, The it is shown that an arbitrary nondegenerate Jordan algebra is a subdirect product of nondegenerate prime Jordan algebras.

Let $\operatorname{Sym}_{n}\left(x_{1}, \cdots, x_{n}\right)$ be the full linearization of $x_{1}^{n}$ in the free Jordan algebra $F J\langle X\rangle$.

Lemma 4.6. There exists a function $d: \mathbb{N} \rightarrow \mathbb{N}$ such that an arbitrary nondegenerate prime quadratic Jordan algebra over a field of characteristic $p>0$ satisfying a PI of degree $n$ satisfies the identity $\operatorname{Sym}_{d(n)}\left(x_{1}, \cdots, x_{d(n)}\right)=0$.

Proof. Let us notice first that if $J$ is a quadratic Jordan algebra of dimension $d$ then $J$ satisfies the identity $\operatorname{Sym}_{d(p-1)+1}\left(x_{1}, \cdots, x_{d(p-1)+1}\right)=$ 0 . Indeed, if $e_{1}, \cdots, e_{d}$ is a basis of $J$ then among any $d(p-1)+1$ elements from $\left\{e_{1}, \cdots, e_{d}\right\}$ at least $p$ elements are equal. This implies the claim.

In [MZ it was shown that if $J$ is a nondegenerate prime quadratic Jordan algebra, then one of the following possibilities holds:

(1) there exists a prime associative algebra $A$ such that

$$
A^{(+)} \subseteq J \subseteq \mathbb{Q}(A)^{(+)},
$$

where $\mathbb{Q}(A)$ is the Martindale ring of the quotients of $A$ (see $[\mathrm{Mar}]$ ); 
(2) there exists a prime associative algebra $A$ with an involution $*: A \rightarrow A$, such that

$$
H\left(A_{0}, *\right) \subseteq J \subseteq H(\mathbb{Q}(A), *)
$$

where $A_{0}$ is the subalgebra of $A$ generated by elements $a+a^{*}, a a^{*}$, $a \in A$, and $\mathbb{Q}(A)$ is the Martindale ring of quotients of the algebra $A$ (see $\mathrm{Mar})$;

(3) $J$ is a form of an exceptional 27-dimensional Albert algebra over a field $F$;

(4) $J$ is embeddable in a quadratic Jordan algebra $J\left(q, v_{0}\right)$ of a nondegenerate quadratic form $q$ with a basepoint $v_{0}$ in a vector space over some extension of the base field $F$.

If $A^{(+)} \subseteq J \subseteq \mathbb{Q}(A)^{(+)}$, then $A$ is a prime associative algebra satisfying an identity of degree $n$. Hence the center $Z(A)$ of $A$ is nonzero and the algebra $\mathbb{Q}(A)=(Z(A) \backslash\{0\})^{-1} A$ is of dimension $\leq\left[\frac{n}{2}\right]^{2}$ over the field $K=(Z(A) \backslash\{0\})^{-1} Z(A)$ (see [Row $]$ ). Hence the algebra $\mathbb{Q}(A)$ satisfies the identity $\operatorname{Sym}_{\left[\frac{n}{2}\right]^{2}(p-1)+1}=0$.

Suppose that $H\left(A_{0}, *\right) \subseteq J \subseteq H(\mathbb{Q}(A)$,*). S. Amitsur Ami] proved that there exists a function $h(n)$ with the following property:

if an involutive associative algebra satisfies an identity of degree $n$ with an involution then it satisfies an identity of degree $\leq h(n)$. As we have shown above, the algebra $\mathbb{Q}(A)$ in this case has dimension $\leq\left[\frac{h(n)}{2}\right]^{2}$ over its center and satisfies the identity $\operatorname{Sym}_{\left[\frac{h(n)}{2}\right]^{2}(p-1)+1}=0$.

The same argument applies to case (3): the algebra $J$ satisfies the identity $\operatorname{Sym}_{27(p-1)+1}=0$.

Consider now the quadratic Jordan algebra $J$ of a quadratic form $q$ on a vector space $V$ where $v_{0} \in V$ is a basepoint. The quadratic form $q$ can be extended to the scalar product $V \otimes_{F} \widehat{E}, \widehat{E}=E+F \cdot 1$. For an arbitrary element $u \in V \otimes_{F} E$ we have $u^{2}=q\left(u, v_{0}\right) u-q(u) v_{0}$. The elements $a=q\left(u, v_{0}\right), b=q(u)$ lie in $E$. Hence $a^{p}=b^{p}=0$. For an arbitrary $k \geq 1$ we have

$$
u^{2 k}=\sum_{i+j=k} a^{i} b^{j} u_{i j}, u_{i j} \in V \otimes_{F} \widehat{E} .
$$

Hence $u^{2(2 p-1)}=0$. This implies that the algebra $J$ satisfies the identity $\mathrm{Sym}_{4 p-2}=0$ and finishes the proof of the lemma. 
Lemma 4.7. Let $J$ be a quadratic Jordan F-algebra that satisfies the identity $x^{n}=0, n \geq 2$. Then,

(1) for an arbitrary element $a \in J$, the elements $a^{n+1}, a^{n+2}, \cdots, a^{2 n-1}$ are absolute zero divisiors of $J$;

(2) if $J$ satisfies the identities $x^{n}=x^{n+1}=\cdots=x^{2 n-1}=0$, then for an arbitrary $a \in J$, the element $a^{n-1}$ is an absolute zero divisor of $J$.

Proof. For $i=n+1, n+2, \cdots, 2 n-1$, we have $Q\left(x^{i}\right)=Q\left(x^{i-n}\right) Q\left(x^{n}\right)=$ 0 , which proves (11).

Suppose now that the algebra $J$ satisfies the identities $x^{n+1}=x^{n+2}=$ $\cdots=x^{2 n-1}=0$. Since the ground field $F$ is infinite, the algebra $J$ satisfies also the following partial linearization of $x^{2 n-1}=0$ (see [Jac1, [ZSSS]):

$$
y Q\left(x^{n-1}\right)+x^{2(n-1)} \cdot y+\sum_{\substack{i+j=2(n-1) \\ 1 \leq i<j \leq 2 n-3}}\left\{x^{i}, y, x^{j}\right\}=0 .
$$

Hence, $y Q\left(x^{n-1}\right)=0$, which proves assertion of the lemma.

Let $J$ be a quadratic Jordan algebra, $a \in J$. Define a new structure of a quadratic Jordan algebra on $J$ via:

$$
x^{* 2}=a Q(x), y Q^{*}(x)=y Q(a) Q(x) .
$$

The new quadratic Jordan algebra is denoted as $J^{(a)}$ and is called a homotope of $J$ (see [Jac1, Jac4, McC2]).

For the quadratic Jordan algebra $\widetilde{J}=J \otimes_{F} E$ and an element $a \in \widetilde{J}$, consider the subspaces $K_{a}^{\prime}=\{x \in \widetilde{J} \mid x Q(a)=0\}$ and $K_{a}=\sum_{i}(J \otimes$ $\left.e_{i}+\widetilde{J} e_{i}\right) \cap K_{a}^{\prime}$. It is easy to see that the subspace $K_{a}$ is an ideal of the algebra $\widetilde{J}^{(a)}$.

Remark. If $p \neq 2$, then $K_{a}^{\prime}$ is also an ideal of $\widetilde{J}^{(a)}$.

Lemma 4.8. If $b \in J$ and $b+K_{a}$ is an absolute zero divisor of the algebra $J^{(a)} / K_{a}$, then $b Q(a)$ is an absolute zero divisor of the algebra $J$. 
Proof. We have $J Q(b Q(a))=J Q^{*}(b) Q(a) \subseteq K_{a} Q(a)=(0)$, which proves the lemma.

Lemma 4.9. Let a be an element of a quadratic Jordan algebra J. Let $f: \widetilde{J} \times \cdots \times \widetilde{J} \rightarrow \widetilde{J}$ be a homogeneous polynomial map, let $\widetilde{f}$ be its full linearization. Suppose that $\widetilde{f}\left(J \otimes e_{i}+\widetilde{J} e_{i}, \widetilde{J}, \cdots, \widetilde{J}\right) \subseteq J \otimes e_{i}+\widetilde{J} e_{i}$ for all $i$. If an arbitrary value of $f$ lies in $K_{a}^{\prime}$, then an arbitrary value of $f$ lies in $K_{a}$.

The proof is similar to the proof of Lemma 4.3 (2).

Let $f$ be an element of the free quadratic Jordan algebra $F J\langle X\rangle$, which is not an $S$-identity. Let $M=M(f)$ be the variety of quadratic Jordan algebras satisfying the identity $f=0$ (see [Jac1, Jac3, ZSSS]).

Definition. We say that a finite sequence of homogeneous elements $h_{1}, h_{2}, \cdots, h_{r} \in F J\langle X\rangle$ is an absolute zero divisor sequence for $M$ if for an arbitrary quadratic Jordan algebra $J \in M$

(i) every value of $h_{r}$ on $\widetilde{J}=J \otimes_{F} E$ is an absolute zero divisor of the algebra $\widetilde{J}$

(ii) if $h_{k}=h_{k+1}=\cdots=h_{r}=0$ identically hold on $\widetilde{J}, 2 \leq k \leq r$, then an arbitrary value of $h_{k-1}$ on $\widetilde{J}$ is an absolute zero divisor of $\widetilde{J}$.

Recall that in this section we always assume that $\operatorname{char} F=p>0$.

Proposition 4.10. For an arbitrary element $f \in F J\langle X\rangle$ that is not an $S$-identity the variety $M(f)$ has a finite absolute zero divisor sequence $h_{1}, h_{2}, \cdots, h_{r}$ with $h_{1}=x_{1} \in X$.

Proof. Let $F_{M}\langle X\rangle$ be the free algebra in the variety $M=M(f)$ on the set of free generators $X$. Since the factor algebra of $F_{M}\langle X\rangle$ modulo the $\mathrm{McCrimmon}$ radical can be approximated by prime nondegenerate algebras ([Zel3], The]), Lemma 4.6 implies that there exists $d \geq 1$ such that $y=\operatorname{Sym}_{d}\left(x_{1}, \cdots, x_{d}\right)$ lies in the McCrimmon radical of $F_{M}\langle X\rangle$. Consider the homotope algebra $F_{M}\langle X\rangle^{\left(x_{d+1}\right)}$ Since an absolute zero divisor of a Jordan algebra is an absolute zero divisor of every homotope, it follows that the McCrimmon radical of $F_{M}\langle X\rangle$ lies in the McCrimmon radical of $F_{M}\langle X\rangle^{\left(x_{d+1}\right)}$. In particular, the element $y$ lies in the McCrimmon radical of $F_{M}\langle X\rangle^{\left(x_{d+1}\right)}$ and therefore is nilpotent. 
Let $a^{\left(k, x_{d+1}\right)}$ denote the $k^{t h}$ power of an element $a$ in the homotope algebra $F_{M}\langle X\rangle^{\left(x_{d+1}\right)}$. There exists $m \geq 2$ such that $y^{\left(m-1, x_{d+1}\right)}=0$. Then $x_{d+1}^{(m, y)}=y^{\left(m-1, x_{d+1}\right)} Q\left(x_{d+1}\right)=0$. This implies that $x^{(m, y)}=0$ holds identically on $F_{M}\langle X\rangle$.

Then by Lemmas 4.7 and 4.8 , the sequence

$$
y, x_{d+1} Q(y), \cdots, x_{d+1}^{(m-1, y)} Q(y), x_{d+1}^{(2 m-1, y)} Q(y), \cdots, x_{d+1}^{(m+1, y)} Q(y)
$$

is an absolute zero divisor sequence in $M$.

Indeed, since the Jordan algebra $F_{M}\langle X\rangle^{(y)} / K_{y}$ satisfies the identity $x^{m}=0$, Lemma 4.7 (1) implies that the elements $x_{d+1}^{(2 m-1, y)}+K_{y}$, $\cdots, x_{d+1}^{(m+1, y)}+K_{y}$ are absolute zero divisors in $F_{M}\langle X\rangle^{(y)} / K_{y}$. By Lemma 4.8, the elements $x_{d+1}^{(2 m-1, y)} Q(y), \cdots, x_{d+1}^{(m+1, y)} Q(y)$ are absolute zero divisors of the algebra $F_{M}\langle X\rangle$.

If $J \in M$ and $x_{d+1}^{(2 m-1, y)} Q(y)=\cdots=x_{d+1}^{(m+1, y)} Q(y)=0$ hold identically on $\widetilde{J}$, then for arbitrary elements $a_{1}, \cdots, a_{d} \in \widetilde{J}$, $b=\operatorname{Sym}_{d}\left(a_{1}, \cdots, a_{d}\right), c \in \widetilde{J}$ the $i$-th power $c^{(i, b)}, m \leq i \leq 2 m-1$, lies in $K_{b}^{\prime}$. By Lemma 4.9, we have $c^{(i, b)} \in K_{b}$. In other words, the Jordan algebra $\widetilde{J}^{(b)} / K_{b}$ satisfies the identities $x^{m}=x^{m+1}=\cdots=x^{2 m-1}=0$. By Lemma 4.7 (2), for an arbitrary element $c \in \widetilde{J}$, the $(m-1)$-th power $c^{(m-1, b)}$ is an absolute zero divisor in $\widetilde{J}^{(b)} / K_{b}$. By Lemma 4.8, the element $c^{(m-1, b)} Q(b)$ is an absolute zero divisor of $\widetilde{J}$.

If $J \in M$ and $x_{d+1}^{(m-1, y)} Q(y)=x_{d+1}^{(2 m-1, y)} Q(y)=\cdots=x_{d+1}^{(m+1, y)} Q(y)=0$ holds identically on $\widetilde{J}$, then using Lemma 4.9 as above, we conclude that the algebra $\widetilde{J}^{(b)} / K_{b}$ satisfies the identities $x^{m-1}=\cdots=x^{2 m-1}=$ 0 .

Again, by Lemma 4.7 (2) and Lemma 4.8, every value of $x_{d+1}^{(m-2, y)} Q(y)$ (and so on) is an absolute zero divisor of $\widetilde{J}$.

If an algebra $J$ lies in $M$ and $y=\operatorname{Sym}_{d}\left(x_{1}, \cdots, x_{d}\right)=0$ holds identically on $J$ then the algebra $\widetilde{J}$ is nil of bounded index $\leq d$. Again by Lemma 4.7 we conclude that

$$
\begin{array}{r}
x_{1}, x_{1}^{2}, \cdots, x_{1}^{d-1}, x_{1}^{2 d-1}, \cdots, x_{1}^{d+1}, y, x_{d+1} Q(y), \cdots, \\
x_{d+1}^{(m-1, y)} Q(y), x_{d+1}^{(2 m-1, y)} Q(y), \cdots, x_{d+1}^{(m+1, y)} Q(y)
\end{array}
$$


is an absolute zero divisor sequence, which finishes the proof of the proposition.

Conjecture 4.11. If $J$ is a quadratic Jordan PI-algebra over a field of characteristic $p>0$ then the algebra $\widetilde{J}$ is nil of bounded index.

Now let's come back to the Lie algebra $L$ and the Jordan algebra $J_{a}=\widetilde{L}^{m} / K_{a}$.

Lemma 4.12. Let $b+K_{a}$ be a nonzero absolute zero divisor of the Jordan algebra $\widetilde{L}^{m} / K_{a}$. Then the element $b \mathrm{ad}^{[2]}(a)$ is a nonzero sandwich of the Lie algebra $\widetilde{L}^{m}$.

Proof. Let $b=\sum_{\pi} b_{\pi}$ be the standard decomposition. For an arbitrary element $c \in \widetilde{L}^{m}$ we have

$$
\left(c+K_{a}\right) Q\left(b+K_{a}\right)=\sum c \operatorname{ad}^{[2]}(a) \operatorname{ad}\left(b_{\pi_{1}}\right) \operatorname{ad}\left(b_{\pi_{2}}\right)+K_{a} .
$$

Hence by Lemma 4.2 ,

$$
\begin{gathered}
\sum c \operatorname{ad}^{[2]}(a) \operatorname{ad}\left(b_{\pi_{1}}\right) \operatorname{ad}\left(b_{\pi_{2}}\right) \operatorname{ad}^{[2]}(a) \\
=\sum c \operatorname{ad}\left(b_{\pi_{1}} \operatorname{ad}^{[2]}(a)\right) \operatorname{ad}\left(b_{\pi_{2}} \operatorname{ad}^{[2]}(a)\right)=0 .
\end{gathered}
$$

Let $\Omega=\left\{b_{\pi_{i}} \operatorname{ad}^{[2]}(a)\right\}$. We showed that $\widetilde{L}^{m} U_{2}(\Omega)=(0)$. By Lemma 3.13 the element $b \mathrm{ad}^{[2]}(a)$ is a sandwich of the Lie algebra $\widetilde{L}^{m}$.

Let $j\left(y_{1}, \cdots, y_{d}\right)$ be an arbitrary Jordan polynomial, i.e., an element of the free Jordan algebra. The polynomial $j$ defines a function $\widetilde{L}^{m} / K_{a} \times \cdots \times \widetilde{L}^{m} / K_{a} \rightarrow \widetilde{L}^{m} / K_{a}$ and, therefore, a function $\widetilde{L}^{m} \times \cdots \times \widetilde{L}^{m} \rightarrow \widetilde{L}^{m} / K_{a}$.

Lemma 4.13. Let $j\left(y_{1}, \cdots, y_{q}\right)$ be a multilinear Jordan polynomial. There exists a homogeneous divided polynomial $j^{\prime}\left(y_{1}, \cdots, y_{q}, x_{1}, \cdots, x_{r}\right)$ defined on $L^{m}$, such that the value $j\left(b_{1}, \cdots, b_{q}\right)$ in the Jordan algebra $\widetilde{L}^{m} / K_{a}$ is equal to $j^{\prime}\left(b_{1}, \cdots, b_{q}, a_{1}, \cdots, a_{r}\right)+K_{a}$. In particular,

$$
\begin{gathered}
j\left(b_{1}, \cdots, b_{q}\right) a d^{[2]} w\left(a_{1}, \cdots, a_{r}\right) \\
=j^{\prime}\left(b_{1}, \cdots, b_{q}, a_{1}, \cdots, a_{r}\right) a d{ }^{[2]} w\left(a_{1}, \cdots, a_{r}\right) .
\end{gathered}
$$


Proof. We will proceed by induction on the construction of the Jordan polynomial $j$. Let $j=\alpha j_{1}+\beta j_{2}$, where $\alpha, \beta \in F$ and $j_{1}, j_{2}$ are multilinear Jordan polynomials, such that the divided polynomials $j_{1}^{\prime}, j_{2}^{\prime}$ exist. Then we let $j^{\prime}=\alpha j_{1}^{\prime}+\beta j_{2}^{\prime}$. Suppose that $j=j_{1} \circ j_{2}$, where $j_{1}, j_{2}$ are multilinear Jordan polynomials on disjoint variables. let

We have $j\left(b_{1}, \cdots, b_{q}\right)=\left[a, j_{1}\left(b_{1}, \cdots, b_{q}\right), j_{2}\left(b_{1}, \cdots, b_{q}\right)\right]+K_{a}$ and we

$$
\begin{aligned}
& j^{\prime}\left(y_{1}, \cdots, y_{q}, x_{1}, \cdots, x_{r}\right)= \\
& {\left[w\left(x_{1}, \cdots, x_{r}\right), j_{1}^{\prime}\left(y_{1}, \cdots, y_{q}, x_{1}, \cdots, x_{r}\right), j_{2}^{\prime}\left(y_{1}, \cdots, y_{q}, x_{1}, \cdots, x_{r}\right)\right] .}
\end{aligned}
$$

Finally, let $j=\left\{j_{1}, j_{2}, j_{3}\right\}$, where $j_{1}, j_{2}, j_{3}$ are multilinear Jordan polynomials on disjoint variables. Arguing as above, we let

$$
j^{\prime}=\left[j_{2}^{\prime} \operatorname{ad}_{x_{1}}^{[2]} w\left(x_{1}, \cdots, x_{r}\right), j_{1}^{\prime}, j_{3}^{\prime}\right] .
$$

This completes the proof of the lemma.

Proposition 4.14. There exist integers $k \geq 1, t \geq 1$ and a homogeneous regular divided polynomial $v$ defined on $L^{k}$ such that every value of $v$ on $\widetilde{L}^{k}$ is a sum of $t$ sandwiches of the algebra $\widetilde{L}^{k}$.

Proof. Recall that there exists a homogeneous regular divided polynomial $w=w\left(x_{1}, \cdots, x_{r}\right)$ defined on $L^{m}, m \geq 1$, linear in $x_{r}$ and such that

(i) $\left[w\left(a_{1}, \cdots, a_{r-1}, a\right), w\left(a_{1}, \cdots, a_{r-1}, b\right)\right]=0$ for arbitrary elements $a, b, a_{1}, \cdots, a_{r-1} \in \widetilde{L}^{m}$

(ii) $\widetilde{L}^{m} \operatorname{ad}_{x_{r}}^{[t]}(w)=0$ holds identically on $\widetilde{L}^{m}$ for $t \geq 3$.

For an arbitrary $i \geq m$, arbitrary elements $a_{1}, \cdots, a_{r} \in \widetilde{L}^{i}$ consider $a=w\left(a_{1}, \cdots, a_{r}\right)$ and denote $\operatorname{ad}^{[2]}(a)=\operatorname{ad}_{x_{r}}^{[2]}\left(w\left(a_{1}, \cdots, a_{r}\right)\right)$.

Aruging as in the proof of Lemma 2.3, we conclude that there exists an element $f \in F J\langle X\rangle$ such that $f$ is not an $S$-identity and all quadratic Jordan algebras $\widetilde{L}^{i} / K_{a} ; i \geq m ; a_{1}, \cdots, a_{r} \in \widetilde{L}^{i}$ satisfy the identity $f=0$.

By Proposition 4.10 there exists an absolute zero divisor sequence $h_{1}=x_{1}, h_{2}, \cdots, h_{s}$ of the variety $M(f)$. 
If $J$ is an algebra from the variety $M(f)$ such that $J=\sum_{i} I_{i}, I_{i} \unlhd J$, $I_{i}^{2}=(0)$, then $J$ and $J \otimes_{F} E$ satisfy the same identities. Hence, every value of $h_{s}$ on $J$ is an absolute zero divisor of $J$ and if $h_{k}=\cdots=h_{s}=0$ identically hold on $J$, then every value of $h_{k-1}$ on $J$ is an absolute zero divisor.

Jordan algebras $\widetilde{L^{m}} / K_{a}$ that have been discussed above have this property. Indeed, $\widetilde{L^{m}} / K_{a}=\sum_{i} I_{i}$, where $I_{i}=L^{m} \otimes e_{i}+\widetilde{L^{m}} e_{i}+K_{a} / K_{a}$.

For an integer $i \geq m$ and elements $a_{1}, \cdots, a_{r} \in \widetilde{L}^{i}$ let $s\left(i, a_{1}, \cdots, a_{r}\right)$ be a maximal integer $j, 1 \leq j \leq s$, such that $h_{j}$ is not identically zero on $\widetilde{L}^{i} / K_{a}$. If $h_{1}=x_{1}$ is identically zero on $\widetilde{L}^{i} / K_{a}$, that is, $\widetilde{L}^{i}=K_{a}$, then we let $s\left(i, a_{1}, \cdots, a_{r}\right)=0$.

Let $s(i)=\max \left\{s\left(i, a_{1}, \cdots, a_{r}\right) \mid a_{1}, \cdots, a_{r} \in \widetilde{L}^{i}\right\}$. Clearly, $s(m) \geq$ $s(m+1) \geq \cdots$. Let this decreasing sequence stabilize at $t=s(k)=$ $s(k+1)=\cdots$.

If $t=0$ then $\widetilde{L^{k}}=K_{a}$, which means that $\widetilde{L^{k}} \operatorname{ad}_{x_{1}}^{[2]} w\left(a_{1}, \cdots, a_{r}\right)=(0)$ for arbitrary elements $a_{1}, \cdots, a_{r} \in \widetilde{L^{k}}$. By Lemma 3.13 every value of $w$ on $\widetilde{L^{k}}$ is a sandwich of the algebra $\widetilde{L^{k}}$. Therefore assume that $t \geq 1$.

Let us summarize the above. For arbitrary elements $a_{1}, \cdots, a_{r} \in$ $\widetilde{L^{k}}$ let $a=w\left(a_{1}, \cdots, a_{r}\right), \operatorname{ad}^{[2]}(a)=\operatorname{ad}_{x_{r}}^{[2]} w\left(a_{1}, \cdots, a_{r}\right), K_{a}=\widetilde{L^{k}} \cap$ ker $\operatorname{ad}^{[2]}(a)$. Every value of the Jordan polynomial $h_{t}$ on the Jordan algebra $\widetilde{L^{k}} / K_{a}$ is an absolute zero divisor. For every $k^{\prime} \geq k$ there exist elements $a_{1}, \cdots, a_{r} \in \widetilde{L^{k^{\prime}}}$ such that $h_{t}$ is not identically zero on $\widetilde{L^{k^{\prime}}} / K_{a}$. In particular, the Jordan polynomial $h_{t}$ is regular.

Suppose that $h_{t}=h_{t}\left(y_{1}, \cdots, y_{q}\right)$. Let $\mu$ be the total degree of the homogeneous Jordan polynomial $h_{t}$. The full linearization $\tilde{h}_{t}$ of the polynomial $h_{t}$ depends on $\mu$ variables. An arbitrary value of the polynomial $\widetilde{h_{t}}$ is a linear combination of $2^{\mu}=\ell$ values of the polynomial $h_{t}$. Let $\widetilde{h}_{t}^{\prime}\left(y_{1}, \cdots, y_{q}, x_{1}, \cdots, x_{r}\right)$ be the homogeneous divided polynomial of Lemma 4.13 defined on $\widetilde{L^{k}}$. Let $v\left(y_{1}, \cdots, y_{q}, x_{1}, \cdots, x_{r}\right)=$ $\widetilde{h_{t}^{\prime}}\left(y_{1}, \cdots, y_{q}, x_{1}, \cdots, x_{r}\right) \operatorname{ad}_{x_{r}}^{[2]} w\left(x_{1}, \cdots, x_{r}\right)$. 
For arbitrary elements $b_{1}, \cdots, b_{q}, a_{1}, \cdots, a_{r} \in \widetilde{L^{k}}$, we have

$$
\widetilde{h_{t}}\left(b_{1}, \cdots, b_{q}\right) \operatorname{ad}_{x_{r}}^{[2]} w\left(a_{1}, \cdots, a_{r}\right)=v\left(b_{1}, \cdots, b_{q}, a_{1}, \cdots, a_{r}\right) .
$$

We claim that the divided polynomial $v$ is regular. Indeed, it was shown above that for arbitrary $k^{\prime} \geq k$, there exist elements $a_{1}, \cdots, a_{r} \in$ $\widetilde{L^{k^{\prime}}}$ such that the Jordan polynomial $h_{t}$ is not identically zero on $\widetilde{L^{k^{\prime}}} / K_{a}$. Lemma 3.8 (2) was proved for arbitrary polynomial maps that include Jordan polynomials. Hence by Lemma 3.8 (2), the linear spans of the sets of values of the Jordan polynomials $h_{t}$ and $\widetilde{h}_{t}$ on the Jordan algebra $\widetilde{L^{k^{\prime}}} / K_{a}$ are equal. Hence $\widetilde{h_{t}}$ is not identically zero on $\widetilde{L^{k^{\prime}}} / K_{a}$. By Lemma 4.13, the homogeneous divided polynomial $v=\widetilde{h}_{t} \operatorname{ad}_{x_{r}}^{[2]}(w)$ is not identically zero on $\widetilde{L^{k^{\prime}}}$. This implies regularity of $v$.

By Lemma 4.12 every value of $v$ on $\widetilde{L^{k}}$ is a sum of $\ell=2^{\mu}$ sandwiches of the Lie algebra $\widetilde{L^{k}}$. This completes the proof of the proposition.

\section{SANDWICHeS IN $\widetilde{L}$}

Let $x \in \widetilde{L}, x=\sum_{\pi} x_{\pi}$ the standard decomposition. Suppose that

$$
\left[x_{\pi}, x_{\tau}\right]=0
$$

for arbitrary $\pi, \tau$. As above denote $\operatorname{ad}^{[k]}(x)=\sum \operatorname{ad}\left(x_{\pi_{1}}\right) \cdots \operatorname{ad}\left(x_{\pi_{k}}\right)$, where the sum runs over all $k$-element subsets $\left(\pi_{1}, \cdots, \pi_{k}\right)$. As we have already noticed in Lemma $\underline{3.7}(2), A(x)=\mathrm{Id}+\sum_{k=1}^{\infty} \mathrm{ad}^{[k]}(x)$ is an automorphism of the algebra $\widetilde{L}$.

Let elements $x_{1}, \cdots, x_{d} \in \widetilde{L}$ satisfy condition 5.1 , $A=A\left(x_{1}\right) \cdots A\left(x_{d}\right) \in$ Aut $\widetilde{L}$. The following lemma is straightforward. 
Lemma 5.1. For arbitrary elements $a_{1}, \cdots, a_{d} \in \widetilde{L}$ we have

$$
\begin{aligned}
& a_{1} A \otimes \cdots \otimes a_{d} A-\sum_{i=1}^{d} a_{1} A \otimes \cdots a_{i} \otimes \cdots \otimes a_{d} A+ \\
& \quad \sum_{1 \leq i \neq j \leq d} a_{1} A \otimes \cdots \otimes a_{i} \cdots \otimes a_{j} \cdots \otimes a_{d} A-\cdots \pm a_{1} \otimes \cdots \otimes a_{d}= \\
& \sum_{\sigma \in S_{d}}\left[a_{1}, x_{\sigma(1)}\right] \otimes \cdots \otimes\left[a_{d}, x_{\sigma(d)}\right] \\
& + \text { terms involving at least two elements from one of the sets }\left\{x_{i_{\pi}}\right\}_{\pi} .
\end{aligned}
$$

A. N. Grishkov Gri2 showed that in a Lie algebra over a field of zero characteristic a sandwich generates the locally nilpotent ideal. We will prove an analog of this result for the algebra $\widetilde{L}$. The proof essentially depends on the following result from [KZ]:

There exists a function $K Z: \mathbb{N} \rightarrow \mathbb{N}$ such that in an arbitrary Lie algebra if elements $a_{1}, \cdots, a_{n}$ are sandwiches then the subalgebra $\left\langle a_{1} \cdots, a_{n}\right\rangle$ is nilpotent of degree $\leq K Z(n)$.

Let $f(m, n)=K Z\left((n+1)^{m}\right)$.

Lemma 5.2. Let $I$ be an ideal of a Lie algebra $L, a \in \widetilde{I}$ a sandwich in $\widetilde{I}$. Let $S \subset \widetilde{L}$ be a finite set of $\leq n$ elements. Then the subalgebra of $\widetilde{L}$ generated by commutators $\left[a, b_{1} \cdots, b_{t}\right]$, where $b_{i} \in S, t \leq m$, is nilpotent of degree $\leq f(m, n)$.

Proof. Consider a commutator

$$
c=\left[\left[a, b_{11}, \cdots, b_{1 t_{1}}\right],\left[a, b_{21}, \cdots, b_{2 t_{2}}\right], \cdots,\left[a, b_{q 1}, \cdots, b_{q t_{q}}\right]\right],
$$

where $b_{i j} \in S, t_{i} \leq m, 1 \leq i \leq q, q=f(m, n)$. Our aim is to show that $c=0$.

Let an element $b \in S$ occur in the commutator $c|b|$ times. Clearly $\sum_{b \in S}|b|=t_{1}+\cdots+t_{q}$. Choose $|b|$ new elements $x_{b, 1}, \cdots, x_{b,|b|}$ in $\widetilde{L}$ and replace all $|b|$ occurrences of $b$ in $c$ by the symmetrized sum in $x_{b, 1}, \cdots, x_{b,|b|}$ :

$$
\cdots \underbrace{b \cdots b}_{|b|} \cdots \rightarrow \sum_{\sigma \in S_{|b|}} \cdots x_{b, \sigma(1)} \cdots x_{b, \sigma(|b|)} \cdots .
$$


We will get a new expression $c^{\prime}$ in $a, x_{b, j}$ 's, $b \in S, 1 \leq j \leq|b|$. Denote it $c^{\prime}=c^{\prime}\left(a, x_{b, 1}, \cdots, x_{b,|b|}\right)$. To show that $c=0$ it is sufficient to show that $c^{\prime}=0$. Indeed, let $b=b_{1}+\cdots+b_{k}$ be the standard decomposition of $b$. If $k<|b|$, then $c$ is a sum of expressions, each containing one of the elements $b_{1}, \cdots, b_{k}$ at least twice. If $k \geq|b|$, then $c=\sum c^{\prime}\left(a, b_{i_{1}}, \cdots, b_{i_{|b|} \mid}\right)$, where the sum runs over all $|b|$-element subsets of $\left\{b_{1}, \cdots, b_{k}\right\}$.

Let $x_{b j}=\sum_{\pi} x_{b j \pi}, a=\sum_{\pi} a_{\pi}$ be the standard decompositions, $x_{b j \pi}=x_{b j \pi}^{\prime} \otimes e_{\pi}, a_{\pi}=a_{\pi}^{\prime} \otimes e_{\pi}, x_{b j \pi}^{\prime} \in L, a_{\pi}^{\prime} \in I$.

Let $I(b, j)$ be the ideal of the Lie algebra $L$ generated by the subset $\left\{x_{b j \pi}\right\}_{\pi}$. Suppose at first that for arbitrary $b \in S, i \leq j \leq|b|$ we have

$$
[I(b, j), I(b, j)]=(0) .
$$

Then we can consider the automorphism

$$
A\left(x_{b, j}\right)=\mathrm{Id}+\sum_{k \geq 1} \operatorname{ad}^{[k]}\left(x_{b, j}\right), 1 \leq j \leq|b|
$$

and the automorphism $A(b)=A\left(x_{b, 1}\right) \cdots A\left(x_{b,|b|}\right)$. By Lemma 5.1 for arbitrary elements $a_{1}, \cdots, a_{|b|} \in \widetilde{L}$ we have

$$
\begin{aligned}
& a_{1} A(b) \otimes \cdots \otimes a_{|b|} A(b)-\sum a_{1} A(b) \otimes \cdots \otimes a_{i} \otimes \cdots \otimes a_{|b|} A(b)+ \\
& \sum a_{1} A(b) \otimes \cdots a_{i} \otimes \cdots \otimes a_{j} \otimes \cdots a_{|b|} A(b)-\cdots= \\
& \sum\left[a_{1}, x_{b, \sigma(1)}\right] \otimes \cdots \otimes\left[a_{|b|}, x_{b, \sigma(|b|)}\right] .
\end{aligned}
$$

Replacing each symmetric set $x_{b, 1}, \cdots, x_{b,|b|}$ in the element $c^{\prime}$ by expressions of the left hand side types we can represent $c^{\prime}$ as a linear combination of commutators

$$
\left[a \phi_{11} \cdots \phi_{1 m}, \cdots, a \phi_{q 1} \cdots \phi_{q m}\right]
$$

where each $\phi_{i j}$ is one of the automorphisms $A\left(b_{1}\right), \cdots, A\left(b_{n}\right), I d$. There are $\leq(n+1)^{m}$ elements $a \phi_{i 1}, \cdots \phi_{i m}$ and all of them are sandwiches in the algebra $\widetilde{I}$. By the choice of $q=K Z\left((n+1)^{m}\right)$ we conclude that $\left[a \phi_{11}, \cdots, \phi_{1 m}, \cdots, a \phi_{q 1} \cdots \phi_{q m}\right]=0$.

Now we will drop the assumption that

$$
[I(b, j), I(b, j)]=(0) .
$$

Let $\operatorname{Lie}\langle X\rangle$ be the free Lie algebra on the set of free generators $X=$ $\left\{\left\{x_{b, j, \pi}^{\prime}\right\}_{\pi},\left\{a_{\pi}\right\}_{\pi}\right\}$. Let $I(b, j)$ be the ideal of Lie $\langle X\rangle$ generated by the 
set $\left\{x_{b, j, \pi}^{\prime}\right\}_{\pi}$. Let $I=\sum_{b, j}[I(b, j), I(b, j)]$. Let $J$ be the ideal of $\operatorname{Lie}\langle X\rangle$ generated by all relations needed to make $a=\sum_{\pi} a_{\pi}^{\prime} e_{\pi}$ a sandwich in the ideal generated by $a$. So, $J$ is generated by

$$
\left[a_{\pi}^{\prime}, \rho, a_{\tau}^{\prime}\right]+\left[a_{\tau}^{\prime}, \rho, a_{\pi}^{\prime}\right],\left[a_{\pi}^{\prime}, \rho_{1}, \rho_{2}, a_{\tau}^{\prime}\right]+\left[a_{\tau}^{\prime}, \rho_{1}, \rho_{2}, a_{\pi}^{\prime}\right]
$$

where $\rho, \rho_{1}, \rho_{2}$ are arbitrary commutators in $X$ involving at least one element $a_{\mu}^{\prime}$.

Consider the Lie algebra $L=\operatorname{Lie}\langle X\rangle / I+J$. Since this algebra satisfies condition 5.2 the element $c^{\prime}$ computed in the algebra $\widetilde{L}$ lies in $(I+J) \otimes E$. The ideals $I$ and $J$ are graded with respect to each generator. The element $c^{\prime}$ has total degree one with respect to variables $\left\{x_{b, j, \pi}^{\prime}\right\}_{\pi}$ for each $b, j$. Since the ideal $I$ does not contain homogeneous elements having degree one with respect to all $\left\{x_{b, j, \pi}^{\prime}\right\}_{\pi}$ it follows that $c^{\prime} \in J \otimes E$. This finishes the proof of the lemma.

Now, let $A$ be an associative enveloping algebra of the algebra $L, L \subseteq$ $A^{(-)}, \widetilde{L}=L \otimes_{F} E, \widetilde{A}=A \otimes_{F} E$.

Lemma 5.3. Let $a \in \widetilde{L}$ be a sandwich. Then for an arbitrary element $b \in \widetilde{L}$ we have $[a, b]^{p}=0$.

Proof. N. Jacobson Jac2] noticed that

$$
\left\{x_{1}, \cdots, x_{p}\right\}=\sum_{\sigma \in S_{p}} x_{\sigma(1)} \cdots x_{\sigma(p)}=\sum_{\sigma \in S_{p-1}}\left[x_{p}, x_{\sigma(1)}, \cdots, x_{\sigma(p-1)}\right] .
$$

Let $b=\sum_{\pi} b_{\pi}$ be the standard decomposition of the element $b$. Then $[a, b]=\sum_{\pi}^{\pi}\left[a, b_{\pi}\right]$ and $[a, b]^{p}=\sum\left\{\left[a, b_{\pi_{1}}\right], \cdots,\left[a, b_{\pi_{p}}\right]\right\}$. Each summand on the right hand side is equal to zero since $\left[\left[a, b_{\pi_{i}}\right],\left[a, b_{\pi_{j}}\right]\right]=0$, which proves the lemma.

Lemma 5.4. Let $I$ be an ideal of the Lie algebra L. Let $a \in \widetilde{I}$ be a sandwich in $\widetilde{I}$ such that $a^{p}=0$ in the algebra $\widetilde{A}$. Let $S \subset \widetilde{L}$ be a finite set of $\leq n$ elements. Then the associative subalgebra of $\widetilde{A}$ generated by commutators $\left[a, b_{1}, \cdots, b_{t}\right]$, where $b_{i} \in S, t \leq m$, is nilpotent of degree $\leq p^{(n+1)^{m f(m, n)}}$. 
Proof. Denote $q=p^{(n+1)^{m f(m, n)}}$. We need to show that an arbitrary product

$$
\left[a, b_{11}, \cdots, b_{1 t_{1}}\right] \cdots\left[a, b_{q 1}, \cdots, b_{q t_{q}}\right],
$$

where $b_{i j} \in B, t_{i} \leq m, 1 \leq i \leq q$, is equal to 0 .

Arguing as in the proof of Lemma 5.2 we can reduce the problem to showing that an arbitrary product $\left(a \phi_{11} \cdots \phi_{1 m}\right) \cdots\left(a \phi_{q 1} \cdots \phi_{q m}\right)=0$, where $\phi_{i j} \in$ Aut $\widetilde{A}, \phi_{i j}(\widetilde{L})=\widetilde{L}, \phi_{i j}(\widetilde{I})=\widetilde{I}, \#\left\{\phi_{i j} \mid 1 \leq i \leq q, 1 \leq j \leq\right.$ $m\} \leq n+1$.

Let $Y=\left\{a \phi_{i 1} \cdots \phi_{i m}, 1 \leq i \leq q\right\},|Y| \leq(n+1)^{m}$. An arbitrary element $y$ from $Y$ is a sandwich in $\widetilde{I}$ and $y^{p}=0$ in $\widetilde{A}$.

Let $L_{1}$ be the Lie algebra generated by $Y$. By Lemma $5.2 L_{1}^{f(m, n)}=$ (0). Let $\rho_{1}, \cdots, \rho_{r}$ be left normed commutators in $Y$ that form a basis of $L_{1}, r \leq|Y|^{f(m, n)} \leq(n+1)^{m f(m, n)}$.

By Lemma 5.3 for each commutator $\rho_{i}$ we have $\rho_{i}^{p}=0$. Now the Poincare-Birkhoff-Witt theorem implies the assertion of the lemma.

\section{Proof of Theorem 1.1}

Let a Lie algebra $L$ over a field $F$ of characteristic $p>0$ and its associative enveloping algebra $A, L \subseteq A^{(-)}, A=\langle L\rangle$, satisfy the conditions outlined at the beginning of $\S 3$ :

1) $L$ is a graded Lie algebra generated by elements $x_{1}, \cdots, x_{m}$ of degree 1; every element from the Lie set $S=S\left\langle x_{1}, \cdots, x_{m}\right\rangle$ is ad-nilpotent;

2) $L$ satisfies a polynomial identity;

3 ) the grading of $L$ extends to $A$; both algebras $L$ and $A$ are graded just infinite.

Recall that an element $g\left(x_{1}, \cdots, x_{r}\right)$ of the free associative algebra is called a weak identity of the pair $(L, A)$ if $g\left(a_{1}, \cdots, a_{r}\right)=0$ for arbitrary elements $a_{1}, \cdots, a_{r} \in L$. In particular, every Lie identity of the algebra $L$ can be viewed as a weak identity of the pair $(L, A)$.

Let $k$ be a minimal degree of a nonzero weak identity satisfied by $(L, A)$. Without loss of generality we can assume that $(L, A)$ satisfies 
a weak identity

$$
h\left(x_{1}, \cdots, x_{k}\right)=x_{1} \cdots x_{k}+\sum_{1 \neq \sigma \in S_{k}} \alpha_{\sigma} x_{\sigma(1)} \cdots x_{\sigma(k)} .
$$

We remark that the pair $(\widetilde{L}, \widetilde{A})$ satisfies this weak identity as well.

Let $h\left(x_{1}, \cdots, x_{k}\right)=\sum_{i=1}^{k} h_{i}\left(x_{1}, \cdots, \hat{x}_{i}, \cdots, x_{k}\right) x_{i}$. The ideal $M$ of the algebra $A$ generated by all values of $h_{k}\left(a_{1}, \cdots, a_{k-1}\right), a_{i} \in L$ is graded nonzero and therefore has finite codimension in $A$. Hence there exists $d \geq 1$ such that $A^{d} \subseteq M$. Denote $\widehat{A}=A+F 1$,

Lemma 6.1. For an arbitrary element $a \in \widetilde{L}$ we have

$$
A^{d} a \subseteq \sum x_{i_{1}} \cdots x_{i_{t}} a \widehat{A}, t \leq d-1 .
$$

Proof. For an arbitrary product of length $d$ we have

$$
x_{i_{1}} \cdots x_{i_{d}}=\sum_{j} \alpha_{j} v_{j}^{\prime} h_{k}\left(\rho_{j 1}, \cdots, \rho_{j, k-1}\right) v_{j}^{\prime \prime},
$$

where $\alpha_{j} \in F ; v_{j}^{\prime}, v_{j}^{\prime \prime}$, and $\rho_{j 1}, \cdots, \rho_{j, k-1}$ are monomials and commutators in generators $x_{1}, \cdots, x_{m}$ of total length $d$.

Let $v_{j}^{\prime \prime}=x_{\mu_{1}} \cdots x_{\mu_{r}}$. Then

$$
v_{j}^{\prime \prime} a=a^{\prime}+\sum_{t} \pm w_{j t}^{\prime} a w_{j t}^{\prime \prime},
$$

where $a^{\prime}=\left[x_{\mu_{1}},\left[x_{\mu_{2}},\left[\cdots,\left[x_{\mu_{r}}, a\right] \cdots\right] ; w_{j t}^{\prime}, w_{j t}^{\prime \prime}\right.\right.$ are products in generators of total degree equal to the degree of $v_{j}^{\prime \prime}$ and the products $w_{j t}^{\prime \prime}$ are not empty. Hence,

$$
\begin{array}{r}
x_{i_{1}} \cdots x_{i_{d}} a=\sum_{j} \alpha_{j} v_{j}^{\prime} h_{k}\left(\rho_{j, 1}, \cdots, \rho_{j, k-1}\right) a^{\prime}+ \\
\sum_{j, t} \alpha_{j} v_{j}^{\prime} h_{k}\left(\rho_{j, 1}, \cdots, \rho_{j, k-1}\right) w_{j t}^{\prime} a w_{j t}^{\prime \prime} .
\end{array}
$$

Furthermore,

$$
\begin{aligned}
& h_{k}\left(\rho_{j 1}, \cdots, \rho_{j, k-1}\right) a^{\prime}=h\left(\rho_{j 1}, \cdots, \rho_{j, k-1}, a^{\prime}\right)- \\
& \quad \sum_{i=1}^{k-1} h_{i}\left(\rho_{j 1}, \cdots, a^{\prime}, \cdots, \rho_{j, k-1}\right) \rho_{j i}=-\sum_{i=1}^{k-1} h_{i}\left(\rho_{j 1}, \cdots, a^{\prime}, \cdots \rho_{j, k-1}\right) \rho_{j i} .
\end{aligned}
$$


We proved that $x_{i_{1}} \cdots x_{i_{d}} a$ is a linear combination of elements $w^{\prime} a w^{\prime \prime}$, where $w^{\prime}, w^{\prime \prime}$ are products in $x_{1}, \cdots, x_{m}$, with the length of $w^{\prime}$ less than $d$.

Consider the function $g(m, n)=p^{(n+1)^{m f(m, n)}}$.

Lemma 6.2. Let $I$ be an ideal of the algebra L. Let a be a sandwich in $\widetilde{I}$ and $a^{p}=0$ in $\widetilde{A}$. Let $a^{\prime}=\left[a_{1}, \cdots, a_{d}\right]$, where $a_{i}=\left[a, u_{i 1}, \cdots, u_{i t_{i}}\right], u_{i j} \in$ $\widetilde{L}, t_{i} \geq 0, i=1, \cdots, d$. Let $\left.G=g\left(\max _{i}\left\{t_{i}+d-1\right\}, t_{1}+\cdots+t_{d}+d^{d+1}\right\}\right)$. Then $\left(A a^{\prime}\right)^{G}=(0)$. In particular, an arbitary element from $\operatorname{Id}_{\widetilde{L}}\left(a^{\prime}\right)^{d}$ generates a nilpotent ideal in $\widetilde{A}$.

Proof. Suppose that $a^{\prime} v_{1} a^{\prime} \cdots v_{G-1} a^{\prime} \neq 0$, where $v_{1}, \cdots, v_{G-1}$ are products in generators $x_{1}, \cdots, x_{m}$ of lengths $l\left(v_{1}\right), \cdots, l\left(v_{G-1}\right)$ respectively. Without loss of generality we will assume that the vector of lengths $\left(l\left(v_{1}\right), \cdots, l\left(v_{G-1}\right)\right)$ is lexicographically minimal. By Lemma 6.1 this implies that $l\left(v_{i}\right) \leq d-1,1 \leq i \leq G-1$.

If $v=x_{j_{1}} \cdots x_{j_{r}}$ then we denote

$$
\left[v a^{\prime}\right]=\left[x_{j_{1}},\left[x_{j_{2}},\left[\cdots\left[x_{j_{r}}, a^{\prime}\right] \cdots\right]=(-1)^{r}\left[a^{\prime}, x_{j_{r}}, x_{j_{r-1}}, \cdots, x_{j_{1}}\right] .\right.\right.
$$

Again by lexicographical minimality we have

$$
a^{\prime} v_{1} a^{\prime} \cdots v_{G-1} a^{\prime}=a^{\prime}\left[v_{1} a^{\prime}\right] \cdots\left[v_{G-1} a^{\prime}\right] .
$$

Lemma 5.4 is not applicable to the sandwich $a^{\prime}$ and elements $x_{1}, \cdots, x_{m}$ because $x_{1}, \cdots, x_{m}$ do not lie in $\widetilde{L}$. However, for an arbitrary word $v=x_{j_{1}} \cdots x_{j_{r}}, r<d$, we have

$$
\left[v a^{\prime}\right]=\sum_{i}\left[\left[v_{i 1} a_{1}\right], \cdots,\left[v_{i d} a_{d}\right]\right]
$$

where $v_{i 1}, \cdots, v_{i d}$ are words in $x_{1}, \cdots, x_{m}$ of total length $r<d$. Hence, at least one of these words is empty. Now we can apply Lemma 5.4 to the sandwich $a$ and the set $S=\left\{\left[a, u_{i 1}, \cdots, u_{i t_{i}}, x_{j 1}, \cdots, x_{j r}\right] \mid 1 \leq\right.$ $\left.i \leq d, 1 \leq j_{1}, \cdots, j_{r} \leq m, 0 \leq r<d\right\} \subset \widetilde{L}$. By Lemma 5.4 and the choice of the number $G$, we have $a^{\prime}\left[v_{1} a^{\prime}\right] \cdots\left[v_{G-1} a^{\prime}\right]=0$, which proves the lemma.

Now our aim is the following: 
Proposition 6.3. There exist integers $N \geq 1, s \geq 1$ and a regular divided polynomial $v$ defined on $L^{s}$ such that every value of $v$ on $\widetilde{L}^{s}$ generates a nilpotent ideal in $\widetilde{A}$ of degree $\leq N$.

Suppose that the algebra $L$ satisfies an identity $f\left(x_{0}, x_{1}, \cdots, x_{n-1}\right)=$ $\left[x_{0}, x_{1}, \cdots, x_{n-1}\right]+\sum_{1 \neq \sigma \in S_{n-1}} \alpha_{\sigma}\left[x_{0}, x_{\sigma(1)}, \cdots, x_{\sigma(n-1)}\right]$, where $\alpha_{\sigma} \in F$ and $n$ is the minimal degree of an identity satisfied by $L$.

Consider the following element of degree $n-1$ :

$$
\begin{aligned}
& f_{1}\left(x_{0}, x_{2}, \cdots, x_{n-1}\right)=\left[x_{0}, x_{2}, \cdots, x_{n-1}\right]+ \\
& \sum_{\substack{1 \neq \sigma \in S_{n-1} \\
\sigma(1)=1}} \alpha_{\sigma}\left[x_{0}, x_{\sigma(2)}, \cdots, x_{\sigma(n-1)}\right]=x_{0} H\left(\operatorname{ad}\left(x_{2}\right), \cdots, \operatorname{ad}\left(x_{n-1}\right)\right),
\end{aligned}
$$

where $H\left(y_{2}, \cdots, y_{n-1}\right)=y_{2} \cdots y_{n-1}+\sum_{\substack{1 \neq \sigma \in S_{n-1} \\ \sigma(1)=1}} \alpha_{\sigma} y_{\sigma(2)} \cdots y_{\sigma(n-1)}$.

Lemma 6.4. Let $w\left(x_{1}, \cdots, x_{r}\right)$ be a regular divided polynomial defined on $L^{s}$. Then the divided polynomial

$$
w^{\prime}\left(x_{1}, \cdots, x_{r}, y_{2}, \cdots, y_{n-1}\right)=f_{1}\left(w\left(x_{1}, \cdots, x_{r}\right), y_{2}, \cdots, y_{n-1}\right)
$$

is also regular.

Proof. Choose $t \geq s$ and elements $a_{1}, \cdots, a_{r} \in \widetilde{L}^{t}$ such that $a^{\prime}=$ $w\left(a_{1}, \cdots, a_{r}\right) \neq 0$. Suppose that for arbitrary elements $b_{2}, \cdots, b_{n-1} \in$ $\widetilde{L}^{t}$ we have $f_{1}\left(a^{\prime}, b_{2}, \cdots, b_{n-1}\right)=0$. Let $a^{\prime}=\sum_{\pi} a_{\pi}^{\prime}$ be the standard decomposition, $a_{\pi}^{\prime}=\overline{a_{\pi}^{\prime}} \otimes e_{\pi}, \overline{a_{\pi}^{\prime}} \in L^{t}$. Then the assumption above means that for an arbitrary $\pi$ we have $f_{1}\left(\bar{a}_{\pi}^{\prime}, L^{t}, \cdots, L^{t}\right)=(0)$. Choose $\pi$ such that $\overline{a_{\pi}^{\prime}} \neq 0$. Let $R=\langle\operatorname{Id}, \operatorname{ad}(x), x \in L\rangle \subseteq \operatorname{End}_{F}(L)$ be the multiplication algebra of the algebra $L$. Consider the ideal $\operatorname{id}_{L}\left(\overline{a_{\pi}^{\prime}}\right)=$ $\overline{a_{\pi}^{\prime}} R$ generated by the element $\overline{a_{\pi}^{\prime}}$ in $L$.

For an arbitrary element $x \in L$ we have

$$
\left[\operatorname{ad}(x), H\left(\operatorname{ad}\left(L^{t}\right), \cdots, \operatorname{ad}\left(L^{t}\right)\right)\right] \subseteq H\left(\operatorname{ad}\left(L^{t}\right), \cdots, \operatorname{ad}\left(L^{t}\right)\right) .
$$

Hence,

$$
R H\left(\operatorname{ad}\left(L^{t}\right), \cdots, \operatorname{ad}\left(L^{t}\right)\right) \subseteq H\left(\operatorname{ad}\left(L^{t}\right), \cdots, \operatorname{ad}\left(L^{t}\right)\right) R .
$$


Now for an arbitrary operator $P \in R$ we have

$$
\begin{aligned}
& f_{1}\left(\overline{a_{\pi}^{\prime}} P, L^{t}, \cdots, L^{t}\right)=\overline{a_{\pi}^{\prime}} P H\left(L^{t}, \cdots, L^{t}\right) \\
& \quad \subseteq \bar{a}_{\pi}^{\prime} H\left(L^{t}, \cdots, L^{t}\right) R=f_{1}\left(\overline{a_{\pi}^{\prime}}, L^{t}, \cdots, L^{t}\right) R=(0) .
\end{aligned}
$$

Since the algebra $L$ is graded just infinite it follows that $\operatorname{id}_{L}\left(\overline{a_{\pi}^{\prime}}\right) \supseteq L^{k}$ for sufficiently large $k \geq t$. We proved that $f_{1}\left(L^{k}, \cdots, L^{k}\right)=(0)$.

The algebra $L^{k}$ is finitely generated by Lemma 2.4. By the induction assumption on the degree of the identity $f$ we conclude that the Lie algebra $L^{k}$ is finite dimensional. Therefore the algebra $L$ is finite dimensional as well which contradicts our assumption that the algebra $L$ is graded just infinite, proving the lemma.

Corollary 6.5. Let $q \geq 1$. Choose $r+q(n-2)$ distinct variables $x_{1}, \cdots, x_{r}, y_{i j}, 1 \leq i \leq q, 2 \leq j \leq n-1$. Then the divided polynomial

$$
\begin{array}{r}
w_{q}=w\left(x_{1}, \cdots, x_{r}\right) H\left(\operatorname{ad}\left(y_{11}\right), \cdots, \operatorname{ad}\left(y_{1, n-1}\right)\right) \cdots \\
H\left(\operatorname{ad}\left(y_{q 1}, \cdots, \operatorname{ad}\left(y_{q, n-1}\right)\right)\right.
\end{array}
$$

is regular.

Lemma 6.6. For arbitrary elements $a, b_{2}, \cdots, b_{n-1}, c \in \widetilde{L}$ we have

$$
\left[f_{1}\left(a, b_{2}, \cdots, b_{n-1}\right), c\right] \in \sum F\left[a, b_{i_{2}}, \cdots,\left[b_{i_{k}}, c\right], \cdots, b_{i_{n-1}}\right]
$$

where $i_{2}, i_{3}, \cdots, i_{n-1}$ is a permutation of $2, \cdots, n-1 ; 2 \leq k \leq n-1$.

Proof. We have

$$
\begin{array}{r}
a H\left(\operatorname{ad}\left(b_{2}\right), \cdots, \operatorname{ad}\left(b_{n-1}\right) \operatorname{ad}(c)=a \operatorname{ad}(c) H\left(\operatorname{ad}\left(b_{2}\right), \cdots, \operatorname{ad}\left(b_{n-1}\right)\right)+\right. \\
\sum a H\left(\operatorname{ad}\left(b_{2}\right), \cdots, \operatorname{ad}\left(\left[b_{k}, c\right]\right), \cdots, \operatorname{ad}\left(b_{n-1}\right)\right) .
\end{array}
$$

Let us represent the polynomial $f$ of minimal degree as $f\left(x_{0}, x_{1}, \cdots, x_{n-1}\right)=\sum_{i=1}^{n-1} x_{0} \operatorname{ad}\left(x_{i}\right) H_{i}\left(\operatorname{ad}\left(x_{1}\right), \cdots, \widehat{\operatorname{ad}\left(x_{i}\right)}, \cdots, \operatorname{ad}\left(x_{n-1}\right)\right)$,

where $H_{1}=H$. Then

$$
\begin{aligned}
& a \operatorname{ad}(c) H\left(\operatorname{ad}\left(b_{2}\right), \cdots, \operatorname{ad}\left(b_{n-1}\right)\right)=-c \operatorname{ad}(a) H\left(\operatorname{ad}\left(b_{2}\right), \cdots, \operatorname{ad}\left(b_{n-1}\right)\right) \\
& =\sum_{i=2}^{n-1}\left[c, b_{i}\right] H_{i}\left(\operatorname{ad}(a), \operatorname{ad}\left(b_{2}\right), \cdots, \widehat{\operatorname{ad}\left(b_{i}\right)}, \cdots, \operatorname{ad}\left(b_{n-1}\right)\right) \\
& \in \sum F\left[a, b_{i_{2}}, \cdots,\left[b_{i_{k}}, c\right], \cdots, b_{i_{n-1}}\right] .
\end{aligned}
$$


Lemma 6.7. Let $\nu \geq 1$. Suppose that a divided polynomial $w\left(x_{1}, \cdots, x_{r}\right)$ is defined on $L^{s}$ and for all $t \leq q(n-2)+\nu$ the divided polynomial $\left[w\left(x_{1}, \cdots, x_{r}\right), y_{1}, \cdots, y_{t}, w\left(x_{1}, \cdots, x_{r}\right)\right]$ is identically zero on $\widetilde{L}^{s}$. Then for arbitrary elements $a_{k}, b_{i j} \in \widetilde{L}^{s}$ we have

$$
\begin{array}{r}
{\left[w_{q}\left(a_{k}, b_{i j}, 1 \leq k \leq r, 1 \leq i \leq q, 2 \leq j \leq n-1\right),\right.} \\
\underbrace{L, L, \cdots, L}_{\mu}, \underbrace{L^{s}, \cdots, L^{s}}_{\nu}, w\left(a_{1}, \cdots, a_{r}\right)]=(0)
\end{array}
$$

for $\mu \leq q$

Proof. Applying Lemma 6.6 $q$ times we get

$$
[w_{q}\left(a_{k}, b_{i j}\right), \underbrace{L, \cdots, L}_{\mu}] \subseteq[w\left(a_{1}, \cdots, a_{r}\right), \underbrace{\widetilde{L}^{s}, \cdots, \widetilde{L}^{s}}_{q(n-2)}]
$$

which implies the assertion of the lemma.

Lemma 6.8. Let $a \in \widetilde{L}$ and $[a, \underbrace{\widetilde{L}, \cdots, \widetilde{L}}_{\mu}, a]=(0)$ for $\mu \leq 2 d$. Then for an arbitrary element $b \in \widetilde{L}$ the commutator $[a, b]$ generates a nilpotent ideal in $\widetilde{A}$ of degree $\leq m^{d}(p-1)+1$.

Proof. Recall that the algebra $L$ is generated by $m$ elements $x_{1}, \cdots, x_{m}$. Suppose that $[a, b] v_{1}[a, b] \cdots v_{N-1}[a, b] \neq 0$, where $v_{i}$ are products of the generators and the vector of lengths $\left(l\left(v_{1}\right), \cdots, l\left(v_{N-1}\right)\right.$ is lexicographically minimal among all vectors with this property. Then by Lemma 6.1 $l\left(v_{i}\right)<d$ for $i=1, \cdots, N-1$.

As above, for a product $v=x_{i_{1}}, \cdots, x_{i_{k}}$ we denote

$$
[v[a, b]]=\left[x _ { i _ { 1 } } \left[x_{i_{2}},\left[\cdots\left[x_{i_{k}},[a, b]\right] \cdots\right] .\right.\right.
$$

We have

$$
[a, b] v_{1} \cdots v_{N-1}[a, b]=[a, b]\left[v_{1},[a, b]\right] \cdots\left[v_{N-1}[a, b]\right] .
$$

By our assumption the commutators $[a, b],\left[v_{i}[a, b]\right], 1 \leq i \leq N-1$, commute. There are $<m^{d}$ such commutators. Hence at least one commutator $\left[v_{i}[a, b]\right]$ occurs $\geq p$ times. If $b=\sum_{\pi} b_{\pi}$ is the standard decomposition then $\left[v_{i}[a, b]\right]^{p}$ is a sum of expressions

$$
\begin{aligned}
& \left\{\left[v_{i}\left[a, b_{\pi_{0}}\right]\right], \cdots,\left[v_{i}\left[a, b_{\pi_{p-1}}\right]\right]\right\}= \\
& \sum_{\sigma \in S_{p-1}}\left[\left[v_{i},\left[a, b_{\pi_{0}}\right]\right],\left[v_{i}\left[a, b_{\pi_{\sigma(1)}}\right]\right], \cdots,\left[v_{i}\left[a, b_{\pi_{\sigma(p-1)}}\right]\right]\right]=0 .
\end{aligned}
$$


Hence $\left[v_{i}[a, b]\right]^{p}=0$, which finishes the proof of the lemma.

Lemma 6.9. For an arbitrary sandwich of the algebra $\widetilde{L}^{s}$ we have $a^{p}=0$ in $\widetilde{A}$.

Proof. Recall that the algebra $A$ is a homomorphic image of the subalgebra $\langle\operatorname{ad}(x), x \in L\rangle \subseteq \operatorname{End}_{F}(L)$.

Hence it is sufficient to show that $\operatorname{ad}(a)^{p}=0$ in $\widetilde{L}$. If $p \geq 3$ then $\widetilde{L} \operatorname{ad}(a)^{p} \subseteq\left[\widetilde{L}^{s}, a, a\right]=(0)$. Let $p=2$. We have $\left[\widetilde{L}^{s}, a, a\right]=(0)$. Since the mapping $\widetilde{L} \rightarrow \widetilde{L}, x \rightarrow[x, a, a]$ is a derivation of $\widetilde{L}$ it follows that $[\widetilde{L}, a, a]$ lies in the centralizer of $\widetilde{L}^{s}$. In a graded just infinite algebra $L$ the centralizer of $L^{s}$ is zero. Hence $[\widetilde{L}, a, a]=(0)$ and again $\operatorname{ad}(a)^{2}=0$, which finishes the proof of the lemma.

Proof of Proposition 6.3. We will start with the regular divided polynomial $v\left(x_{1}, \cdots, x_{r}\right)$ of Proposition 4.14 defined on $L^{s}$. Every value of $v$ on $\widetilde{L}^{s}$ is a sum of $t$ sandwiches of the algebra $\widetilde{L}^{s}$.

We will construct a sequence of finite sets $M_{i}$ of divided polynomials defined on $L^{s}, i \geq 0$.

Let $M_{0}=\{v\}, M_{i+1}=\left\{\left[w, y_{1}, \cdots, y_{\mu}, w\right] \mid w \in M_{i}, \mu \leq 4 d(n-\right.$ $2) ; y_{1}, \cdots, y_{\mu}$ are variables not involved in $\left.w\right\}$.

Let $2^{i} \geq(d-1) t+1, w \in M_{i}$. Let $b$ be a value of the divided polynomial $v$ on $\widetilde{L}^{s}$. By Proposition 4.14, $b=b_{1}+\cdots+b_{t}$, where for $1 \leq j \leq t$, each element $b_{j}$ is a sandwich of the algebra $\widetilde{L}^{s}$. Since $2^{i} \geq(d-1) t+1$, it follows that the value $c$ of the divided polynomial $w$ that is obtained by iterating the value $b$ of $v$ lies in $\sum_{j=1}^{t} \operatorname{id}_{\widetilde{L}}\left(b_{j}\right)^{d}$. By Lemma 6.2 there exists an integer $G \geq 1$ such that every value of the divided polynomial $w$ on $\widetilde{L}^{s}$ generates a nilpotent ideal in $\widetilde{A}$ of degree $\leq G$.

If at least one divided polynomial in $M_{i}$ is regular, then we are done.

If none of the divided polynomials in $M_{i}$ is regular and $i$ is the minimal integer with this property then there exists a regular divided polynomial $w\left(x_{1}, \cdots, x_{r}\right)$ defined on $\widetilde{L}^{s}$ and an integer $t \geq s$ such that 
all the divided polynomials

$$
\left[w\left(x_{1}, \cdots, x_{r}\right), y_{1}, \cdots, y_{\mu}, w\left(x_{1}, \cdots, x_{r}\right)\right], 1 \leq \mu \leq 4 d(n-2),
$$

are identically zero on $\widetilde{L}^{t}$.

Consider the regular divided polynomial $w_{2 d}\left(x_{1}, \cdots, x_{r}, y_{i j}, 1 \leq i \leq\right.$ $2 d, 2 \leq j \leq n-1)$. By Lemma 6.7 we have

$$
[w_{2 d}\left(a_{1}, \cdots, a_{r}, b_{i j}\right), \underbrace{L, L, \cdots, L}_{\mu}, \underbrace{L^{t}, \cdots, L^{t}}_{\nu}, w\left(a_{1}, \cdots, a_{r}\right)]=(0),
$$

$\mu \leq 2 d, \nu \leq 2 d(n-2)$ for arbitrary elements $a_{1}, \cdots, a_{r}, b_{i j} \in \widetilde{L}^{t}$. We have $w_{2 d}\left(a_{1}, \cdots, a_{r}, b_{i j}\right) \in \sum_{\nu=1}^{2 d(n-2)}[w\left(a_{1}, \cdots, a_{r}\right), \underbrace{L^{t}, \cdots, L^{t}}_{\nu}]$. Therefore

$$
[w_{2 d}\left(a_{1}, \cdots, a_{r}, b_{i j}\right), \underbrace{L, L, \cdots, L}_{\mu}, w_{2 d}\left(a_{1}, \cdots, a_{r}, b_{i j}\right)]=(0)
$$

for $\mu \leq 2 d$.

The divided polynomial

$$
w_{2 d}^{\prime}\left(x_{0}, x_{1}, \cdots, x_{r}, y_{i j}\right)=\left[w_{2 d}\left(x_{1}, \cdots, x_{r}, y_{i j}\right), x_{0}\right]
$$

is regular. Indeed, regularity of $w_{2 d}$ has been established in Corollary 6.5. If the divided polynomial $w_{2 d}^{\prime}$ vanishes on some power of $L$, then some power of $L$ has a nonzero centralizer. Since the algebra $L$ is graded just infinite, it follows that this centralizer is of finite codimension. Hence the algebra $L$ is solvable and therefore finite dimensional, a contradiction. By Lemma 6.8 an arbitrary value of $w_{2 d}^{\prime}$ on $\widetilde{L}^{t}$ generates a nilpotent ideal in $\widetilde{A}$ of degree $\leq m^{d}(p-1)+1$. This finishes the proof of Proposition 6.3.

By Proposition 6.3 there exist integers $s \geq 1, N_{1} \geq 1$ and a regular divided polynomial $w$ defined on $L^{s}$ such that every value of $w$ on $\widetilde{L}^{s}$ generates a nilpotent ideal in $\widetilde{A}$ of degree $\leq N_{1}$. Let $f\left(x_{1}, x_{2}, \cdots, x_{r}\right) \in$ $\widetilde{L} * \operatorname{Lie}\langle X\rangle$ be the linearization of the divided polynomial $w$. If $l$ is the degree of $w$ then every value of $f$ in $\widetilde{L}^{s}$ is a linear combination of $2^{l}$ values of $w$. Hence for arbitrary $a_{1}, a_{2}, \cdots a_{r} \in \widetilde{L}^{s}$ the element $f\left(a_{1}, \cdots, a_{r}\right)$ generates a nilpotent ideal in $\widetilde{A}$ of degree $\leq N_{2}=2^{l}\left(N_{1}-\right.$ 1) +1 . 
Choose variables $x_{i j} \in X, 1 \leq i \leq r, 1 \leq j \leq N_{2}$. The pair $(L, A)$ satisfies the system of weak identities

$$
\begin{aligned}
& F_{N_{2}}=\left\{F_{N_{2}}\left(x_{i j}, y_{k}\right)=\sum_{\sigma_{1}, \cdots, \sigma_{r} \in S_{N_{2}}} f\left(x_{1 \sigma_{1}(1)}, x_{2 \sigma_{2}(1)}, \cdots, x_{r \sigma_{r}(1)}\right) y_{1}\right. \\
& f\left(x_{1 \sigma_{1}(2)}, x_{2 \sigma_{2}(2)}, \cdots, x_{r \sigma_{r}(2)}\right) y_{2} \cdots y_{N_{2}-1} f\left(x_{1 \sigma_{1}\left(N_{2}\right)}, x_{2 \sigma_{2}\left(N_{2}\right)}, \cdots, x_{r \sigma_{r}\left(N_{2}\right)}\right) \\
& =0\}
\end{aligned}
$$

where $x_{i j}$ take values in $L^{s}$ and $y_{k}$ 's are arbitrary products of independent variables taking values in $L$. Thus values of $y_{k}$ 's lie in $A$.

Indeed, these weak identities are satisfied by the pair $(\widetilde{L}, \widetilde{A})$. It remains to notice that the pair $(L, A)$ and $(\widetilde{L}, \widetilde{A})$ satisfy the same multilinear weak identities.

Let $N$ be the minimal integer such that $(L, A)$ satisfies the weak identities $F_{N}=0$.

Let $a=F_{N-1}\left(a_{i j}, b_{k}\right) \neq 0, a_{i j} \in L^{s}, 1 \leq i \leq r, 1 \leq j \leq N-1 ; b_{k} \in A$. Choose $t>\max _{i, j} \operatorname{deg}\left(a_{i j}\right)$. By regularity of the polynomial $f$, there exist homogeneous elements $a_{1}, \cdots, a_{r} \in L^{t}$ such that $f\left(a_{1}, \cdots, a_{r}\right) \neq 0$. The identities $F_{N}=0$ immediately imply the following lemma.

Lemma 6.10. Let $b_{\mu}, c_{\mu} \in A+F 1$. Then $\left(\sum_{\mu} b_{\mu} f\left(a_{1}, \cdots, a_{r}\right) c_{\mu}\right) a$ is a linear combination of elements $\left(\sum_{\mu} b_{\mu} f\left(a_{1}^{\prime}, \cdots, a_{r}^{\prime}\right) c_{\mu}\right) a^{\prime}$, where $a^{\prime} \in$ $A, a_{1}^{\prime} \in\left\{a_{1}, a_{1 k}, 1 \leq k \leq N-1\right\}, a_{2}^{\prime} \in\left\{a_{2}, a_{2 k}, 1 \leq k \leq N-1\right\}, \cdots$, and at least one $a_{i}^{\prime}$ lies in $\left\{a_{i k}, 1 \leq k \leq N-1\right\}$.

The ideal $I$ generated by $f\left(a_{1}, \cdots, a_{r}\right)$ in $A$ has finite codimension. Let $A^{l} \subseteq I$.

Corollary 6.11. Let $u$ be a homogeneous element of degree $\geq l$. Then for arbitrary $b_{\mu}, c_{\mu} \in A+F 1$ the element $\left(\sum_{\mu} b_{\mu} u c_{\mu}\right) a$ is a linear combination of elements $\left(\sum_{\mu} b_{\mu} u^{\prime} c_{\mu}\right) a^{\prime}$, where $u^{\prime} \in A$ are homogeneous elements, $\operatorname{deg} u^{\prime}<\operatorname{deg} u$.

Indeed, from $u \in A^{\ell} \subseteq I$ it follows that $u=\sum_{\nu} b_{\nu}^{\prime} f\left(a_{1}, \cdots, a_{r}\right) c_{\nu}^{\prime}$, where $b_{\nu}^{\prime}, c_{\nu}^{\prime}$ are homogeneous elements, $\operatorname{deg} b_{\nu}^{\prime}+\operatorname{deg} c_{\nu}^{\prime}+\sum_{i=1}^{r} \operatorname{deg} a_{i}=$ 
$\operatorname{deg} u$. Lemma 6.10 implies that $\left(\sum_{\mu, \nu} b_{\mu} b_{\nu}^{\prime} f\left(a_{1}, \cdots, a_{r}\right) c_{\nu}^{\prime} c_{\mu}\right) a$ is a linear combination of elements $\left(\sum_{\mu, \nu}^{\mu} b_{\mu} b_{\nu}^{\prime} f\left(a_{1}^{\prime}, \cdots, a_{r}^{\prime}\right) c_{\nu}^{\prime} c_{\mu}\right) a^{\prime}$, where $\operatorname{deg} a_{i}^{\prime} \leq \operatorname{deg} a_{i}$ for all $i$ and at least for one $i$, we have $\operatorname{deg} a_{i}^{\prime}<\operatorname{deg} a_{i}$. Hence for $u^{\prime}=\sum_{\nu} b_{\nu}^{\prime} f\left(a_{1}^{\prime}, \cdots, a_{r}^{\prime}\right) c_{\nu}^{\prime}$ we have $\operatorname{deg} u^{\prime}<\operatorname{deg} u$.

Lemma 6.12. Let $h\left(x_{1}, \cdots, x_{q}\right)$ be a multilinear element of the free associative algebra such that for arbitrary homogeneous elements $u_{1}, \cdots, u_{q} \in A$ of degrees $\operatorname{deg} u_{1}<l, \cdots, \operatorname{deg} u_{q}<l$ we have $h\left(u_{1}, \cdots, u_{q}\right)=0$. Then $h=0$ holds identically on $A$.

Proof. Let $v_{1}, \cdots, v_{q} \in A$ be homogeneous elements of $A$ such that $h\left(v_{1}, \cdots, v_{q}\right) \neq 0$. Let the total degree $\sum_{i=1}^{q} \operatorname{deg}\left(v_{i}\right)$ be minimal among all $q$-tuples with this property. At least one element $v_{i}$ has degree $\geq l$.

Let us show that the graded just infinite algebra $A$ is graded prime. Indeed, if $I_{1}, I_{2}$ are nonzero graded ideals of $A$ then $A^{t_{1}} \subseteq I_{1}, A^{t_{2}} \subseteq$ $I_{2}$ for some integers $t_{1}, t_{2} \geq 1$. If $I_{1} I_{2}=(0)$ then $A^{t_{1}+t_{2}}=(0)$, a contradiction.

Hence there exists an element $b \in A$ such that $h\left(v_{1}, \cdots, v_{q}\right) b a \neq 0$.

By Corollary 6.11 the element $h\left(v_{1}, \cdots, v_{q}\right) b a$ is a linear combination of elements $h\left(v_{1}, \cdots, v_{i-1}, v^{\prime}, v_{i+1}, \cdots, v_{q}\right) b a^{\prime}$, where $\operatorname{deg} v^{\prime}<\operatorname{deg} v_{i}$. This contradicts the minimality of $\sum_{i=1}^{q} \operatorname{deg}\left(v_{i}\right)$ and finishes the proof of the lemma.

Remark. A nonzero element $h\left(x_{1}, \cdots, x_{q}\right)$ satisfying the hypothesis of Lemma 6.12 exists for an arbitrary finitely generated algebra. Moreover, for an arbitrary associative algebra $A$ and a finite dimensional subspace $V \subset A$, there exists a multilinear element $h\left(x_{1}, \cdots, x_{q}\right)$ of the free associative algebra such that $h\left(u_{1}, \cdots, u_{q}\right)=0$ for all elements $u_{1}, \cdots, u_{q} \in V$. Indeed, it is sufficient to choose an element that is skew-symmetric in $x_{1}, \cdots, x_{q}$, where $q=\operatorname{dim}_{F} V+1$, for example the element

$$
h\left(x_{1}, \cdots, x_{q}\right)=\sum_{\sigma \in S_{q}}(-1)^{|\sigma|} x_{\sigma(1)} \cdots x_{\sigma(q)} .
$$


Proof of Theorem 1.1. It is known (see $\mathrm{NvO}$ ) that a graded prime algebra is prime. By Lemma 6.12, $A$ is a PI-algebra. The prime PIalgebra $A$ has a nonzero center $Z$ and the ring of fractions $(Z \backslash\{0\})^{-1} A$ is a finite dimensional associative algebra over the field $(Z \backslash\{0\})^{-1} Z$ (see [Row]). Now the Engel-Jacobson theorem JJac2] implies that the algebra $A$ is nilpotent, a contradiction. Thus Theorem 1.1 is proved.

Proof of Theorem 1.2. Let $G$ be a residually- $p$ finitely generated torsion group. Let $G=G_{1} \geq G_{2} \geq \cdots$ be the Zassenhaus filtration. Consider the Lie algebra $L_{p}(\bar{G})=\bigoplus_{i \geq 1} G_{i} / G_{i+1}$. Because of the torsion property of elements of $G$, an arbitrary homogeneous element $a \in G_{i} / G_{i+1}$ of the Lie algebra $L_{p}(G)$ is ad-nilpotent (see [Kos3, VL]).

Consider the subalgebra $L$ of $L_{p}(G)$ generated by $G_{1} / G_{2}$. If the Lie algebra $L_{p}(G)$ satisfies a polynomial identity then by Theorem 1.1 the finitely generated Lie algebra $L$ is nilpotent. This implies that the pro$p$ completion $G_{\hat{p}}$ of the group $G$ is $p$-adic analytic and therefore linear (see DdSMS]). Now finiteness of $G$ follows from theorems of Burnside and Schur [Jac3].

Proof of Theorem 1.3. Let $F r$ be the free pro- $p$ group. Let $F r=F r_{1}>$ $\mathrm{Fr}_{2}>\cdots$ be the Zassenhaus filtration of $F r$. Suppose that the pro- $p$ completion $G_{\hat{p}}$ satisfies the pro- $p$ identity $w=1, w \in F r_{n} \backslash F r_{n+1}$, hence $w=\rho_{1}^{p^{s_{1}}} \cdots \rho_{r}^{p^{s_{r}}} w^{\prime}$, where each $\rho_{i}$ is a left normed group commutator of length $l_{i}, p^{s_{i}} \cdot l_{i}=n, w^{\prime} \in F r_{n+1}$.

Considering, if necessary, $\left[w, x_{0}\right]$ instead of $w$, we can assume that $n$ is not a multiple of $p$, and $w=\rho \cdots \rho_{r} w^{\prime}$, where all commutators $\rho_{1}, \cdots, \rho_{r}$ are of length $n$.

Let $\bar{\rho}_{i}$ be the commutator from the free Lie algebra that mimics the group commutator $\rho_{i}$. Then the Lie algebra $L_{p}(G)$ satisfies the polynomial identity $\sum_{i} \bar{\rho}_{i}=0$. By Theorem 1.2 we conclude that $|G|<$ $\infty$. 


\section{REFERENCES}

[Ami] S. A. Amitsur. Identities in rings with involutions. Israel J. Math., 7:63-68, 1969.

[Bah] Yu. A. Bahturin. Identical relations in Lie algebras. VNU Science Press, b.v., Utrecht, 1987. Translated from the Russian by Bahturin.

[DdSMS] J. D. Dixon, M. P. F. du Sautoy, A. Mann, and D. Segal. Analytic prop groups, volume 61 of Cambridge Studies in Advanced Mathematics. Cambridge University Press, Cambridge, second edition, 1999.

[FLGGL] Antonio Fernández López, Esther García, and Miguel Gómez Lozano. The Jordan algebras of a Lie algebra. J. Algebra, 308(1):164-177, 2007.

[Gol] E. S. Golod. On nil-algebras and finitely approximable p-groups. Izv. Akad. Nauk SSSR Ser. Mat., 28:273-276, 1964.

[Gri1] R. I. Grigorchuk. Degrees of growth of finitely generated groups and the theory of invariant means. Izv. Akad. Nauk SSSR Ser. Mat., 48(5):939985, 1984.

[Gri2] A. N. Grishkov. Local nilpotency of an ideal of a Lie algebra generated by second-order elements. Sibirsk. Mat. Zh., 23(1):181-182, 222, 1982.

[GS] Narain Gupta and Saïd Sidki. On the Burnside problem for periodic groups. Math. Z., 182(3):385-388, 1983.

[Jac1] Nathan Jacobson. Structure and representations of Jordan algebras. American Mathematical Society Colloquium Publications, Vol. XXXIX. American Mathematical Society, Providence, R.I., 1968.

[Jac2] Nathan Jacobson. Lie algebras. Dover Publications, Inc., New York, 1979. Republication of the 1962 original.

[Jac3] Nathan Jacobson. Basic algebra. II. W. H. Freeman and Co., San Francisco, Calif., 1980.

[Jac4] Nathan Jacobson. Structure theory of Jordan algebras, volume 5 of University of Arkansas Lecture Notes in Mathematics. University of Arkansas, Fayetteville, Ark., 1981.

[Kan] I. L. Kantor. Classification of irreducible transitive differential groups. Dokl. Akad. Nauk SSSR, 158:1271-1274, 1964.

[Kap] Irving Kaplansky. Rings with a polynomial identity. Bull. Amer. Math. Soc., 54:575-580, 1948.

[Koe] Max Koecher. Imbedding of Jordan algebras into Lie algebras. I. Amer. J. Math., 89:787-816, 1967.

[Kos1] A. I. Kostrikin. The Burnside problem. Izv. Akad. Nauk SSSR Ser. Mat., 23:3-34, 1959.

[Kos2] A. I. Kostrikin. Sandwiches in Lie algebras. Mat. Sb. (N.S.), 110(152)(1):3-12, 159, 1979.

[Kos3] A. I. Kostrikin. Around Burnside, volume 20 of Ergebnisse der Mathematik und ihrer Grenzgebiete (3) [Results in Mathematics and Related Areas (3)]. Springer-Verlag, Berlin, 1990. Translated from the Russian and with a preface by James Wiegold.

[Kur] A. Kurosch. Ringtheoretische Probleme, die mit dem Burnsideschen Problem über periodische Gruppen in Zusammenhang stehen. Bull. Acad. Sci. URSS. Sér. Math. [Izvestia Akad. Nauk SSSR], 5:233-240, 1941. 
[KZ] A. I. Kostrikin and E. I. Zel'manov. A theorem on sandwich algebras. Trudy Mat. Inst. Steklov., 183:106-111, 225, 1990. Translated in Proc. Steklov Inst. Math. 1991, no. 4, 121-126, Galois theory, rings, algebraic groups and their applications (Russian).

[Lev] Jakob Levitzki. On the structure of algebraic algebras and related rings. Trans. Amer. Math. Soc., 74:384-409, 1953.

[LS] T. H. Lenagan and Agata Smoktunowicz. An infinite dimensional affine nil algebra with finite Gelfand-Kirillov dimension. J. Amer. Math. Soc., 20(4):989-1001, 2007.

[Mar] Wallace S. Martindale, III. Prime rings satisfying a generalized polynomial identity. J. Algebra, 12:576-584, 1969.

[McC1] Kevin McCrimmon. A general theory of Jordan rings. Proc. Nat. Acad. Sci. U.S.A., 56:1072-1079, 1966.

[McC2] Kevin McCrimmon. A taste of Jordan algebras. Universitext. SpringerVerlag, New York, 2004.

[MZ] Kevin McCrimmon and Ephim Zel'manov. The structure of strongly prime quadratic Jordan algebras. Adv. in Math., 69(2):133-222, 1988.

[NvO] C. Năstăsescu and F. van Oystaeyen. Graded ring theory, volume 28 of North-Holland Mathematical Library. North-Holland Publishing Co., Amsterdam-New York, 1982.

[Row] Louis Rowen. Some results on the center of a ring with polynomial identity. Bull. Amer. Math. Soc., 79:219-223, 1973.

[Ser] Jean-Pierre Serre. Cohomologie galoisienne, volume 1965 of With a contribution by Jean-Louis Verdier. Lecture Notes in Mathematics, No. 5. Troisième édition. Springer-Verlag, Berlin-New York, 1965.

[Shi] A.I. Shirshov. On rings with identical relations, volume 43. Matem. Sbornic, 1957.

[The] Armin Thedy. $z$-closed ideals of quadratic Jordan algebras. Comm. Algebra, 13(12):2537-2565, 1985.

[Tit1] J. Tits. Une classe d'algèbres de Lie en relation avec les algèbres de Jordan. Nederl. Akad. Wetensch. Proc. Ser. A 65 = Indag. Math., 24:530-535, 1962 .

[Tit2] J. Tits. Algèbres alternatives, algèbres de Jordan et algèbres de Lie exceptionnelles. I. Construction. Nederl. Akad. Wetensch. Proc. Ser. A 69 = Indag. Math., 28:223-237, 1966.

[VL] Michael Vaughan-Lee. The restricted Burnside problem, volume 8 of London Mathematical Society Monographs. New Series. The Clarendon Press, Oxford University Press, New York, second edition, 1993.

[Zel1] E. I. Zel'manov. Primary Jordan algebras. Algebra i Logika, 18(2):162175, 253, 1979.

[Zel2] E. I. Zel'manov. Prime Jordan algebras. II. Sibirsk. Mat. Zh., 24(1):89104, 192, 1983.

[Zel3] E. I. Zel'manov. Characterization of the McCrimmon radical. Sibirsk. Mat. Zh., 25(5):190-192, 1984.

[Zel4] E. I. Zel'manov. Solution of the restricted Burnside problem for groups of odd exponent. Izv. Akad. Nauk SSSR Ser. Mat., 54(1):42-59, 221, 1990. 
[Zel5] E. I. Zel'manov. Solution of the restricted Burnside problem for 2groups. Mat. Sb., 182(4):568-592, 1991.

[Zel6] E. I. Zel'manov. On periodic compact groups. Israel J. Math., 77(12):83-95, 1992.

[Zel7] Efim Zelmanov. Nil rings and periodic groups. KMS Lecture Notes in Mathematics. Korean Mathematical Society, Seoul, 1992. With a preface by Jongsik Kim.

[Zel8] Efim I. Zelmanov. On the restricted Burnside problem. In Proceedings of the International Congress of Mathematicians, Vol. I, II (Kyoto, 1990), pages 395-402. Math. Soc. Japan, Tokyo, 1991.

[ZSSS] K. A. Zhevlakov, A. M. Slin'ko, I. P. Shestakov, and A. I. Shirshov. Rings that are nearly associative, volume 104 of Pure and Applied Mathematics. Academic Press, Inc. [Harcourt Brace Jovanovich, Publishers], New York-London, 1982. Translated from the Russian by Harry F. Smith.

Efim Zelmanov, Ezelmano@math.ucsd.edu, Department of Mathematics, University of California, San Diego, 9500 Gilman Drive, La Jolla, CA 92093-0112, U.S.A. 